\title{
Acoustic concerns related to multi cultural societies
}

\section{Gade, Anders Christian}

\section{Published in:}

Acoustical Society of America. Journal

Publication date:

2001

\section{Document Version}

Publisher's PDF, also known as Version of record

Link back to DTU Orbit

Citation (APA):

Gade, A. C. (2001). Acoustic concerns related to multi cultural societies. Acoustical Society of America. Journal, 110(5), 2664-2664.

\section{General rights}

Copyright and moral rights for the publications made accessible in the public portal are retained by the authors and/or other copyright owners and it is a condition of accessing publications that users recognise and abide by the legal requirements associated with these rights.

- Users may download and print one copy of any publication from the public portal for the purpose of private study or research.

- You may not further distribute the material or use it for any profit-making activity or commercial gain

- You may freely distribute the URL identifying the publication in the public portal

If you believe that this document breaches copyright please contact us providing details, and we will remove access to the work immediately and investigate your claim 


\title{
Session $2 \mathrm{aAB}$
}

\section{Animal Bioacoustics: Fish Audition and Sound Production}

\author{
Mardi C. Hastings, Chair \\ Biomedical Engineering Center, The Ohio State University, 1080 Carmack Road, Columbus, Ohio 43210
}

Chair's Introduction-8:00

Invited Papers

\begin{abstract}
8:05
2aAB1. The enigma of fish ear diversity. Arthur N. Popper, Xiaohong Deng, John Ramcharitar, and Dennis M. Higgs (Dept. of Biol. and Neurosci. and Cognit. Sci. Prog., Univ. of Maryland, College Park, MD 20742, ap17@umail.umd.edu)

There are substantial interspecific differences in the gross anatomy of fish inner ears, including the relative size of the different end organs and the sizes and shapes of their otoliths and sensory epithelia. Differences also occur at finer structural levels and include the orientation patterns of the ciliary bundles on the sensory hair cells. There is also substantial structural variation along the lengths of a single sensory epithelium. This includes not only the mode of contact between the sensory epithelium and the otolith, but also the lengths of the ciliary bundles on the hair cells, and the ultrastructure of the hair cell bodies themselves. The functional significance of the interspecific differences in ear structure is far from being understood, but it may reflect the evolution of different ways to do the same basic kinds of peripheral signal processing, or different kinds of signal processing. We also do not understand the functional importance of the intraepithelial differences, although they may suggest variance in signal processing along the lengths of single epithelia. This paper will consider all of these differences, and suggest ways in which we might think about their functional significance.
\end{abstract}

8:30

2aAB2. Perception of sound by goldfish. Richard R. Fay (Parmly Hearing Inst., Loyola Univ. of Chicago, 6525 N. Sheridan Rd., Chicago, IL 60626)

Psychophysical experiments have helped define the sense of hearing of goldfish with respect to detection and discrimination thresholds within many acoustic dimensions. Generally, these data reveal aspects of peripheral and early brain processing that are qualitatively, and sometimes quantitatively, indistinguishable from those of most other vertebrates. This correspondence suggests that goldfish share an essentially common sense of hearing with other vertebrates, and motivated new experiments that investigate more complex aspects of sound perception in goldfish using the phenomenon of stimulus generalization to study perceived similarities among simple and complex sounds. We have found that goldfish listen analytically to simultaneous tones, have perceptual dimensions analogous to pitch, timbre, and roughness in human listeners, and can segregate simultaneous, complex sources in perception. Several of these processes will be illustrated. These results are in accord with the hypothesis that the sense of hearing in goldfish is essentially similar to that revealed in other vertebrates studied. We suggest that it is useful to view the sense of hearing of any given vertebrate species as representing a general vertebrate pattern as an alternative to assuming that individual species are specially adapted to process particular sounds in its species-specific niche. [Work supported by NIH, NIDCD.]

8:55

2aAB3. Florida red tides and hearing. Zhongmin Lu and Zemin Xu (Dept. of Biol., Univ. of Miami, 1301 Memorial Dr., Rm. 4, Coral Gables, FL 33146, zlu@miami.edu)

Red tides, characterized by patches of colored seawater, have been reported worldwide. They are formed due to blooms of marine phytoplankton populations. Particularly, the dinoflagellate (Ptychodiscus-brevis) has been identified to cause the red tides occurring along the coastline of west Florida. It is known that $P$. brevis produces many types of neurotoxins, which result in massive kills of fishes, birds, and marine mammals including endangered species. Although some studies demonstrated effects of the neurotoxins on the respiratory system, heart, and neuromuscular junction, no study has ever been reported regarding possible effects of any red tide toxins on sensory systems. This study was to investigate whether or not a neurotoxin purified from Florida red tides, brevetoxin-3, affects hearing in fish. The neurotoxin was intraperitoneally injected into the goldfish (Carassiusauratus), and hearing thresholds were measured using auditory brainstem recording techniques. Results show that the toxin causes significant hearing loss in the goldfish. Species differences in neurotoxin tolerance and mechanisms of the hearing loss are discussed. Results from this study enhance our awareness of neurotoxin effects on sensory systems in vertebrates including humans. [Work supported by NIH Grant No. R29DC03275, UM start-up funds, and NIEHS pilot project award.] 
2aAB4. Listening in the dark: Behavioral and neural mechanisms of acoustic recognition in a singing fish. Andrew H. Bass (Dept. of Neurobiology \& Behavior, Cornell Univ., Ithaca, NY 14853)

Vocalizing species of teleost fish have a simple repertoire of species-typical signals central to their social and reproductive behavior. Male midshipman fish ("the California singing fish") acoustically court females using "hums" that are long duration (secs 1h) and spectrally simple. Individual hums exhibit an essentially flat envelope consisting of a low fundamental frequency $(90-100 \mathrm{~Hz})$ and several harmonics. By contrast, naturally occurring acoustic beats show envelope modulations: the hums of neighboring males overlap to produce a beat with a difference frequency $(0-8 \mathrm{~Hz})$ in which amplitude and phase modulations result from the continual shifting of the two fundamental frequencies. Behavioral playback (phonotaxis) studies show that individual midshipman can use multiple signal parameters including hum duration, gap duration between successive hums, fundamental frequency, and intensity to distinguish hums from each other, while the effectiveness of beats as playback signals depends upon both beat modulation rates and depth of modulation. Neurophysiological evidence shows that auditory neurons in the midshipmans VIIIth nerve and midbrain encode the physical attributes of these signals mainly using spike rate and spike synchronization, mechanisms that we propose contribute to the neural templates adapted for both hum and beat recognition. [Research supported by NIH.]

$$
\text { 9:45-10:05 Break }
$$

\section{0:05}

2aAB5. Using passive acoustics to monitor spawning of fishes in the drum family (Sciaenidae). Joseph Luczkovich (Inst. for Coastal and Marine Resources and Dept. of Biol., East Carolina Univ., Greenville, NC 27858) and Mark Sprague (Dept. of Phys., East Carolina Univ., Greenville, NC 27858)

The goal of this study was to develop a passive acoustic survey protocol to identify spawning habitats of sciaenid fishes. Based on comparisons of recordings of captive-fish and field recordings of species-specific courtship sounds, spawning areas of red drum, weakfish, spotted sea trout, and silver perch (Family Sciaenidae) have been identified in Pamlico Sound, NC. Sciaenid sounds were recorded using either a portable hydrophone deployed from a boat or timer-operated sonobuoys. Loudness of mating choruses of weakfish and silver perch was strongly correlated with the abundance of pelagic sciaenid-type eggs, suggesting that these areas were used for spawning. Sonobuoy recordings showed that weakfish spawned only in the high-salinity habitats near the inlets, spotted sea trout spawned predominantly in the low-salinity areas near the river mouths, silver perch spawned in both high- and low-salinity areas, and red drum spawned most commonly in low-salinity areas, but only in September. The loudness of mating choruses of silver perch was diminished when vocalizing bottlenose dolphins were present in the area around sonobuoys or when recordings of signature whistles were played back. Such "acoustical avoidance" behavior causes variation in sound pressure levels and could lead to lower estimates of abundance of spawning adults when conducting passive acoustic surveys.

\section{0:30}

2aAB6. Chorus behavior ecology in warm temperature sciaenid (Pisces: Sciaenidae) within a warm-temperate to tropical coastal lagoon. R. Grant Gilmore, Jr. (Dynamac Corp., Mail Code DYN-8, Kennedy Space Ctr., FL 32899, rggilmorej@aol.com)

Individual sound production and aggregative choral behaviors in sciaenid fishes of the Indian River Lagoon system of east central Florida have been under study since 1977. Long term studies of sonifery augmented with equally long term life history research has allowed classification of their call behaviors relative to their ecology. Five sciaenid species, silver perch, Bairdiella chrysoura, spotted seatrout, Cynoscion nebulosus, black drum, Pogonias cromis, red drum, Sciaenops ocellata, and weakfish, Cynoscion regalis can be divided into temporal, spatial, morphological, and vocal guilds. These guilds reveal partitioning of estuarine resources not only for habitat, food, and predation avoidance, but also for reproductive biology, sonifery, and larval-juvenile ecology. Variation in call type is significant between these species further presenting implications for co-evolved mating strategies under syntopic conditions within confined lagoonal ecosystems.

\section{Contributed Papers}

\section{0:55}

2aAB7. Pulsed sound production by Sciaenid fishes. Mark W. Sprague (Dept. of Phys., East Carolina Univ., Greenville, NC 27858, spraguem@mail.ecu.edu)

Many fishes, including members of the family Sciaenidae, produce pulses of sound by driving their swimbladders with sonic muscles. The swimbladder functions as a damped oscillator driven by the sonic muscles in a transient response. The radiated waveform contains information about the excitation function generated by the sonic muscles and the properties of the swimbladder. A theoretical model for pulsed sound production based on a driven damped oscillator [M. W. Sprague, J. Acoust. Soc. Am. 108, 2430-2437 (2000)] can be used to establish relationships between sonic muscle excitation, swimbladder motion, and the radiated waveform. Analysis of sonic muscle excitations and corresponding waveforms produced by weakfish (Cynoscion regalis) (Sciaenidae) suggest that the swimbladder continues to oscillate or "ring" after the sonic muscle driv- ing force stops. Portions of the waveform produced during this ringing of the swimbladder depend solely on swimbladder properties such as volume, which relates to fish size, and damping.

\section{1:10}

2aAB8. Psychoacoustics of ultrasound detection in clupeids. David A. Mann (College of Marine Sci., Univ. of South Florida, 140 7th Ave. S., St. Petersburg, FL 33701 and Mote Marine Lab., 1600 Ken Thompson Pkwy., Sarasota, FL 34236, dmann@marine.usf.edu), William N. Tavolga (Mote Marine Lab., 1600 Ken Thompson Pkwy., Sarasota, FL 34236), Dennis M. Higgs, and Arthur N. Popper (Dept. of Biol., Univ. of Maryland, College Park, MD 20742)

Recent behavioral and physiological studies have shown that some clupeid fishes are able to detect ultrasonic signals up to $180 \mathrm{kHz}$. Fishes in the subfamily Alosinae, including the American shad (Alosa sapidissima) and the gulf menhaden (Brevoortia patronus) are able to detect ultrasound. Yet other clupeids, including the scaled sardine (Harengula jaguana), Spanish sardine (Sardinella aurita), and the bay anchovy (Anchoa mitch- 
illi), are not able to detect ultrasound. In this paper we review the ability of clupeids to detect ultrasound, and investigate the psychoacoustics of ultrasound detection, including frequency discrimination and masking by lowfrequency and high-frequency sounds. [Work supported by NSF and NIH.]

\section{$11: 25$}

2aAB9. Effect of swimbladder resonance on auditory bandwidth and sensitivity in teleost fishes. Mardi C. Hastings, Corrie J. Derenburger (Biomed. Eng. Ctr. and Dept. of Mech. Eng., Ohio State Univ., 1080 Carmack Rd., Columbus, OH 43210, hastings.6@osu.edu), and James J. Finneran (Sci. Applications Intl. Corp., Maritime Services Div., 3990 Old Town Ave., Ste. 105A, San Diego, CA 92110)

Experimental studies of four teleost species indicate that the response of the swimbladder to sound controls the auditory bandwidth of fish both with and without a direct connection between the swimbladder and the inner ear. Motion of the swimbladder in an acoustic field generated by an underwater loudspeaker was measured in vivo from 200 to $3000 \mathrm{~Hz}$ for channel catfish (Ictalurus punctatus), goldfish (Carassius auratus), American shad (Alosa sapidissima), and oscars (Astronotus ocellatus). Data were normalized with local acoustic energy density to obtain swimbladder frequency responses. Channel catfish and goldfish have two swimbladder chambers and Weberian ossicles that connect the anterior chamber to the inner ear, while American shad and oscars have a single swimbladder and no Weberian osscicles. (Shad do have auditory bullas; however, no motion of the bullas was detected.) Swimbladder frequency responses were compared with audiograms reported in the literature. Results indicate that auditory bandwidth correlates directly with the bandwidth of swimbladder resonance in all four species. Auditory threshold, however, does not correlate with amplitude of the swimbladder resonance. The two species with Weberian ossicles have lower auditory thresholds, but swimbladder resonances of approximately the same or somewhat lower amplitude than the other two species.

\title{
Session $2 \mathrm{aAO}$
}

\section{Acoustical Oceanography and Physical Acoustics: Turbulence and Finestructure Studies I: Water Column Turbulence}

\author{
Alex E. Hay, Chair \\ Department of Oceanography, Dalhousie University, Halifax, Nova Scotia B3H 4J1, Canada
}

Chair's Introduction-8:00

Invited Papers

8:05

2aAO1. Small-scale processes and vertical diffusion in the ocean. Ann E. Gargett (Ctr. for Coastal Physical Oceanogr., Old Dominion Univ., Norfolk, VA 23529, gargett@ccpo.odu.edu)

A depth profile taken anywhere in the ocean reveals large vertical gradients of oceanic water properties such as temperature, salinity, oxygen concentration, macronutrients such as nitrate and silicate, and micronutrients such as iron. These vertical gradients are formed at the very largest (depth/basin) scales of motion in the sea, and eradicated at the very smallest scales, those associated with three-dimensional turbulence. Turbulent enhancement of vertical diffusion over that associated with molecular motions is central to vital processes within the ocean. Vertical diffusion resupplies nutrients (the "fertilizer" for marine plant life) to the upper sunlit layers of the sea, underpinning marine food webs. The location and strength of turbulent diffusion determines the distribution of manmade pollutants introduced into the ocean, whether by design or by accident. More surprising perhaps is the essential role played by vertical diffusion in determining large-scale oceanic density structure, hence the crucial importance of small-scale turbulent processes to studies of natural and/or anthropogenic climate change. This presentation will review a variety of processes known or believed to generate the smallest scales of velocity and scalar variance in the sea, and describe the tools oceanographers presently use to measure and interprete salient characteristics of these small-scale processes.

9:05

2aAO2. The measurement of flow and turbulence levels using acoustical scintillation analysis. Daniela Di Iorio (Univ. of Georgia, 220 Marine Sci. Bldg., Athens, GA 30602)

For acoustical propagation in the coastal environment, small scale fluctuations in the temperature/salinity and flow velocity distorts the spherically spreading phase fronts which result in amplitude and phase fluctuations (scintillation). As the fine structure variability in the medium has a natural coherence time, it will be transported by the current. Detection of pattern motion is an essential concept exploited in acoustical scintillation and has been demonstrated in a variety of coastal channels. Important features of this approach are that the measurement is path averaged, is of the flow component perpendicular to the acoustic path and is sensitive to the Fresnel scale size which is chosen to lie within the inertial subrange of the turbulent random medium. Analysis of the signals goes beyond measurement of flow speed, and has included detection of turbulent velocity and scalar levels and turbulent anisotropy. For one-way propagation the turbulent kinetic energy dissipation rate is derived from the variance of the log-amplitude signal which is dependent on the level of the Kolmogorov spectrum dominated by current fluctuations. From reciprocal propagation, scalar and velocity turbulence levels are derived from accurate phase fluctuations. Detection of turbulent anisotropy is based on horizontal and vertical angle of arrival correlations using a square array with $1 \mathrm{~m}$ sides and thus sensitive to turbulent scale sizes approximately a few meters. 
2aA03. Acoustic measurements of global ocean shear. Robert Pinkel, Luc Rainville, Eric Slater, Lloyd Green, Mike Goldin, and Mai Bui (Scripps Inst. of Oceanogr., La Jolla, CA 92093-0213)

The energetics of the ocean can be viewed as a cascade, with energy input at relatively large spatial scales, 1-10 $000 \mathrm{~km}$, and eventually dissipated by turbulent processes at centimeter-millimeter scales. The turbulence is thought to be triggered by the small-scale shear in the sea. Since the shear field serves as a mediator of ocean mixing, its dynamics are of central relevance to the overall mechanics of the ocean. To gather global shear data, a dual frequency Hydrographic Doppler Sonar System has been developed for the Scripps research vessel Roger Revelle. The System consists of a nested pair of Janus transducers operating at 50 and $140 \mathrm{kHz}$. The $50 \mathrm{kHz}$ sonar profiles to depths of 700-1000 m with $15 \mathrm{~m}$ depth resolution. The $140 \mathrm{kHz}$ sonar achieves ranges of 200-300 m with $3 \mathrm{~m}$ depth resolution. Operating since January 2000, the system has detected considerable geographic variability in both shear and acoustic scattering strength. The deep Sea of Japan and the Eastern North Pacific are surprisingly low in shear. Large shears are encountered near the Hawaiian Ridge and in the waters beneath the Kuroshio. With continued operation, the large-scale patterns and underlying governing principles of the shear field will hopefully emerge.

\section{9:55}

2aAO4. Acoustic measurement of oceanic turbulent kinetic energy dissipation rate, using a large-eddy technique. Ann E. Gargett (Ctr. for Coastal Physical Oceanogr., Old Dominion Univ., Norfolk, VA 23529, gargett@ ccpo.odu.edu)

Turbulence in the ocean results from many different processes operating over a wide range of time and space scales, with spatial and temporal variability particularly extreme in coastal oceans. If origins and effects of turbulent processes in the ocean are to be understood, it is essential to supplement the small number of accurate measurements of turbulent properties produced by current microprofiler techniques. This talk will discuss a new acoustic large-eddy technique for estimating turbulent kinetic energy dissipation rate $\epsilon$, using measurements of vertical velocity from a specialized Acoustic Doppler Current Profiler (ADCP). The method was calibrated against a subset of $\epsilon$ profiles taken during a single cruise with now-standard airfoil probes carried on a free-fall microprofiler. Without further adjustment of the calibration constant, the large-eddy acoustic estimates successfully describe the remaining profiler data from the same cruise, as well as data obtained with a different microscale profiler on a subsequent cruise. The new technique reveals the complexity of the field of $\epsilon$ in energetic coastal tidal fronts, on spatial and temporal scales which cannot be resolved by standard microprofiler measurements.

\section{0:20-10:35 Break}

\section{Contributed Papers}

\section{0:35}

2aAO5. Observation of small scale fluid processes in the vicinity of continental shelf breaks using high-frequency acoustic backscattering systems. Marshall H. Orr (The Naval Res. Lab., 4555 Overlook Ave. S.W., Washington, DC 20375)

Acoustic signal vertical and horizontal coherence properties are controlled on the shelf and near the shelf break by a variety of flow induced fluid processes. These processes perturb the sound speed field at spatial scales of a few hundred meters and less. The frequency of occurrence, depth distribution, and the relationship of the sound speed field variability to both oceanic and atmospheric fluid processes occurring on the continental shelf is poorly understood and are not generally included in most acoustic propagation models. Acoustic flow visualization records collected on the continental shelf and at the shelf break in water depths ranging from 50-300 m will be presented. These records will show: (1) the types of internal wave and smaller scale turbulence that occur in the summer and early fall in the vicinity of the New Jersey shelf; (2) some observation of the dissipation of large amplitude internal waves at the shelf break of the South China Sea; and (3) a variety of small scale instabilities that can perturb the sound speed field. [Work supported by the ONR Ocean Acoustic Program and ONRs NRL base funding.]

\section{0:50}

2aAO6. Laboratory measurements of acoustic scattering from a temperature and salinity gradient. Joseph D. Warren, ${ }^{\text {a) Andone C. }}$ Lavery, Ray W. Schmitt, and Timothy K. Stanton (Woods Hole Oceanogr. Inst., Woods Hole, MA 02543, jwarren@whoi.edu)

Recently developed theoretical scattering models predict that acoustic scattering from temperature and salinity microstructure at high frequencies $(10 \mathrm{kHz}-1 \mathrm{MHz})$ can be significant under certain oceanographic conditions. The results of this theoretical work suggest that it may be possible to use acoustic scattering techniques, in combination with the scattering models, to estimate oceanographic parameters, such as the dissipation rate of turbulent kinetic energy. In addition, quantification of the scattering contributions from temperature and salinity microstructure can play an important role in correctly interpreting acoustic surveys of marine life, since recent field data indicate that the acoustic returns from zooplankton and microstructure can be of similar strength. Acoustic backscatter from a sharp temperature and salinity gradient was measured in a tank capable of generating, and sustaining, a thin double-diffusive layer $(1-2 \mathrm{~cm}$ thick) between two water masses (cold, fresh residing above warm, salty water). Vertical shear, temperature, and salinity profiles were measured during the experiment to provide input to the acoustic scattering models. Backscatter was measured at frequencies between $24 \mathrm{kHz}$ and $500 \mathrm{kHz}$, and as a function of range from the sharp interface as part of a program to measure the backscattering from microstructure. ${ }^{\text {a) }}$ Currently at Southwest Fisheries Science Center, La Jolla, CA 92037.

\section{1:05}

2aA07. Acoustic scatter from thermal plumes. John Oeschger (Coastal Systems Station, 6703 W. Hwy. 98, Panama City, FL 32407)

Laboratory results of high-frequency acoustic scattering measurements from a thermally driven plume are reported. Farfield weak scattering theory describes the acoustic scatter from the medium variability. To first order, the dominant scattering mechanism is thermally driven sound speed variations that are related to temperature deviations from ambient. As a result, the received complex acoustic scatter is a measure of the onecomponent three-dimensional Fourier transform of the temperature difference field measured at the Bragg wave number; this is the Fourier component of the plume variability that produces scattering. Results are presented for multistatic scattering from unstable and turbulent plumes using a parallel scattering geometry. Through common Bragg wave number comparisons, the data justify the application of the farfield weak scattering theory to the present case of a thermal plume. Results of temperature time series measurements taken of the plumes are also shown. Acoustical and mechanical temperature spectral estimates are presented and show a near-classical $-\frac{11}{3}$ power law behavior. 
mately the same age could be assessed, and ran under different age sec-

2aAO8. Measured turbulence in ship wakes. R. Lee Culver and David Bradley (Grad. Prog. in Acoust. and Appl. Res. Lab., The Penn State Univ., P.O. Box 30, State College, PA 16804)

Large surface ships moving at high speed generate extremely turbulent velocity fields containing high concentrations of air bubbles that are distributed in a nonuniform manner. The bubbles are acoustically active, making sonar a useful tool for imaging the ship wake and measuring the spatial and temporal variations of the bubbles. We have employed a 250$\mathrm{kHz}$ multibeam sonar, integrated into an autonomous vehicle (AUV), to image the wakes of two large surface ships from the underside. The AUVs traveled at high speed so that the spatial variation of wake of approxi- tions of the wake in order to observe temporal variation. Using an assumed bubble size spectrum for the wake, bubble density has been estimated from the backscattered signals. Variations in bubble density with depth, cross-wake, and along-wake dimension, and with time, are observed. Spatial wave number spectra are used to quantify the scales over which bubbles are distributed, which may be related to the scales of turbulent energy distribution. Comparing measurements of different age wakes indicate how the distribution over spatial scales evolves with time. The multibeam sonar appears to provide quantitative information about the distribution of bubbles, and possibly turbulence, in the ship wake. [Work supported by ONR.]

11:35-12:00

Panel Discussion

TUESDAY MORNING, 4 DECEMBER 2001

ROOM 223, 8:00 A.M. TO 12:30 P.M.

Session 2aBB

\title{
Biomedical Ultrasound/Bioresponse to Vibration: Topical Meeting on Physics of Ultrasound in Relation to the Biology of its Therapeutic Effects: HIFU, LOFU, and Imaging HIFU Lesions
}

\author{
Pierre D. Mourad, Chair \\ Applied Physics Laboratory, University of Washington, 1013 NE 40th Street, Seattle, Washington 98105
}

Chair's Introduction-8:00

Invited Papers

8:05

2aBB1. MRI guided focused ultrasound opening of blood brain barrier. K. Hynynen, N. McDannold, N. Vykhodtseva, N. Sheikov, and F. Jolesz (Dept. of Radiol., Brigham and Women's Hospital and Harvard Med. School, 75 Francis St., Boston, MA 02115)

Previously, we demonstrated that focused ultrasound can focally open the blood brain barrier (BBB) reversibly without damage to the neurons. In this study, we varied the ultrasound pulse parameters to investigate their influence on BBB opening and any resulting tissue damage. Under magnetic resonance imaging (MRI) guidance, a series of targeted ultrasound bursts (1.5 MHz, burst duration: 5 cycles to $100 \mathrm{~ms}$, repetition frequency: $1 \mathrm{~Hz}-1 \mathrm{kHz}$ ) were delivered into rabbit brains at low pressure levels (up to approximately $5 \mathrm{MPa}$ ) after intravenous administration of a commercially available ultrasound contrast agent containing preformed gas bubbles. Focal MRI signal intensity changes were observed during the deposition of acoustic energy. BBB opening at the correct target was confirmed by detecting the localized uptake of an MRI contrast agent. In some animals Trypan Blue was injected intravenously after the sonications. The animals were sacrificed at time intervals ranging from $2 \mathrm{~h}$ to 7 days. Light and electron microscopy evaluation was performed. No neuronal damage was observed at the lowest power levels that produced BBB opening. No neuronal damage was observed at the lowest power levels that produced BBB opening. Correlation of focal MRI signal changes to the uptake of a large molecular size contrast agent was observed demonstrating the potential of image guided drug delivery.

$8: 25$

2aBB2. Dual-mode HIFU phased arrays for noninvasive surgery. Emad Ebbini (Dept. of Elec. and Computational Eng., Univ. of Minnesota, Minneapolis, MN 55455)

Ultrasound phased arrays employing advanced piezocomposite transducer technology are now commonplace in high-power therapeutic applications. When properly designed, these arrays can produce (single or multiple) therapeutic foci suitable for noninvasive surgery for a variety of applications. A properly designed HIFU array has a characteristic therapeutic operating field (ThxOF) around its geometric center. ThxOF defines the volume within which the array is capable of producing therapeutic effects at the intended focal spot(s) without collateral damage at other locations. Recently, we were able to show that HIFU arrays optimized for therapy has a characteristic imaging field of view (IxFOV) around its geometric center. IxFOV defines the volume within which the array is capable of producing images of the target at a specified contrast level. In this talk we outline the optimization procedure for designing dual-mode HIFU arrays suitable for imaging and therapy. In addition, we give illustrative experimental examples of dual-mode operation of HIFU phased arrays. In particular, we emphasize new imaging techniques for visualization of HIFU lesions, both discrete and volumetric. Images of HIFU lesions obtained by the dual-mode HIFU array and a commercial scanner will be compared to demonstrate the superiority of our specialized imaging modes in lesion visualization. 
2aBB3. Bubbles and acoustic image-guided high intensity focused ultrasound. Michael R. Bailey, Shahram Vaezy, Jonathan C. Yuen, Ajay Anand, Nathan A. Miller, Peter J. Kaczkowski, and Lawrence A. Crum (Ctr. for Industrial and Medical Ultrasound, APL/UW, 1013 NE 40th St., Seattle, WA 98105)

Clinical diagnostic ultrasound (US) can be used to target and to monitor in real-time high-intensity focused ultrasound (HIFU) therapy. In our system, the HIFU transducer (3.5 MHz, $35 \mathrm{~mm}$ aperture, $55 \mathrm{~mm}$ radius of curvature) and US scan head (several were tested, center frequencies $3-8 \mathrm{MHz}$ ) are fixed with the HIFU focus in the imaging plane. HIFU and US are either synchronized real time to relegate interference to the image fringe or HIFU and US are interlaced for nearly real-time imaging. HIFU produces a localized hyperechoic region visible on B-mode US. Coagulatively necrosed lesions produced have similar size, shape, and location to measurements made from the corresponding US images. Thresholds are also comparable. However, in vivo, if HIFU is turned off as soon as hyperecho appears, no lesion is seen (the tissue was fixed within four hours of treatment). Thus, a short HIFU burst can be used to target treatment. Bubbles appear to be largely but perhaps not entirely responsible for the increase in echogenicity. Times for dissipation of the hyperecho and dissolution of a bubble as a function of hydrostatic pressure compare well. Significant overpressure (50 bar) can suppress hyperecho produced by HIFU. [Work supported by NSF, NSBRI, DARPA/ONR, and NIH SBIR.]

\section{9:05}

2aBB4. High intensity focused ultrasound for arrest of bleeding. Shahram Vaezy, Roy Martin, and Lawrence Crum (Ctr. for Industrial and Medical Ultrasound, Appl. Phys. Lab., Univ. of Washington, Seattle, WA 98195)

High Intensity Focused Ultrasound (HIFU) has been shown to provide an effective method of hemostasis, in animal studies, for both solid organs and blood vessels (acoustic hemostasis). Moderate to profuse bleeding from major blood vessels, liver, and spleen, can be stopped within 1-2 min of HIFU application. The efficacy of acoustic hemostasis has been demonstrated using HIFU frequencies of 1-10 MHz, and acoustic intensities of 1000-5000 W/ $\mathrm{cm}^{2}$. Conventional B-mode ultrasound imaging and Doppler appear to provide a effective visualization, targeting, and monitoring methods when extracorporeal application, in case of internal bleeding, is desired. Both thermal and mechanical mechanisms of HIFU appear to be responsible for achieving hemostasis. The thermal mechanism, due to ultrasound absorption, raises the tissue temperature in excess of $70{ }^{\circ} \mathrm{C}$ in less than a second, leading to shrinkage of the tissue and collapse of small blood vessels. The mechanical mechanism, due to cavitation activity, disrupts the tissue structure, leading to the occlusion of large blood vessels. Both mechanisms result in coagulative necrosis of tissue, and arrest of bleeding. Acoustic hemostasis may provide an effective method in surgery and prehospital settings for treating bleeding in trauma and elective surgery patients.

\section{9:25}

2aBB5. On the increase of disintegration efficiency of an acoustic lithotripter. V. R. Singh (Dept. of Biomed. Measurements and Standards, Natl. Physical Lab., New Delhi-110012, India, vrs@csnpl.ren.nic.in)

Currently, disintegration of renal calculi, noninvasively, with the use of lithotripters, is in common use. In the hospitals, commercial lithotripters like extra-corporeal shock wave lithotripters (ESWL) are in routine use. These systems are very cumbersome and expensive. Treatment of the patients also takes comparatively more time, due to more number of sittings. The high ultrasonic intensity used in such systems has side effects, and causes damage to delicate nerves and fibers in the surroundings of the stones present in the kidney. Therefore, the present lithotripters are required to be improved by increasing the efficiency of disintegration and other factors. In the present work, the increase in the disintegration efficiency of the ultrasonic lithotripters, in this case Siemens ESWL, is made by stimulating externally the main shock wave with an acoustic stress wave. The cavitation bubbles are found to implode faster, with more disintegration efficiency of the lithotripter, giving in turn, better treatment to the patient, with less sitting time and less ultrasound dosage level.

\section{9:45-9:55 Break}

\section{9:55}

2aBB6. High intensity focused ultrasound treatment of abdominal tumors. G. ter Haar, I. Rivens, and M. Allen (Dept. of Phys., Inst. of Cancer Res., Royal Marsden Hospital, Sutton, Surrey SM2 5PT, UK)

A phase II clinical trial for the treatment of isolated liver metastases is currently underway at the Royal Marsden Hospital. Tumors lying 4-12 cm below the skin surface are targeted in fully conscious patients. Treatment success is monitored using either radiological methods in conjunction with biochemical marker assays, or by histological examination. Factors determining pain or other sensations have been studied in order that exposures may be optimized. Laboratory investigations have been undertaken in parallel with these clinical studies, and these have been used to establish the effects of, for example, rib shadowing, timing of individual exposures, and in situ intensity on tissue ablation. Skin studies have allowed the determination of thresholds for a "burn" when multiple, overlapping exposures are used.

\section{0:15}

2aBB7. Transrectal focused ultrasound treatment of localized prostate cancer. Jean-Yves Chapelon, RTmi Souchon, Laura Curiel, and Albert Gelet (Inserm U556, 151 cours A. Thomas, 69424 Lyon, France)

Since 1989, a research project with the aim of establishing treatment of localized prostate cancer by means of high intensity focused ultrasound (HIFU) has been developed. The treatment of localized prostate cancer with HIFU, is administered transrectally, using ultrasound guidance only. The quality of the induced necrosis depends to a large extent on the choice of firing parameters: power, frequency, numbers and position of shots, shots duration, waiting time between shots and the shape of the acoustical field. The 
lesions are created by a thermal effect. Their slightly conical form is due to the absorption of the ultrasound wave by the tissues, increased by cavitation bubbles. Recently, it has been shown that real time elastography has the potential to accurately depict the ablated volume during a HIFU session, therefore providing feedback on the treatment. Results obtained since 1993 demonstrate that transrectal HIFU prostate ablation is an effective therapeutic alternative for patients with localized prostatic adenocarcinoma.

\section{$10: 35$}

2aBB8. Ultrasonic monitoring of radiation-force deformation induced by therapeutic ultrasound. Frederic L. Lizzi, Robert Muratore, Cheri X. Deng, Samuel Mikaelian, S. Kaisar Alam, and Jeffrey A. Ketterling (Riverside Res. Inst., 156 William St., New York, NY 10038-2609)

We have developed a dual-transducer technique to monitor tissue deformation induced by therapeutic ultrasound. The technique is designed to monitor and control therapeutic lesion size and location. Currently, the technique employs a spherical-cap high-intensity transducer with a central, co-linear diagnostic transducer. A-mode rf echo signals are first acquired using the diagnostic transducer to document pre-exposure conditions within the target tissue. A short $(10 \mathrm{~ms})$ intense exposure is then delivered with the spherical-cap transducer in order to induce tissue motion by radiation force. A-mode rf signals are then acquired for $0.2 \mathrm{~s}$. Pre- and post-exposure A-mode signals are analyzed using correlation processing to determine the extent of induced tissue motion. The time constant associated with return to initial tissue positions is also calculated. These procedures have been applied in vitro to liver specimens using the spherical-cap transducer to periodically induce motion and to produce thermal lesions; at relatively low exposure levels, results are consistent with computer simulations. Larger exposures can produce permanent A-mode signal changes. We are optimizing this technique to enable it to validate therapy beam placement, to evaluate induced compression of vessels, and to monitor tissue rigidity changes that accompany thermal-lesion formation.

\section{$10: 55$}

2aBB9. SonablateTM 500: A novel ultrasound platform for transrectal image-guided treatment of prostate diseases. Narendra T. Sanghvi, Roy F. Calson, Ralf Seip, Jahangir Tavakkoli (1 Focus Surgery, Inc., Indianapolis, IN 46226), Kris A. Dines (XDATA Corp., Indianapolis, IN 46220), Thomas A. Gardner (Indiana Univ. Med. School, Indianapolis, IN 46202), and Toyoaki Uchida (Kitasato Univ. Med. School, Sagamihara, Japan)

The SonablateTM system for transrectal image-guided high intensity focused ultrasound (TR-HIFU) treatment of prostate diseases has been in clinical use since 1992. Recently, we developed the third generation of the system, call SonablateTM 500, with major improvements aimed specifically toward localized prostate cancer treatment. Salient features of the SonablateTM 500 include: (1) PC-based digital technology, (2) multifocal lengths probe with split beam transducer to cover a wide range of tissue depths (2.5-4.5 $\mathrm{cm}$ ), (3) an upgraded treatment planning and control software with safety features such as reflectivity index measurement (RIMTM) and transducer tissue measurement (TTMTM); and (4) 3-D volume image rendering of the prostate. The SonablateTM 500 platform was tested in an in vivo canine experimental study in which the entire prostate was ablated. The prostate ultrasound images before and post HIFU treatment show very good correlation to the planned lesion. Histology delineated that the entire prostate was contiguously ablated without affecting surrounding tissues. Long-term canine survival studies as well as human prostate cancer studies under the FDA's IDE (Investigational Device Exemption) approval are in progress. [We would like to thank the engineering and clinical staff of Focus Surgery, Inc. who made this project possible.]

\section{1:15}

2aBB10. HIFU for tumor therapy in China: A report on its application to humans for a variety of diseases. Feng Wu and Wang Zhibiao (Box 153, Inst. of Ultrasound Eng. in Medicine, Chongqing Medical Univ., 1 Medical College Rd., Chongqing 400016, PROC)

Starting in 1997, our center began to apply HIFU in clinical treatment to patients with malignant solid tumor, such as breast cancer, hepatic carcinoma, and bone sarcoma, etc. During three years of study, we have observed satisfactory results from more than 400 cases, including some patients with bone sarcomas. A brief description of our treatment methodology will be reviewed as well as a few individual cases.

11:35-12:30

Panel Discussion 


\title{
Session 2aEA
}

\section{Engineering Acoustics: Small Scale Acoustics and Acoustic Devices}

\author{
Daniel M. Warren, Chair \\ Knowles Electronics, 1151 Maplewood Drive, Itasca, Illinois 60143
}

Chair's Introduction-8:15

Invited Papers

8:20

2aEA1. A silicon nitride microphone diaphragm inspired by the ears of the parasitoid fly Ormia ochracea. Ronald N. Miles, Colum Gibbons, Jia Gao (Dept. of Mech. Eng., State Univ. of New York, Binghamton, NY 13902-6000, miles@binghamton.edu), Kyutae Yoo, Quang Su (Cornell Univ., Ithaca, NY 14853), and Weili Cui (State Univ. of New York, Binghamton, NY 13902-6000)

A differential microphone is described that has been designed to employ similar operating principles to that of the ears of the parasitoid fly, Ormia ochracea. The ears of this fly have been shown to be highly directional even though they are only about $1 \mathrm{~mm}$ across [R. N. Miles, D. Robert, and R. R. Hoy, J. Acoust. Soc. Am. 98, 3059-3070 (1995)]. Analyses of the mechanics of this biological system suggest novel approaches to the design of miniature directional microphones. Finite element analysis results for the acoustic resonse of a $1 \mathrm{~mm}$ by $2 \mathrm{~mm}$ silicon nitride microphone diaphragm are presented. The diaphragm responds to pressure gradients in a manner that is inspired by Ormia's ears. Predicted results for the natural frequencies, mode shapes, frequency response and directivity of our design are shown to compare closely with measured data obtained for a prototype silicon nitride diaphragm. [Work supported by NIH and DARPA.]

\section{8:40}

2aEA2. Wireless telephone design considerations. Robert A. Zurek (Personal Communs. Sector Res. and Adv. Technol. Labs, Motorola, 2001 N. Division St., Harvard, IL 60033,w15754@email.mot.com)

As wireless communication devices have shifted from novelty devices to everyday use, the demands on the performance and quality of the device's audio and acoustic systems has increased. The acoustic portion of wireless phone design can no longer be taken for granted as the size of the devices decrease and the expectations increase. While wireless telephones share many of the design challenges of wired landline systems, they also present additional challenges not normally found in landline systems. The shape and size of today's phones make past assumptions used in the design of landline systems invalid, such as the assumption that the acoustic load on the receiver is constant. The delay inherent in today's digital systems coupled with the small size of handsets has led to an increased awareness of acoustic feedback from the receiver to the microphone, which results in an undesirable system echo. In addition to the receiver design and echo concerns is the necessity of designing in an alert or ringer that must produce adequate SPL levels to inform the user of a call, while fitting in a very small space. The aforementioned issues will be discussed along with current design techniques utilized to overcome these challenges.

9:00

2aEA3. Scaling issues in a submillimeter MEMS microphone. Peter V. Loeppert (Emkay Innovative Products, 1151 Maplewood Dr., Itasca, IL 60143)

A silicon based microphone with a $0.5 \mathrm{~mm}$ diam diaphragm has been developed using a combination of surface and bulk micromachining or MicroElectro Mechanical Systems (MEMS) techniques. This device is intended for high volume general purpose markets such as telephony, where cost is a key factor. In batch fabricated MEMS devices, cost is critically determined by the device size. Yet the performance standards for this microphone are set by relatively large electret condenser microphones (ECM) built in competing technologies. This paper will discuss the scaling issues involved in meeting high performance goals in a very small MEMS device. In particular, tradeoffs made to achieve high sensitivity, low noise, and unconditional stability will be discussed.

9:20

2aEA4. Turbulence induced distortion in hearing aid receivers. Daniel M. Warren (Knowles Electronics, LLC, 1151 Maplewood Dr., Itasca, IL 60143, daniel.warren@knowles.com)

The quality of resonances in hearing aid receivers can be reduced by adding damping in various places in the electroacoustic system. Acoustic damping may be implemented by means of tiny $(\approx 0.001$ in. diam $)$ holes in the diaphragm separating the front and rear cavities of the receiver. The holes are very small, $\approx 1 / 10$ of the boundary layer thickness. As noted previously [P. M. Morse and K. U. Ingard, Theoretical Acoustics (Princeton University Press, Princeton, 1968)], "fortunately, such a minute hole is of no practical 
interest." However, in hearing aid receivers such holes are of practical interest, and are identified as causing high distortion at frequencies below $500 \mathrm{~Hz}$. The experiment described demonstrates that the distortion is caused by turbulence in air flow through holes in the diaphragm. The experiment also shows that distributing the air flow among multiple smaller holes raises the threshold for turbulent flow, alleviating distortion at the nominal drive level. [Work supported by Knowles Electronics, LLC.]

\section{Contributed Papers}

\section{9:40}

2aEA5. Challenges for the commercialization of acoustic sensors using microfabrication technologies. Michael Pedersen (CNRI, 1895 Preston White Dr., Ste. 100, Reston, VA 20191, pedersen@mems-exchange.org)

In this paper a review is given of the methods used in the design and realization of acoustic sensors based on microfabrication technologies. It is shown that the most important issues relate to material properties, which must be tightly controlled to achieve acceptable consistency and stability of the devices. In addition, the requirements for successful commercialization of such devices are discussed and a number of critical parameters are identified which include environmental performance, reliability and cost. It is contended that microphones made with this new technology will not provide advances over existing technology in terms of sensitivity and noise performance, and hence the motivation for the use of the technology must be found in the added value of smaller size, increased bandwidth, and better flexibility in terms of device assembly and ruggedness. Based on these considerations a new capacitive microphone design is proposed, which simplifies the fabrication of the so-called free-plate or zero-stress microphone, referring to the lack of tension in the microphone diaphragm. It is further shown that the introduction of flip-chip assembly methods will enhance the repeatability and ruggedness of the microphone package, which so far has received little attention in the development micromachined acoustic sensors.

\section{9:55-10:10 Break}

\section{0:10}

2aEA6. FEM based simulation of nonlinear effects in armature alerts. Robert A. Zurek and Simone Koo (Personal Communs. Sector Res. and Adv. Technol. Labs, Motorola, 2001 N. Division St., Harvard, IL 60033, w15754@email.mot.com)

Although alerts or ringers have been used in wireless phones and pagers since their inception, design of these systems in the past has been limited to estimation of the Helmholtz resonance of the acoustic system followed by trial and error modification until the desired alert level is achieved. With the advent of reasonably fast Vibro-Acoustic Boundary Element and Finite Element solvers, a much more accurate and inclusive method of alert system design is possible. BEM and FEM alert models can take into account the baffling effects of the phone or pager case, losses due to surface absorption and transmission loss, geometrical characteristics of the system's ports and cavities, and the frequency dependent vibration characteristics of the transducer. These simulation capabilities, combined with Doppler laser vibrometer based alert characterization, provide for an improved alert design cycle. This paper will discuss some of the new alert characterization and simulation techniques used in the Motorola PCS Research Advanced Products Lab. The results of this technique will be compared to measured data and earlier simulation attempts, and future enhancements to the current technique will also be discussed.

\section{0:25}

2aEA7. New materials for thermal microphone sensors. Jerry Helffrich and Heather Hanson (Southwest Res. Inst., 6220 Culebra Rd., Bldg. 178, San Antonio, TX 78238-5166, jhelffrich@swri.org)

Existing thermal microphone sensors use MEMS technology to fabricate two beams. These initially heated beams cool with the movement of air in a sound wave. The temperature difference between the two beams due to convective heat transfer can be converted to an air velocity measurement. These microphones have the advantage of an extremely small size and dipole sensing, but suffer from a low signal-to-noise ratio, lowfrequency response, and high-power dissipation. We are investigating the use of new, high-temperature coefficient of resistivity (TCR) materials for thermal microphones. This second generation of devices should deliver an improved response than those that are commercially available. Measurements of the response of thermal microphones using new resistive materials will be presented.

\section{0:40}

2aEA8. Thermoelastically actuated MEMS ultrasonic transducer. Venkataraman Chandrasekaran (Dept. of Aerosp. Eng., Mech., and Eng. Sci., Univ. of Florida, Box 116250, Gainesville, FL 32611), Xi Li, Jian Li, Toshikazu Nishida (Dept. of Elec. and Computer Eng., Univ. of Florida, Box 116130, Gainesville, FL 32611), Louis N. CattafestaIII, and Mark Sheplak (Dept. of Aerosp. Eng., Mech., and Eng. Sci., Univ. of Florida, Box 116250, Gainesville, FL 32611)

Ultrasound based techniques provide a method for noncontact distance measurement utilizing a thin vibrating diaphragm as the acoustic source. Devices based on different operating principles have been reported including electrostatic and piezoelectric. The conventional pulse-echo technique is limited by a finite pulse decay time that restricts the measurement range to greater than a few centimeters. Proximity sensing on the order of millimeters can be achieved using continuous wave form techniques such as the acoustic impedance method or simultaneous excitation at multiple frequencies. Using silicon microfabrication technology, a MEMS ultrasonic transducer has been fabricated for millimeter range proximity sensing. The salient features of the sensor/actuator include a $0.01 \mathrm{~mm}$ thick, $1 \mathrm{~mm}$ diameter composite diaphragm that is thermoelastically driven at ultrasonic frequencies using a circular heater at its center. Dynamic Joulean heating of the diffused surface heater induces bending moments in the diaphragm due to the temperature gradient across the membrane cross section. Electromechanical transduction of the membrane vibration is achieved via four diffused silicon piezoresistive strain gauges located at the edge of the diaphragm. The design, fabrication and preliminary characterization of this sensor/actuator are presented.

\section{0:55}

2aEA9. Measurement of particulate densities in air. Ravinder Beniwal and Sean Wu (Dept. of Mech. Eng., Wayne State Univ., 5050 Anthony Wayne Dr., Detroit, MI 48202)

Clean air is one of the most important issues that govern the health of all live forms. However, presently there are not many quick and simple methods for measuring impurities like particulates in air. These impurities have an enormous diversity in their physical and chemical structure. They may be unburned carbon particles from a diesel engine exhaust and chimney, pollen grains in the spring air, or asbestos in a factory. This paper shows that changes in the composition of the air cause a change in the speed of sound. Therefore by measuring the change in the speed of sound, it is possible to monitor the density of particulates in the air. Preliminary tests are conducted on various smoke-air mixtures. The results demonstrate that this methodology is very sensitive to any changes in the composition of the air. Its implementation is very simple and efficient, and costs much less than the conventional method currently used in the auto industry. This technique will be used to calculate the mass density of the particulates resulting from a diesel engine, and results thus obtained will be compared with those calculated using other methods. 
2aEA10. Capacitive micromachined interdigital transducers. Goksen G. Yaralioglu, Mohammed H. Badi, and Butrus T. Khuri-Yakub (Ginzton Lab., Stanford Univ., Stanford, CA 94305)

Capacitive Micromachined Ultrasonic Transducers (CMUTs) have become very popular for medical imaging and sonar applications during the last decade. They can compete with piezoelectric transducers in terms of efficiency and bandwidth. Recently, it has been noticed that these devices couple energy into silicon wafer in addition to the immersion medium. By placing the CMUTs on a substrate in an interdigitated configuration, Lamb wave or Rayleigh wave modes can be excited with very high efficiency without a need for any piezoelectric material. In this study, the acoustic power coupled into the silicon substrate as well as the acoustic impedance of the CMUT membrane are calculated by using finite element analysis. Normal mode theory is used to find the distribution of the acoustic power among different Lamb wave modes. For low frequency $(1 \mathrm{MHz})$ devices, the lowest order antisymmetric $\left(A_{0}\right)$ mode Lamb wave is the dominant mode in the substrate. For high frequency devices $(100 \mathrm{MHz})$, interdigital CMUTs excite Rayleigh wave with efficiencies comparable to piezoelectric surface acoustic wave (SAW) devices. Experimental results for Lamb wave devices in the vicinity of $1 \mathrm{MHz}$ will also be presented. [Work supported by ONR.]
2aEA11. Difference method for measuring the vibration sensitivity of microphones. Anthony J. Brammer (Inst. for Microstructural Sci., Natl. Res. Council, Montreal Rd., Ottawa, ON K1A 0R6, Canada)

In some applications, microphones are subjected to sufficient vibration that their vibration response is of interest (e.g., feedback from earphone to microphone in wireless telephones, and hearing aids). A procedure for measuring this microphone property should take into account the airborne sound produced by the apparatus. In the present method, two microphones of the same type are mounted facing each other with diaphragms parallel, and form the plane, circular ends of a cylindrical enclosure. The dimensions of the air-filled volume between the two microphones are chosen to ensure that both microphones experience the same sound pressure. When the enclosure is vibrated in a direction normal to the plane of the microphone diaphragms (i.e., along the long axis of the cylinder), the vibration responses of the two microphones are essentially out of phase. The difference signal between the two microphone outputs will then provide a measure of the mean vibration response of the microphones from which the common airborne response of the microphones has been subtracted. The extent to which the response to sound within the apparatus interferes with the measurement of the vibration response of individual microphones may then be estimated. Results will be presented for a miniature electrical microphone.

\title{
Session 2aED
}

\section{Education in Acoustics: Hands-on Demonstrations for High School Students}

\author{
Uwe J. Hansen, Chair \\ Department of Physics, Indiana State University, Terre Haute, Indiana 47809
}

Chair's Introduction-10:00

This session is intended to communicate the excitement of "doing" acoustics to high school students in the South Florida area. Individual demonstrations with associated instrumentation will be presented in a brief introduction. In the subsequent hands-on session, presenters will guide individual students to discover the fun of acoustics by letting them "twiddle the knobs." Topics available for student participation will range in sophistication from simple wave observation to normal mode mapping on musical instruments. While attendance as well as participation by conference attenders is welcome, hands-on participation is primarily designed for the students attending this session by special arrangement. 


\title{
Session 2aMU
}

\section{Musical Acoustics: Musical Instrument Acoustics}

\author{
James P. Cottingham, Chair \\ Physics Department, Coe College, Cedar Rapids, Iowa 52402
}

\section{Contributed Papers}

\section{8:00}

2aMU1. Shape optimization techniques for musical instrument design. Luis Henrique (Instituto Politécnico do Porto, Escola Superior de Música e das Artes do Espectáculo, R. da Alegria, 503, 4000-046 Porto, Portugal), José Antunes (Instituto Tecnológico e Nuclear, Appl. Dynam. Lab., 2686 Sacavém Codex, Portugal), and João Soeiro de Carvalho (Universidade Nova de Lisboa, Faculdade de Ciências Sociais e Humanas, Av. de Berna 26-C, 1050-069 Lisboa, Portugal)

The design of musical instruments is still mostly based on empirical knowledge and costly experimentation. One interesting improvement is the shape optimization of resonating components, given a number of constraints (allowed parameter ranges, shape smoothness, etc.), so that vibrations occur at specified modal frequencies. Each admissible geometrical configuration generates an error between computed eigenfrequencies and the target set. Typically, error surfaces present many local minima, corresponding to suboptimal designs. This difficulty can be overcome using global optimization techniques, such as simulated annealing. However these methods are greedy, concerning the number of function evaluations required. Thus, computational times can be unacceptable if complex problems, such as bell optimization, are tackled. Those issues are addressed in this paper, and a method for improving optimization procedures is proposed. Instead of using the (local) parameters $P_{f}(\mathbf{r})$ as searched variables, the system geometry is modeled in terms of truncated series of orthogonal space-functions $P_{j}(\mathbf{r})=\Sigma_{n} a_{j n} \psi_{j n}(\mathbf{r})$, and optimization is performed on the (global) coefficients $a_{j n}$. Fourier series and orthogonal polynomials are typical functions $\psi_{j n}(\mathbf{r})$. This technique reduces considerably the number of search variables, and has a potential for significant computational savings in complex problems. It is illustrated by optimizing the shapes of both current and uncommon marimba bars.

\section{$8: 15$}

2aMU2. Violin normal mode radiative properties. George Bissinger and John Keiffer (Phys. Dept., East Carolina Univ., Greenville, NC 27858)

Calibrated far-field pressure measurements of violin radiation excited by force hammer impact at the G-string corner of the bridge were performed at 266 microphone positions over an $r=1.2 \mathrm{~m}$ spherical surface inside an anechoic chamber. These were combined with simultaneous mobility measurements over the violin surface using a scanning laser to extract radiation efficiencies for violin normal modes below $4000 \mathrm{~Hz}$. For favorable cases it was possible to compute experimental values for radiation damping, and the companion fraction of vibrational energy lost to acoustic radiation. As an example, preliminary results for the lower of the first corpus bending modes of a violin by A. Schroetter were the following: radiation efficiency of $\sim 0.12$, total damping of $\sim 1.15$ (percent critical) for a $\mathrm{Q}$ of $\sim 43$, radiation damping of $\sim 0.41$ (percent critical). For this mode $\sim 35 \%$ of the vibrational energy was radiated as sound. Radiation patterns measured for each mode were corrected for anechoic chamber reflection/interference effects. [Research supported by the NSF DMR9802656.]
$8: 30$

2aMU3. How important are torsion modes in bowed-string dynamics? Octávio Inácio (Instituto Politécnico do Porto, Escola Superior de Música e das Artes do Espectáculo, R. da Alegria, 4000-046 Porto, Portugal), José Antunes (Instituto Tecnológico e Nuclear, Appl. Dynam. Lab., 2686 Sacavém Codex, Portugal), and Luis Henrique (Instituto Politécnico do Porto, Escola Superior de Música e das Artes do Espectáculo, R. da Alegria, 503, 4000-046 Porto, Portugal)

In spite of the venerable history of bowed-string research, several aspects are still inadequately clarified. The importance of torsion modes on the motion regimes of bowed strings is one such issue. Experiments involving torsion are difficult to perform and most of the results available pertain to numerical simulations. The authors' approach differs from previous efforts in two main aspects: (1) the development of a computational method quite distinct from the classic wave-propagation approach pioneered by McIntyre, Schumacher, and Woodhouse and (2) an extensive and systematic analysis of the coupling between torsion and transverse motions. The numerical simulations are based on a modal representation and a friction model that enable accurate representations of the stick-slip friction forces and of the string dynamics, in both time and space. Many relevant aspects of the bowed-string can be readily implemented, including string inharmonic behavior, finite bow-width and torsion effects. Concerning the later aspect, a realistic range of the wave-speed ratio $\alpha$ $\equiv C_{\text {Tors }} / C_{\text {Tran }}=2 \sim 7$ is investigated, for several values of the bow velocity and normal force. The results suggest that torsion modes can affect both transient durations and steady state regimes, in particular when $\alpha$ $<4$. Gut strings should then be particularly prone to torsion effects.

\section{$8: 45$}

2aMU4. Comparisons of theoretical and experimental transverse motion for a plucked, stiff piano string. José Sánchez and James Irwin, Jr. (Dept. of Elec. and Computer Eng., Bradley Univ., 1501 W. Bradley Ave., Peoria, IL 61625)

This work is meant to determine whether central or backward difference approximations in computer simulations more closely approximate reality. The stiff piano string motion is experimentally measured by a real-time analog optical technique that was developed to measure the onedimensional transverse displacement of a plucked string. The optical technique uses a detector that collects laser light not obstructed by the string. The received light is converted to a voltage, which is related to the string displacement perpendicular to the laser beam. The signal is then filtered and conditioned by external circuitry, digitized by a Mona data acquisition system, and are displayed using Cool Edit Pro. These measurements are then compared to two different finite difference computer simulations. One of the computer simulations uses central differences and the other uses backward differences, both with the appropriate boundary conditions and parameters. The comparison of the experimental data and the computer simulations is comprised of informal listening tests, spectral, and time domain comparisons. 
2aMU5. The HANG: A hand played steel drum. Thomas D. Rossing (Phys. Dept., Northern Illinois Univ., DeKalb, IL 60115), Uwe J. Hansen (Indiana State Univ., IN), Felix Rohner, and Sabina Schärer (PanArt, Bern, Switzerland)

The HANG is a new steel instrument consisting of two spherical shells of steel, suitable for playing with the hands. Seven to nine notes are harmonically tuned around a central deep note which is formed by the Helmholtz resonance. Elliptical note areas are formed on the upper shell and tuned so that the $(1,1)_{\mathrm{a}}$ and $(1,1)_{\mathrm{b}}$ modes of each note area have frequencies that are in the ratios of $3: 2: 1$ with respect to the fundamental, except in the highest notes where they are in the ratio of $2.5: 2: 1$ or $2.4: 2: 1$. Holographic studies show the modal shapes and also the extent of coupling between note areas.

\section{9:15}

2aMU6. Acoustic reflectometry in the manufacture of reproductions of historical wind instruments. D. Murray Campbell, James M. Buick, Jonathan A. Kemp, and Maarten O. van Walstijn (Dept. of Phys. and Astron., Univ. of Edinburgh, James Clerk Maxwell Bldg., Edinburgh EH9 3JZ, U.K., D.M.Campbell@ed.ac.uk)

Acoustic reflectometry has become a well-established technique for investigating the internal bore profile of cylindrically symmetrical ducts. Although the method has previously been applied with some success to the study of musical wind instrument bores, there are practical problems which limit the accuracy of bore reconstruction of instruments which are long or widely flaring, or which have side finger holes. This paper reports on a collaboration with Christopher Monk Instruments Ltd., a Londonbased firm specializing in the reproduction of cornetts and serpents. These are lip-reed instruments with side finger holes, and a curved bore center line which inhibits the use of conventional bore measuring techniques. It is demonstrated that acoustic reflectometry can provide bore profiles of these instruments with sufficient precision to be a valuable guide in their construction or repair. The precautions necessary to achieve this level of accuracy are described, and prospects for further refinements are discussed. [The collaboration of Keith Rogers and Jeremy West of CMI Ltd. is gratefully acknowledged. Work supported by the Engineering and Physical Sciences Research Council, U.K.]
2aMU7. Acoustical analysis of an ancient Mexican flute. Rolando Menchaca (CIC, IPN, Mexico), Sergio Beristáin (Lab. Acustica, ESIME, IPN, Mexico and and P.O. Box 75805, 07300, Mexico City, Mexico), and Roberto Velazquez (CIC, IPN, Mexico)

The acoustical anslysis made on one flute taken from a given class among very old Mexican flutes is discussed. First, some acoustical characteristics of the sounds produced from this musical instrument are presented, such as the acoustic power and the energy spectrum of some of the different sounds generated by the flute. Then an analysis is made on the acoustical details of the construction technology used to build this kind of instrument, like acoustical mass and compliance, turbulence generation, radiation from the tuning holes, and so on. And some discussion on the probable design objectives on the mind of the ancient Mexicans when they developed and built this sound instrumentation, as the possibility that natural sounds were taken as a reference for their art evolution.

\section{$9: 45$}

2aMU8. On the acoustics of organ reed pipes. Eric Cox and Thomas D. Rossing (Dept. of Phys., Northern Illinois Univ., DeKalb, IL 60115)

In most lingual organ pipes, the reed vibrates against a fixed shallot and modulates the flow of air passing through the shallot into the resonator. In an earlier paper [T. D. Rossing, J. Angster, and A. Miklos, paper 2pMU6, 136th meeting, Acoustical Society, 1998] we described distinct regimes of oscillation observed in a Trompette pipe. Using a laser vibrometer, we have now observed similar phenomena in two pipes of quite different design: a Rankett and a Krummhorn. Without the resonator the reed frequency in each pipe varies smoothly with reed length, but the resonator lowers the frequency of the reed and also produces distinct regimes of sound, separated by discontinuities, as the system jumps from one regime to the next one. Changing the wind pressure has a modest effect on sound output but little effect on sounding frequency, within limits.

\title{
Session 2aNS
}

\section{Noise, Architectural Acoustics and Signal Processing in Acoustics: Implementing the Classroom Acoustics Standard}

\author{
John Erdreich, Chair \\ Ostergaard Acoustical Associates, 200 Executive Drive, West Orange, New Jersey 07052
}

Chair's Introduction-8:30

Invited Papers

8:35

2aNS1. Acoustical environments measured in urban and suburban schools. John Erdreich (Ostergaard Acoust. Assoc., 200 Executive Dr., West Orange, NJ 07052)

Measurements of sound levels in numerous classrooms and other instructional spaces in primary educational facilities over the past several years have revealed a lack of acoustical control within public schools. We have found environments in excess of NC-60 more often than we have found NC-30 rooms in unoccupied spaces. Occupied sound levels in instructional spaces may exceed 90 
$\mathrm{dB}(\mathrm{A})$. Because of poor acoustical design, we have found classrooms on the same floor with background sound levels that vary from NC-60 to NC-25, depending on the distance from the HVAC mechanical room. This paper will present some case studies that demonstrate the need for an acoustical standard for the classroom.

9:05

2aNS2. Development of an American National Standard for classroom acoustics. David Lubman $\quad$ (D. Lubman \& Assoc., 14301 Middletown Ln., Westminster, CA 92683-4514) and Louis C. Sutherland (Acoust. Consultant, 27803 Longhill Dr., Rancho Palos Verdes, CA 90725-3908)

A draft ANSI (American National Standards Institute) Standard for classroom acoustics will be submitted for approval this year. It is believed that adopting this Standard will result in a more inclusive and effective education system. Although eliminating acoustical barriers benefits everyone, young children and persons with hearing, language, speech, and learning disabilities are special beneficiaries. Titled "Acoustical performance criteria, design requirements, and guidelines for schools," the draft, designated ANSI S12.60-200X, limits noise and reverberation in classrooms and imposes minimum values of sound isolation between enclosed classrooms and adjacent spaces. The effort began when many members of the Acoustical Society of America (ASA) became convinced that noise and reverberation in classrooms were serious barriers to learning. The ASA organized a Task Force on classroom acoustics, assembling volunteer scientists and engineers from architectural acoustics, noise, speech, and psychological acoustics. Task Force outreach seminars led to a broad coalition of individuals, and public and private organizations. They determined that a national Standard for classroom acoustics was needed. To develop that Standard, ANSI Working Group 42 on classroom acoustics was established and scoped. Draft 10 was successfully balloted in the S12 Committee in January 2001. In this paper we discuss the development of the Standard.

\section{9:35}

2aNS3. Experiences with acoustical renovation of classrooms in a public school. Robert Wallace (The Harbor Science and Arts Charter School, New York, NY 10029; robertw104@aol.com)

The Harbor Science and Arts Charter School is a small, public elementary school with children in grades 1 through 7 located in the East Harlem section of New York City. A hallmark of our curriculum is that we encourage students to work on group projects and to make many oral presentations of the results of their work. We noticed that when children were engaged in group work and oral presentations, it was often difficult to understand everything that was said. One of our fourth grade teachers had a friend who was an acoustical designer who arranged a sound analysis of the teacher's room that confirmed that our high ceilings, concrete block walls, and window air conditioners were creating some of the problems we were experiencing. Using the room as a test case, Armstrong Industries donated materials for modifications to the room. They subsidized lowering the ceiling with acoustical tiles. We installed new bulletin boards and purchased new air conditioners. The results were extraordinary. Students and teachers who used the classroom were amazed at the improvements. We have used what we have learned to modify all of the classrooms as well as the cafeteria. These relatively simple modifications have made a significant improvement in the quality of life in our school.

10:05-10:20 Break

10:20

2aNS4. Implementation of ANSI S12.60 acoustical performance criteria, design requirements and guidelines for schools. Lois E. L. Thibault (Coordinator of Res., U.S. Access Board, 1331 F St. NW, \#1000, Washington, DC 20004-1111, thibault@access-board.gov)

In 1997, the parent of a child with a hearing loss petitioned the U.S. Access Board to include guidelines for classroom acoustics in the accessibility guidelines the Board maintains under the ADA. The Board agreed that poor listening conditions in schools could be a barrier to the education of children with hearing impairments and other disabilities and arranged to collaborate with ASA and other stakeholders on an acoustical standard for classrooms. The Board will submit the completed standard to the International Codes Council for reference in the International Building Code adopted by many states and local jurisdictions, making it enforceable through the local permitting and inspections process. Reference in the ADA Accessibility Guidelines may follow in its next review cycle in 2005. Individualized Education Plans (IEPs) required under IDEA (Individuals with Disabilities Education Act) may also reference the new standard.

\section{0:50}

2aNS5. The new standard's HVAC background noise requirements and their implementation in building design. Mark E. Schaffer (Schaffer Acoust., Inc., 869 Via de La Paz, Ste. A, Pacific Palisades, CA 90272) and Jerry G. Lilly (JGL Acoust., Inc.)

The new ANSI classroom standard recommends long-term, steady-state background sound level limits of $35 \mathrm{dBA}$ and $55 \mathrm{dBC}$. Since the HVAC system is typically a room's dominant steady-state noise source, it is usually reasonable to apply these limits to the design of the system, assuming that other noise sources produce insignificant additions to the room's long-term background noise environment. The recommended sound level limits are significantly lower than current industry standards, and complying with them will increase system costs. School administrators, architects, and HVAC system designers should, therefore, be prepared for the changes. This paper summarizes equipment selection and system design guidelines that have been found to be necessary for ensuring compliance with the sound level limits. 
2aNS6. The Future of Schools, Inside and Out: TV documentary. Debra Greene and Crystal Edgington (Information Television Network, 621 NW 53rd St., Boca Raton, FL 33487)

"The Future of Schools, Inside and Out" is an information-rich and colorful 1 hour documentary designed to entertain and inform viewers about America's schools. Areas of interest include acoustics, building safety, energy efficiency, day lighting, and more. The episode explores the connection between our nation's school facilities and the quality of a child's education. This program travels out in the field to school locations from rural areas to the inner city to the suburbs to help tell the story. Several top experts not only point out the problems and challenges but also offer solutions to help give the children of America every possible advantage in their learning environment.

\title{
Session 2aPA
}

\author{
Physical Acoustics: High Amplitude Effects in Resonators I \\ Anthony A. Atchley, Cochair \\ Graduate Program in Acoustics, Pennsylvania State University, P.O. Box 30, Applied Science Building, \\ State College, Pennsylvania 16804 \\ Victor W. Sparrow, Cochair \\ Graduate Program in Acoustics, Pennsylvania State University, P.O. Box 30, Applied Science Building, \\ State College, Pennyslvania 16804
}

Invited Papers

8:00

2aPA1. Flow and temperature fields in thermoacoustic stacks. Ph. Blanc-Benon and S. Duffourd (Ecole Centrale de Lyon, Ctr. Acoustique, LMFA UMR CNRS 5509, BP 16369131 Ecully Cedex, France, Philippe.Blanc-Benon@ec-lyon.fr)

The paper describes the flow patterns and the temperature distribution measured in a thermoacoustic engine. Experiments have been performed in a resonant standing wave thermoacoustic refrigerator model. The knowledge of temperature and flow fields in the microchannels and at the edges of the stack plates becomes an increasingly important issue in the design of heat exchangers for thermoacoustic engines. Different geometries of the stack plates have been investigated as well as at different pressure levels. Heat exchangers have also been placed on both sides of the stack, with adjustable gaps. 2-D particle image velocimetry was used to investigate the flow with enlarged observation fields near the ends of the stack plates. We present velocity profiles across the microchannels, 2-D velocity fields. Vorticity maps were deduced using a criterion based on a normalized angular momentum. Vortex motion close to the stack ends has been clearly observed, however, with a strong influence of the plate thickness. An array of miniaturized thermocouples permits us to follow in real time the building-up of the temperature gradient along the stack for different oscillating flow conditions and different gaps between the stack and the heat exchangers. Finally, comparisons are made with theoretical models and numerical simulations recently published in the literature. [Work supported by DGA.]

2aPA2. Numerical simulation of viscous losses in resonators. Philip J. Morris and Said Boluriaan (Dept. of Aerosp. Eng., Pennsylvania State Univ., 233 Hammond Bldg., University Park, PA 16802)

Thermoacoustic devices consist of resonators that contain high amplitude standing or traveling waves. They depend on the interaction between the high amplitude acoustic waves with surfaces in the resonator, such as a stack, to either transport heat or extract power. However, this same interaction results in losses. These losses may be in the form of viscous dissipation or from the conversion of acoustic energy into vorticity. The latter occurs at sudden changes in the cross sectional area of the resonator where the unsteady flow produces a jet- and sinklike behavior in one period of the oscillation. Numerical simulations are performed on a parallel cluster of PC's for the unsteady flow generated at such a sudden change of area. Results are presented for the instantaneous and averaged flow field and the thermodynamic properties. The pressure drop across the sudden change in area is calculated and the physical processes that result in this pressure difference are examined. Estimates are given of the total losses in the resonator and the relative contributions of viscous dissipation in the wall boundary layers and the conversion of acoustic energy into vorticity are given. [Research supported by ONR.] 
2aPA3. Minor losses in oscillatory flow. Barton L. Smith and Gregory W. Swift (Condensed Matter and Thermal Phys. Group, Los Alamos Natl. Labs., Los Alamos, NM 87545)

Energy dissipation that is manifested by gradients in pressure in a flow system is often expressed in terms of a pressure loss factor of the form $K=\Delta 2 P / \rho v^{2}$. While there is a wealth of data on loss factors for a myriad of geometries in steady flow, little such data exists for pulsatile or oscillatory flow. Recent advances in thermoacoustics, which involves heat engines and refrigerators with no moving parts, motivate work in this area. An oscillatory flow system has been constructed to investigate loss factors in 2D geometries. The current results provide loss factors of entrances/sudden expansions. It is found that the losses are primarily a function of the displacement amplitude. The results also provide information on the variation of the "equivalent length" with the same parameter, and on turbulent dissipation in the channel. Oscillating flow in diffusers is also investigated, with emphasis on loss factors as a function of diffuser angle, and unsteady separation effects. [Work supported by the Los Alamos National Laboratory LDRD funds.]

\section{9:30-9:45 Break}

\section{Contributed Papers}

9:45

2aPA4. Measurements of acoustic Einstein-Hopf drag on objects undergoing volume oscillations. Edward Tucholski and Andres Larraza (Naval Postgrad. School, Phys. Dept., Monterey, CA 93943, larraza@physics.nps.mil)

It has been shown theoretically that the drag on an object undergoing volume oscillations can be modified by the presence of isotropic, homogeneous, broadband acoustic noise, when the band overlaps the objects resonance width [Larraza and Tucholski, Phys. Rev. Lett. 84, 2378-2380 (2000)]. These results constitute an acoustic analog to the Einstein-Hopf drag on an oscillating dipole in the presence of electromagnetic fluctuations. However, an important difference is that band-limited acoustic noise can reduce the drag when the lower frequency of the spectrum coincides with the resonant frequency of the object. Experimental results of the drag measured on bubbles and aerogel spheres moving in an band-limited noise field are reported.

10:00

2aPA5. Liquid droplet generation utilizing high intensity acoustic standing waves within shaped resonators. Josh Roby, Tim Cameron, and Bart Lipkens (Dept. of Mech. Eng., Virginia Commonwealth Univ., 601 W. Main St., Richmond, VA 23284-3015 and Dept. of Psych., Boston Univ., Boston, MA 02115)

Generating liquid droplets from a bulk liquid underlies a vast number of process related technologies ranging from fuel combustion to respiratory drug delivery. Resonant Macrosonic Synthesis (RMS) is a technology that makes possible the generation of very high-amplitude shock-free acoustic standing waves within shaped resonators. Sound pressure levels in excess of $190 \mathrm{~dB}$ have been measured within RMS resonators. Bulk liquids subject to these high intensity oscillating acoustic waves are drawn into the acoustic field and atomized. Under continuous processing conditions water droplets on the order of $30 \mu \mathrm{m}$ Sauter Mean Diameter have been generated with RMS. Droplet size measurements were obtained using a laser diffraction particle-sizing instrument. Data indicate that under all but the lowest liquid flow rates, liquid accumulates within the acoustic resonator, altering the processing characteristics. As liquid accumulation increases under fixed drive amplitude the power delivery, acoustic pressure, and acoustic resonance frequency drop, which coincides with increased mean particle diameters. Therefore the mean droplet diameters increase with an increasing liquid flow rate. Experiments have shown, however, that these characteristics are highly dependent on the process entry and removal points on the resonator.
10:15

2aPA6. Simulation of streaming in a tapered resonator. Brian C. Tuttle and Victor W. Sparrow (Grad. Prog. in Acoust., Penn State, 217 Appl. Sci. Bldg., University Park, PA 16802)

In 1997, Olson and Swift [Cryogenics 37, 769-776 (1997)] investigated acoustic streaming in conical tubes of varying taper angle of a pulse tube refrigerator to increase the efficiency. They found that an optimal taper angle existed that would minimize Rayleigh streaming in the tube. The present research is aimed at numerically simulating this taper effect on the Rayleigh streaming. The two-dimensional (2-D) nonlinear acoustic numerical approach of Sparrow and Raspet [J. Acoust. Soc. Am. 90, 2683-2691 (1991)] is modified for tapered geometries matching those of Olson and Swift. The method for grid generation and stretching will be discussed. Results of this numerical simulation will be compared to the published experimental results and will aid in a deeper understanding of acoustic streaming. [Work supported by ONR.]

10:30

2aPA7. Application of a stream-function numerical approach to Rayleigh streaming. Debbie Sastrapradja and Victor W. Sparrow (Grad. Prog. in Acoust., Penn State, 217 Appl. Sci. Bldg., University Park, PA 16802)

In 1995 Kamakura et al. [J. Acoust. Soc. Am. 97 (5, Pt. 1), 27402746] showed how an incompressible, viscous fluid stream-function vorticity formulation could be applied to model the time dependent build up of acoustic streaming in and around the focal area of an ultrasonic transducer in water. They found excellent qualitative agreement between their numerical results and experimental findings available in the literature. The purpose of the present research is to apply this numerical method to the Rayleigh streaming encountered in resonator tubes of thermoacoustic devices. A new code has been written from scratch and benchmarked against the published results of Kamakura et al. with excellent agreement. An overview of both the algorithm and application method to the Rayleigh streaming problem briefly will be provided. Initial results and comparisons to recent analytical solutions will be given. While not as flexible as highresolution computational fluid dynamics calculations on large parallel computers for the study of acoustic streaming, the present formulation is much faster and can run on a desktop machine. [Work supported by ONR.] 
2aPA8. Acoustic streaming generated by standing waves in narrow channels. Mark F. Hamilton, Yurii A. Ilinskii, and Evgenia A. Zabolotskaya (Dept. of Mech. Eng., Univ. of Texas, Austin, TX 78712-1063)

Considerable attention has been devoted to acoustic streaming following the initial investigation by Rayleigh. A standing wave in a channel generates acoustic streaming in the form of inner and outer vortices that circulate in opposite directions. The outer vortex is the streaming pattern described by Rayleigh. The inner vortex is generated within the viscous boundary layer and has comparable thickness. Because boundary layer thickness is typically an exceedingly small fraction of channel width, the inner vortex is normally disregarded in relation to the Rayleigh streaming. However, channel widths in stacks used in thermoacoustic engines are comparable to boundary layer thickness. As channel width decreases, the relative thickness, of the inner vortex increases as that of the outer vortex decreases, and when channel width is comparable to the boundary layer thickness, the inner vortex dominates the flow field. Previous studies of inner streaming vortices have focused mainly on the problem of a cylinder oscillating in an infinite fluid. Acoustic streaming in narrow channels is studied here analytically and numerically. An analytical solution is derived in the quadratic approximation. Fully nonlinear numerical results are obtained from a 2-D code recently developed for thermoacoustic engines.
11:00

2aPA9. Numerical study of resonant gas oscillation in a closed tube with varying cross section. Takeru Yano (Dept. of Mech. Sci., Hokkaido Univ., Sapporo 060-8628, Japan, yano@mech-me.eng.hokudai.ac.jp)

Nonlinear resonant gas oscillations in closed tubes with varying crosssections filled with an ideal gas are numerically studied. The resonant gas oscillation concerned is excited by a piston at one end of the tube, which oscillates harmonically with a resonant frequency obtained by solving the quasi-1-D linear wave equation. The system of Navier-Stokes equations for axisymmetric flow is directly solved by a high-resolution upwind TVD finite-difference scheme. The numerical results may be summarized as follows: (i) If the variation of cross-section is small, a quasi-steady oscillation state includes shock waves, as in the tubes of uniform cross-section. (ii) When the variation of cross-section is relatively large, the gas oscillation evolves into a standing wave of large amplitude, but shock waves are not formed. (iii) The (nondimensional) maximum amplitude can attain the order of the cubic root of M, where M is the acoustic Mach number at the sound source. (iv) Such a large amplitude oscillation induces relatively fast acoustic streaming.

\title{
Session 2aSA
}

\section{Structural Acoustics and Vibration: Methods of Passive Dissipation in Structural Acoustics I}

\author{
George Lesieutre, Chair \\ Department of Aerospace Engineering, Pennsylvania State University, State College, Pennsylvania 16802
}

Invited Papers

9:00

2aSA1. Passive damping applications for noise and vibration reduction. Conor D. Johnson, Roger M. Glaese, and Paul C. Janzen (CSA Eng., Inc., 2565 Leghorn St., Mountain View, CA 94043)

This paper presents an overview of several unique techniques and their applications for passive damping for vibration and noise control. Most of the methods that are used target structural vibration and by its attenuation, transmitted acoustics as well. Viscoelastic materials are the most widely used technology for applications. An innovative family of viscoelastic materials, having fairly constant modulus and loss factor properties with respect to temperature, has recently been developed, with some materials in this family having very good thermal conductive properties as well. Another innovative viscoelastic method involves co-curing the viscoelastic and a composite host structure. Additionally, an innovative acoustic enclosure for precision manufacturing has been developed that relies on barrier mass and structural damping to attenuate acoustic transmission. Passive magnetic damping has been used in both tuned mass dampers and passive isolation systems for structural-acoustic systems. Research has been conducted on the design and applications of particle damping. By placing a number of small particles in a cavity of a vibrating structure, passive damping may be achieved over wide frequency and temperature ranges. The use of all of these methods will be illustrated through ground, air, and space-based examples.

\section{9:30}

2aSA2. A method for increasing the damping properties of fiber reinforced composite structures. Anthony A. Caiazzo and B. Walter Rosen (Mater. Sci. Corp., 500 Office Ctr. Dr., Ste. 250, Fort Washington, PA 19034)

The passive material damping concept discussed in this paper is a sandwich construction of continuous fiber reinforced composite layers separated by a constrained visco-elastic material (VEM) layer. The fiber orientations in the composite layers are spatially oriented to produce systematic stiffness variations. It has been shown that three independent shear components may be developed in the VEM for simple applied loads using this spatially varying stiffness (SVS) approach, which is a distinct improvement over other traditional constrained layer treatments. To demonstrate predicted high-damping levels, modal loss factor data are presented for simple tubular and beam bending elements. Results of airborne sound transmission loss measurements on 1-m square SVS panels of various areal densities are described. Random vibration levels for an equipment mounting plate constructed using the SVS material are also presented and compared to results for a metallic base plate. 
2aSA3. Analytical/experimental studies of integral passive damping in composite isogrid structures. Ronald Gibson and Yu Chen (Mech. Eng. Dept., Wayne State Univ., Detroit, MI 48202)

Composite isogrid structures are currently in use in a number of aerospace applications where the control of structural vibration response is an important design consideration. In this presentation, a recent investigation of the modal vibration characteristics of composite isogrid structures with embedded viscoelastic damping layers will be summarized. Analysis was done by means of a finite element method implementation of the modal strain energy method, while experiments were done by using an impulse-frequency response technique. Composite isogrid test panels were fabricated by a fast and inexpensive thermoplastic stamping process using two different co-mingled materials: glass/polypropylene and carbon/nylon. The damping materials were embedded at the interface between the ribs and the skin where the shear deformations are greatest in order to significantly improve the damping properties of the structures. It is found that the required amount of damping material can be reduced significantly if it is optimally placed. The modal vibration characteristics of composite isogrid structures are also compared with those of other composite structures such as laminates and sandwich panels. The authors gratefully acknowledge the support of the WSU Institute for Manufacturing Research, as well as donations of materials by Vetrotex America, Cytec Fiberite, and 3M Company.

\section{0:30-10:45 Break}

\section{Contributed Papers}

\section{0:45}

2aSA4. Damping measurements of highly damped wavy composite structures. Scott D. Sommerfeldt (Dept. of Phys., Brigham Young Univ., Provo, UT 84602), William F. Pratt, and Matthew S. Allen (Patterned Fiber Composites, Inc., Lindon, UT 84042)

Wavy composite material is a composite that has been developed in recent years that uses opposing sinusoidal fiber waveforms in adjacent layers in a manner that enhances the constrained layer damping effect in the material. Analysis of the material has predicted both high damping and stiffness, which is contrary to many materials where increased damping generally leads to decreased stiffness of the material. To validate the analysis, a test system has been developed to measure both the damping and the stiffness properties of the material under axial excitation. The system has been shown to be capable of measuring frequency dependent damping coefficients of less than $1 \%$. Tests on the wavy composite have led to a characterization of the material over a broad range of frequency and temperature that agrees well with predicted values, and allows one to design the wavy composite lay-up to give a desired performance at a given temperature and frequency. Damping measurements as high as 30\% have been measured, while maintaining stiffness properties that are comparable to materials such as aluminum and titanium, using standard graphite fibers.

\section{1:00}

2aSA5. Forced response condensations applied to the eigenvalue analysis of damped structures. Zhao Xianfeng and J. Gregory McDaniel (Dept. of Aerosp. and Mech. Eng., Boston Univ., 110 Cummington St., Boston, MA 02215)

The eigenvalue analysis of nonproportionally damped structures has received much recent attention, as most of the algorithms to solve undamped problems do not directly extend to the damped case. In many cases, one is only interested in finding the eigenvalues that lie in a region of the complex plane. In this presentation, we propose an algorithm that exploits this fact. The algorithm begins by finding the eigenvalues of the undamped problem and tracks the eigenvalues as damping is gradually added. For each increment of damping, new eigenvalue estimates are obtained by forcing the structure at the previous eigenpair and using the forced response vectors to condense the matrices. In this way, one may be assured of finding all of the eigenvalues in a vertical strip of the complex plane. Features of the algorithm will be discussed and initial proof-ofconcept results will be presented. [This material is based upon work supported by NSF under Grants Nos. 9978747 and 9984994.$]$

\section{$11: 15$}

2aSA6. A new type of vibration damper based on flexural wave propagation in laminated wedges of power-law profile. Victor V. Krylov (Dept. of Aeronautical and Automotive Eng., Loughborough Univ., Loughborough, Leicestershire LE11 3TU, UK, V.V.Krylov@lboro.ac.uk)

Flexural waves propagating towards sharp edges of elastic wedges are slowing down and growing in amplitudes as they approach the edges. Especially interesting phenomena may take place in wedges having cross sections described by a power-law relationship between their local thickness and the distance from the edge, with $m$ being a power-law exponent. In particular, the theory shows that, for $m$ equal or larger than 2-in free wedges, and 5/3-in immersed wedges, the incident flexural waves can become trapped near the edge and never reflect back, i.e., the above structures represent acoustic "black holes" for flexural waves. Because of the deviations of real manufactured wedges from the ideal power-law profiles, first of all because of ever-present edge truncations, the corresponding reflection coefficients are always far from zero. Therefore, in reality such homogeneous wedges can not be used as effective vibration dampers. In the present paper it is demonstrated that the situation can be significantly improved by depositing an absorbing thin layer on the wedge surfaces. In this case the reflection coefficients can be drastically reduced even for wedges with noticeable truncations. Thus, the combination of the powerlaw profile and a thin absorbing layer can result in a new effective method of damping flexural vibrations. 
Session 2aSC

\title{
Speech Communication: Segmental Acoustics, Perception and Production (Poster Session)
}

\author{
Betty H. Tuller, Chair \\ Complex Systems and Brain Sciences, Florida Atlantic University, Boca Raton, Florida 33431
}

Contributed Papers

\begin{abstract}
All posters will be on display from 8:30 a.m. to 12:00 noon. To allow contributors an opportunity to see other posters, contributors of odd-numbered papers will be at their posters from 8:30 a.m. to 10:15 a.m. and contributors of even-numbered papers will be at their posters from 10:15 a.m. to 12:00 noon.
\end{abstract}

2aSC1. An acoustical study on Lai vowel length. Kenneth Vanbik and Rungpat Roengpitya (Dept. of Linguist, 1203 Dwinelle Hall, Univ. of California at Berkeley, Berkeley, CA 94720)

Lai (Chin), a language from the Sino-Tibetan language family, has a contrastive vowel length. In this paper, the relationship between the vowel quantity and quality in Lai and the relative duration of these vowels and the following consonant were studied. The speech of an adult male native speaker of Lai, uttering in a frame sentence words having the shape /pVC/ where $\mathrm{V}$ was long and short versions of the five phonemic vowel qualities and $\mathrm{C}$ was either $/ \mathrm{t} / \mathrm{or} / \mathrm{n} /$, was digitized. The $F 1, F 2$, and the duration of the vowels were measured as well as the duration of the final nasal. The data revealed that the long:short vowel duration ratio was about 2.5 for the tokens with a final stop and about 2.86 for the tokens with a final nasal. Vowels preceding a stop tended to be overall shorter than vowels preceding a nasal; final nasals after short vowels were significantly longer than after long vowels; short vowels were more centralized than long vowels. Thus although the vowels described as long:short are longer versus shorter, there are other correlated differences serving this contrast.

2aSC2. Acoustic characteristics of fricatives in Arabic. Mohamed Al-Khairy and Ratree Wayland (Prog. in Linguist., 4131 Turlington Hall, P.O. Box 115454, Univ. of Florida, Gainesville, FL 32611-5454)

Previous work on English fricatives suggests that a conglomerate of acoustic cues exist to differentiate among fricatives produced at different places of articulation [e.g., Jongman (2000)]. These include spectral peak location, spectral moments, $F 2$ onset frequency, noise amplitude, noise duration, etc. The goal of this study is to investigate whether or not acoustic characteristics served to classify English fricatives place of articulation can be used to classify fricatives place of articulation in Makkan Arabic. Unlike English, this dialect of Arabic includes velar, pharyngeal, and glottal fricatives in its phonological inventory. Thus of great interest is whether or not cues to fricatives place of articulation thus far discovered will be able to successfully differentiate among these rare fricatives. All acoustic measurements found in Jongman's 2000 work including locus equations will be used to explore this important question, and results of this study will be compared and contrasted with those of earlier studies.

2aSC3. Effects of consonant aspiration on Mandarin tones. Ching X. $\mathrm{Xu}$ and $\mathrm{Yi} \mathrm{Xu}$ (Dept. of Commun. Sci. and Disord., Northwestern Univ., Evanston, IL 60208, xxq@northwestern.edu)

In this paper we present an experiment on the influence of consonant aspiration in Mandarin. A minimal pair, /da/ and /ta/, which differ only in aspiration, are produced in carrier sentences by native speakers of Mandarin. It is found that consonant aspiration affects the fundamental frequency $(F 0)$ of the following vowel. The onset $F 0$ of a tone is higher following unaspirated consonants than following aspirated consonants, and the magnitude of difference is related to both the current and preceding tones. However, the later part of a tone tends to be lower following an unaspirated consonant than an aspirated one. These results seem to favor the speech aerodynamic model proposed by Ohala (1976), which predicts a smaller value of airflow at the onset of the vowel following an aspirated stop than an unaspirated one. The current results, however, seem to provide further details about the mechanism of the aerodynamic effect. Additionally, results of detailed analyses seem to suggest a possible source of the existing controversies over the exact effect of aspiration on $F 0$. The discrepancy in the previously reported effects may be partially due to the difference in how the first few vocal cycles of the vowel are measured in different studies.

2aSC4. Tonal assimilation in Thai. Rungpat Roengpitya and John Ohala (Dept. of Linguist., Univ. of California, Berkeley, CA 94720)

Thai has five distinctive tones: mid, low, rising-falling, high, and falling-rising. In this paper, the behavior of Thai tones when they were concatenated with other tones was studied. The target utterances were Thai meaningful and nonsense words with the shape $\mathrm{C} 1 \mathrm{VC} 2$, where the $\mathrm{C} 1$ was a nasal, the $\mathrm{V}$ was /i/ or /i:/, bearing each of the five tones, and $\mathrm{C} 2$ was either a nasal or a voiceless stop. The targeted words were spoken by three native speakers of Thai in isolation and in five different tonal contexts, digitally recorded, and measured for the fundamental frequency of the targeted words and the following words. The results showed that the tones of the targeted words normally started at the vowel onset but their offset carried over to the beginnings of the tones of the following words. This carryover effect [previously noted by Xu (1997) for Mandarin tones] occurred more for the targeted words with the final nasals than for the ones with the final stops.

2aSC5. Variation of reaction times for the recognition of voiced plosives with preceding noise duration. William Ainsworth (MacKay Inst. of Commun. and Neurosci., Keele Univ., Staffs ST5 5BG, UK)

Voiced plosives in noise which precedes the speech are recognized correctly more often than in noise which begins at the start of the speech. This effect may be due to noise adaptation of firing thresholds of neurones in the cochlear nerve effectively performing spectral subtraction, or it may be due to perceptual streaming at a higher level of the auditory system. In order to investigate this further, reaction times for recognition have been measured. It was found that the reaction time initially reduces for a preceding noise of $100-200 \mathrm{~ms}$, then increases as the noise duration is lengthened (at least up to $800 \mathrm{~ms}$ ). Furthermore it was found that the reaction time is reduced when the recognition score is higher. Assuming that recognition consists of processing which increases with the noise 
duration but decreases for good internal matches, a model of reaction time as a function of preceding noise duration has been developed. It consists of two negative exponential functions: one a function of noise duration and the other a function of recognition score. Good agreement between experimental data and the model has been found. [Work supported by EU TMR Contact No. FMRX-CT97-0150.]

2aSC6. Phonological and acoustic-phonetic cues in recognizing words ending in nonrelease stop consonants. Janielle Lugo-Marin and Cynthia M. Connine (Dept. of Psych., State Univ. of New York, Binghamton, NY 13902)

This study investigates the effects of probabilistic likelihood and of certain acoustic-phonetic cues in the recognition of word-final nonrelease stop consonant stimuli. Recognition of the word was examined by assessing the activation of an embedded word (inconsistent with a release) relative to the activation of the whole word. The missing release was presented in contexts that predict a higher (a following consonant) or lower (a following vowel) likelihood of a release. The contribution of acousticphonetic cues was examined by including word-final stop consonants that were voiced (do not require release for recognition) or voiceless (release required for recognition). Four experiments were designed to examine the effects of the probabilistic likelihood for the release and of acousticphonetic cues in the activation of a canonical version of a word, when the final stop-consonant release is not present. Priming of the embedded word was greater for the voiceless relative to the voiced stimuli, and also for the following consonant context relative to vowel context. In the absence of the following context, the embedded word was primed for the voiceless but not for the voiced stimuli, and the whole word was primed to a greater extent for the voiced relative to the voiceless stimuli.

2aSC7. Relative timing of oral and laryngeal motions in voiceless consonant production: A comparison of single and geminate consonants in Japanese. Akinori Fujino (NTT Commun. Science Labs., NTT Corp., 3-1 Morinosato Wakamiya, Atsugi-shi, Kanagawa 243-0198, Japan, fujino@idea.brl.ntt.co.jp), Tokihiko Kaburagi (Dept. of Acoust. Design, Kyushu Inst. of Design, Minami-ku, Fukuoka 815-8540, Japan), Masaaki Honda (NTT Commun. Sci. Labs., NTT Corp., Atsugi-shi, Kanagawa 243-0198, Japan), Emi Z. Murano (Dept. of Otolaryngol., Univ. of Tokyo, Bunkyo-ku, Tokyo 113-0033, Japan), and Seiji Niimi (Dept. of Speech-Lang. Pathol. and Audiol., Intl. Univ. of Health and Welfare, Otawara-shi, Tochigi 324-0011, Japan)

It is known that temporal coordination of oral and laryngeal articulations is important in voiceless consonant production. In this study, the relative timing of oral constriction and glottal opening is investigated for single and geminate voiceless consonants in Japanese. The motions of the jaw, lips, tongue, and larynx of three male speakers were examined using EMA (electromagnetic articulograph), PGG (photoglottograph), and EGG (electroglottograph) techniques. A set of /iie eCe desu/ and /iie eQCe desu/ sentences, containing voiceless consonants $/ \mathrm{s}, \mathrm{t}, \phi, \mathrm{p} /$, was used for the test utterances. /QC/ in the sentence shows a geminate form in Japanese. The experiment data showed that the interval between the peak of glottal opening and oral release onset for geminates was longer than for singles and that consonant duration was also longer. For each form, the longer the consonant duration was, the longer the interval became. These temporal relations were found for all speakers, although the ratio of the two intervals was different for each speaker. The results suggest that, for both single and geminate consonants, articulators are controlled based on the consonant duration dependence of the oral-glottal timing. [Work supported by CREST, JST.]
2aSC8. Transconsonantal coarticulatory patterns in VCV utterances: Effects of a bite block. Yana Yunusova and Gary Weismer (Waisman Ctr. and Dept. of Communicative Disord., Univ. of Wisconsin, Madison, Madison, WI 53705-2280)

The concept of coordinative structures, or articulatory synergies, envisions a collection of articulators organized to achieve a specific articulatorly or acoustic goal. The mandible figures prominently in concepts of articulatory synergies because of its potential interaction with labial and lingual shaping of the vocal tract. What happens to these hypothesized synergies when one component is taken out of the collective? Bite block articulation is a common experimental approach to eliminating the jaw from its synergistic role in articulation, and studies have shown that the speech mechanism is able to reorganize its target configurations almost immediately when speaking with a fixed jaw. In the current study we examine vowel-to-vowel, transconsonantal effects with and without a bite block. Although there is one study [Sussman, Fruchter, and Cable, J. Acoust. Soc. Am. (1995)] showing stability of coarticulatory effects in biteblock conditions, that conclusion was based primarily on locus equations. In the current study, we hypothesize that more traditional acoustic measures of right-to-left and left-to-right coarticulation will show that reducing an articulatory synergy by holding one of its components constant will result in different-from-typical coarticulatory behaviors. [Work supported by NIDCD Award No. DC 000319.]

2aSC9. A cross-linguistic study of articulatory timing in liquids. Bryan Gick, Fiona Campbell, and Sunyoung Oh (Dept. of Linguist., Univ. of British Columbia, E270-1866 Main Mall, Vancouver, BC V6T 1Z1, Canada, gick@interchange.ubc.ca)

A number of recent studies have observed that the timing of articulatory gestures is systematically different in prevocalic versus postvocalic allophones of English liquids. In English, the more anterior (consonantlike) gestures tend to occur at the peripheries of syllables, while more posterior (vowel-like) gestures occur nearer the syllable peak. These facts have allowed for a greatly simplified account of phonological processes involving English liquids, such as "vocalization" of postvocalic liquids, $r$ or 1-coloring of vowels, and "dark" and "light" allophones of /1/. However, no such studies have been made of timing in liquids of other languages. In this study, ultrasound data were collected from one to three subjects for each of English /1/ and /r/, Quebecois French /l/, Mandarin /r/, and Korean /1/. Differences in intergestural timing were measured for prevocalic versus postvocalic allophones. Results show similar timing effects to those previously observed for English, with posterior gestures occurring earlier in postvocalic allophones. This property is consistent with phonological processes, such as pre-liquid laxing, observable in these languages. Quebecois /1/ and Mandarin/r/ were also interesting in exhibiting no measurable dorsal gesture at all in their initial allophones. [Research supported by the NSERC.]

2aSC10. MRI motion imaging of articulatory movement-coarticulation effects in velar consonants. Noriko Suzuki (School of Dentistry, Showa Univ., 2-1-1 Kitasenzoku, Ohta-ku, Tokyo, Japan), Tatsuya Kitamura (Faculty of Information, Shizuoka Univ., 3-5-1 Johoku, Hamamatsu, Shizuoka, Japan), Shinobu Masaki (ATR Human Information Processing Res. Labs.), and Ken-ichi Michi (School of Dentistry, Showa Univ., 2-1-1 Kitasenzoku, Ohta-ku, Tokyo, Japan)

Magnetic resonance imaging (MRI) techniques have been used in speech research for measuring the vocal tract. Recently, MRI motion imaging has been developed and applied to the study of speech production. In this study, the coarticulatory effects of Japanese velar stop consonants were measured using a synchronized sampling method. The MRI technique employed is a synchronized sampling technique which is a modification of one originally developed for cardiac motion imaging. Speech samples consisted of /aka/ and /aki/, including the Japanese velar stop consonant / $/ \mathrm{k}$ and vowel /i/, in normal subjects. Results depicted motion of the speech organs in the midsagittal plane. When velar consonants were 
followed by a back vowel /a/, the back of the tongue contacted the posterior part of the soft palate, and when followed by front vowel /i/, tongue contact was more toward the anterior of the soft palate. Thus the tongue contact position for Japanese velar stop consonants was found to be strongly influenced by the following vowel.

2aSC11. Phase transitions in a repetitive speech task as gestural recomposition. Kenneth J. de Jong, Byung-jin Lim, and Kyoko Nagao (Dept. of Linguist., Indiana Univ., 322 Memorial Hall, Bloomington, IN 47405, kdejong@indiana.edu)

Tuller and Kelso (JSHR, 1991) show the relative timing of peak glottal opening and oral closure in repetitive /ip/ production differs between /ip/ and /pi/ productions. Also, as repetition rate increases, the timing in /ip/ merges with that in /pi/. The authors interpret this to indicate two stable regions in glottal-to-oral phasing, one for codas and one for onsets. The current paper examines a similar corpus of glottal transillumination traces of repeated syllables, which includes both labial and lingual stops. Preliminary examination of peak glottal timing with respect to acoustic landmarks indicating closure shows the same pattern of timing differences at slow rates and neutralization at fast rates. However, peaks in the transillumination traces often do not indicate glottal abduction for the consonant, but rather indicate a relatively open glottis for vocalic voicing, happening in the context of glottal stoppage at the onset of the vowel. In addition, coronal codas exhibit cycle-to-cycle variation from glottalized to abducted voiceless forms. Thus while rate does induce stepwise changes in glottal activity, phasing, in the sense of relative timing, does not account for many of the rate effects. Rather, rate appears to be inducing a recomposition of the gestures which comprise the speech.

2aSC12. Gestural timing effects in the "perfect memory" sequence observed under three rates by electromagnetometry. Mark K. Tiede (M.I.T.-R.L.E., Cambridge, MA and Haskins Labs, 270 Crown St., New Haven, CT 06520, tiede@ @askins.yale.edu), Joseph Perkell, Majid Zandipour (M.I.T.-R.L.E., Cambridge, MA), and Melanie Matthies (Hearing Res. Ctr., Boston Univ. and M.I.T.-R.L.E., Cambridge, MA)

In a well-known example due to Browman and Goldstein [Lab. Phon. I, 341-376 (1990)] the / $\mathrm{ktm} /$ sequence in the phrase "perfect memory" is contrasted between careful (list) and fluent production conditions. X-ray microbeam data were used to show that although in the fluent case coproduction of the $/ \mathrm{m} /$ can mask the acoustic releases of the $/ \mathrm{k} /$ and $/ \mathrm{t} /$, both stops are nonetheless articulated. The current work uses EMMA data to examine this sequence in greater detail: 8 subjects produced the phrase in a carrier context under normal, fast, and clear rate conditions. Results confirm that tongue dorsum and tip movements toward velar and apical closure occur regardless of rate and observable acoustic effect. In addition, while movement amplitudes decreased somewhat as rate increased, little variation in the durations associated with the consonant gestures was observed. Instead, changes in rate primarily effected $\mathrm{V}$ to $\mathrm{V}$ duration and the relative phasing of the velar and apical closing gestures: the tongue tip maximum $(/ \mathrm{t} /)$ lagged that of the tongue dorsum $(/ \mathrm{k} /)$ in clear speech, was aligned with it in normal speech, and generally anticipated it in the fast rate condition. [Work supported by NIH.]

2aSC13. Correlations between vowel and the subsegments of an obstruent in CV and VCV sequences. Hansang Park (Dept. of Linguist., Calhoun Hall, Univ. of Texas, Austin, TX 78712)

It is well known that there is a significant negative correlation between a consonant and the preceding or following vowel. However, it has seldom been specified what relationship lies between the subsegments (closure, frication, and aspiration) of an obstruent and the preceding or following vowel in Korean. This work investigates the relationship between the vowel and the subsegments of a Korean coronal obstruent in CV and VCV sequences. There was a significant negative correlation between the gap and vowel $(r=-0.900)$ in utterance initial CV sequence but no significant correlation between frication and vowel. On the other hand, there was a significant negative correlation between the preceding vowel and closure ( $r=-0.510$ ) in the VCV sequence but no significant correlation between the preceding vowel and other subsegments. There was a significant negative correlation between the following vowel and gap $(r=-0.545)$ but no significant correlation between the following vowel and other subsegments. Generally, there was a significant correlation between the vowel and an immediately adjacent segment than between the vowel and remote segments. Correlation was stronger in the CV sequence than in the VCV sequence for the same pair of variables. Speech Communication Best Student Paper Award.

2aSC14. Dynamic center-of-gravity effects in consonant-vowel transitions. Ewa Jacewicz, Nandini Iyer, Robert A. Fox, and Lawrence L. Feth (The Ohio State Univ., 1070 Carmack Rd., Columbus, OH 43210)

Lublinskaja has reported that dynamic changes in the spectral centerof-gravity (COG) of one formant in selected Russian vowels led to changes in the identification of the vowel. Movement of the COG was effected by simultaneous amplitude modulation of two formants placed at the end points of the desired frequency transition [Iyer et al., J. Acoust. Soc. Am. 109, 2294 (2001)]. The present study explores whether such COG effects will extend into the processing of consonant-vowel transitions (CVs). A [da]-[ga] continuum was generated using a Klatt synthesizer with a 50-ms CV transition and a $200-\mathrm{ms}$ vowel. The onset of $F 3$ varied from $2018-2818 \mathrm{~Hz}$ in $80-\mathrm{Hz}$ step. The $F 3$ of the vowel remained constant at $2527 \mathrm{~Hz}$. Three different stimulus sets differed in terms of whether the $F 3$ transition was a: (1) formant, (2) FM tone, or (3) virtual frequency (VF) glide. In the VF stimuli, the frequencies of two tones matched the onset or offset frequencies of the $F 3$ transition and modulation of their amplitudes produced a gliding spectral COG. Preliminary results indicate that listener performance is not strongly affected by changing the means by which spectral changes to $F 3$ are made. [Research supported by a grant from OSU's College of Social and Behavioral Sciences to L. Feth and an INRS award from NIH to R. Fox.]

2aSC15. Perceptual and articulatory noise in vowel imitation. Gautam K. Vallabha and Betty Tuller (Ctr. for Complex Systems and Brain Sci., Florida Atlantic Univ., 777 Glades Rd., Boca Raton, FL 33431)

Speakers exhibit systematic errors when asked to imitate self-produced vowels [B. Tuller and G. Vallabha, J. Acoust. Soc. Am. 107, 2855 (2000)]. These results were replicated ( 3 speakers, 45 systematically distributed self-produced vowel targets, 10 imitations per target) and compared to a simulation of the effects of articulatory and perceptual noise. Articulatory noise was modeled using the Haskins Articulatory Synthesizer. The tongue body center (TBC) was interpolated between the configurations for /i/, /a/, and $/ \mathrm{u} /$ and 90 of the intermediate configurations were perturbed by adding independent Gaussian noise ( $\mathrm{sd}=1 \mathrm{~mm}$ ) to the $x$ and $y$ coordinates of the TBC. Perceptual noise was modeled by (1) transforming each of the 90 intermediate vowels to $F 1 / F 2$ bark ("perceptual") space and (2) adding independent Gaussian noise ( $\mathrm{sd}=$ vowel discrimination limen $=0.28$ barks) to each bark coordinate. The models did not match the directions of the imitation errors made by the speakers. However, the distribution of the error magnitudes, relative to the mean imitation, was approximately Rayleigh (0.28) across both the models and the speakers. These results indicate that while speech perception and/or production may have intrinsic Gaussian noise, this noise is not responsible for the systematicity of the imitation errors. [Work supported by NIMH.] 
2aSC16. Vowel formant discrimination in quiet, speech-shaped noise, and babble for natural speech. Chang Liu and Diane Kewley-Port (Dept. of Speech and Hearing Sci., Indiana Univ., Bloomington, IN 47405)

Vowel formant discrimination in quiet and in noise for naturally spoken vowels was investigated. The stimuli were seven isolated vowels $/ \mathrm{i}, \mathrm{u}$, I, $\varepsilon, \Lambda, æ, a /$ recorded from a female talker and resynthesized using STRAIGHT with and without formant shifts. Vowels were presented in two noise types, long-term speech-shaped noise (LTSSN) and twelvetalker babble. $\Delta F$ was measured for $F 1$ and $F 2$ in quiet and noise with five signal to noise ratios (SNRs), $+4,+2,0,-2$, and $-4 \mathrm{~dB}$. Adaptive tracking procedures were used, interleaving quiet and SNR levels for one formant at a time. Overall, results suggested that formant frequency, SNR, and noise type had significant effects on $\Delta F$. For either LTSSN or babble, $\Delta F$ as a function of formant frequency showed four significantly different patterns, depending on the SNRs. $\Delta F$ as a function of SNR also showed several different patterns for different formants. A surprising result was that $\Delta F s$ were higher at $-2 \mathrm{~dB}$ than those at $-4 \mathrm{~dB}$ in babble, particularly in the $F 2$ region. In addition, performance in babble was significantly better than that in LTSSN at comparable SNRs. These results will be examined by partial loudness models. [Work supported by NIHDCD02229.]

2aSC17. Effects of synthesis fidelity on vowel identification: Role of spectral change and voicing source. Peter F. Assmann and William F. Katz (School of Human Development and Callier Ctr. for Community Disord., The Univ. of Texas at Dallas, Box 830688, Richardson, TX 75083)

Recent studies have shown that synthesized versions of American English vowels are more accurately identified when natural time-varying changes in the formant frequencies are preserved rather than flattened. A limitation of these experiments is that vowels generated with cascade formant synthesis are generally less accurately identified than natural vowels. To overcome this limitation, a high-quality analysis-synthesis system was used to reexamine the effects of spectral change. Using this new technique, synthesized versions of 12 American English vowels spoken by adults and children were identified as accurately as natural vowels. Two experiments confirmed the beneficial effects of preserving the timevarying changes in the formants, both in vowels synthesized with pulsed excitation (as in voiced speech) and noise excitation (as in whispered speech), and verified that identification accuracy does not decline when the fundamental frequency is held constant. However, in contrast to earlier findings, (i) whispered vowels were identified as accurately as the voiced versions, and (ii) the benefits of time-varying spectral change were greater than previously found with cascade formant synthesis. The findings are consistent with recent studies showing that the measured effects of spectral and temporal manipulations in vowels can vary as a function of synthesis fidelity.

2aSC18. Recognizing vowels from narrow band spectra. James $M$. Hillenbrand (Speech Pathol. and Audiol., Western Michigan Univ., Kalamazoo, MI 49008) and Robert A. Houde (RIT Res. Corp., Rochester, NY 14623)

Most models of vowel recognition begin with a representation consisting of either a smooth envelope spectrum or formant frequencies extracted from those envelope spectra. In the present report we describe a classification method that performs pattern matching by comparing highresolution vowel spectra (i.e., spectra that retain harmonics of the voice source) with a set of smoothed spectral-shape templates for each of the vowels derived by averaging harmonic spectra from a large database of $/ \mathrm{hVd} /$ utterances spoken by men, women, and children [Hillenbrand et al., J. Acoust. Soc. Am. 97, 3099-3111 (1995)]. [Work supported by NIH.]
2aSC19. Intelligibility of narrow-band speech is enhanced by unmodulated flanking noise: Restoring intelligibility by masking of silence. Peter W. Lenz, James A. Bashford, Jr., and Richard M. Warren (Dept. of Psych., Univ. of Wisconsin-Milwaukee, Milwaukee, WI 53201, plenz@uwm.edu)

The intelligibility of notch-filtered sentences may improve by $10 \%$ or more when a large spectral gap is filled with noise rather than silence [Warren et al., P\&P 59, 275-283 (1997)]. The present study shows that a comparable "spectral completion" (SC) effect occurs when a narrow band of speech is surrounded by complementary noise. In Experiment 1, intelligibility of $2 / 3$-octave everyday sentences, centered at $1.5 \mathrm{kHz}$ [2000order FIR filtering, slopes greater than $1000 \mathrm{~dB} /$ octave], was $65 \%$ without noise, $72 \%$ with complementary low-pass or high-pass noise, and $76 \%$ with both noise bands present (noise spectrum level at $20 \mathrm{~dB}$ relative to the speech). Experiment 2 found a comparable SC effect with partially masked speech: The 2/3-octave sentences were about $40 \%$ intelligible when masked by narrow-band noise having the same spectral limits (relative spectrum level again $20 \mathrm{~dB}$ ), but were $50 \%$ intelligible when the noise was broadband. Hence, out-of-band noise enables listeners to make better use of information within narrow-band speech, perhaps by masking an otherwise misleading absence of energy in normally coactive regions of the spectrum. Under partial masking conditions, this SC effect can produce an apparent release from masking in the absence of comodulation. [Work supported by NIH.]

2aSC20. Dual band speech intelligibility: Implications of some novel observations concerning amplitude mismatch and asynchrony. Richard M. Warren, James A. Bashford, Jr., and Peter W. Lenz (Dept. of Psych., Univ. of Wisconsin-Milwaukee, Milwaukee, WI 53201, rmwarren@uwm.edu)

Two 1/3-octave bands of everyday sentences (center frequencies 1 and $3 \mathrm{kHz}$ ) were employed. To avoid the major contribution of slopes (even when $100 \mathrm{~dB} /$ octave) upon intelligibility [Warren and Bashford, J. Acoust. Soc. Am. 106, L47 (1999); Warren et al., J. Acoust. Soc. Am. 108, 1264 (2000)], nearly vertical slopes were employed (4000 order FIR filtering producing $2000 \mathrm{~dB}$ /octave slopes). Heard alone at $75 \mathrm{~dB}$, intelligibilities were $5 \%(1 \mathrm{kHz})$ and $10 \%(3 \mathrm{kHz})$; together, the synergistic score was $80 \%$. When the $3-\mathrm{kHz}$ band was kept at $75 \mathrm{~dB}$ and the $1-\mathrm{kHz}$ band's amplitude was decreased systematically, intelligibility remained unchanged (1.4\%) from 75 to $45 \mathrm{~dB}$ (amplitude ratio of 1000:1). But when both bands were at their normal relative levels, and one band was delayed relative to the other, intelligibility dropped to half with asynchrony of only about $35 \mathrm{~ms}$ (approximately half the duration of the average phoneme), decreasing to an intelligibility score of a single band when asynchrony approximated phonemic duration. It is suggested that broadband speech processing relies upon cross-frequency comparison of dynamic changes in local patterns with little regard to differences in pattern amplitude. This comparison appears to be based upon units approximating the duration of the average phoneme. [Work supported by NIH.]

2aSC21. Phonemes at the cocktail party: Insights on the processing of competing speech signals. James A. Bashford, Jr., Richard M. Warren, and Peter W. Lenz (Dept. of Psych., Univ. of Wisconsin-Milwaukee, Milwaukee, WI 53201, bashford@uwm.edu)

A recorded word repeated over and over is heard to undergo a series of illusory changes (verbal transformations) to other syllables and words in the listeners lexicon. When cocktail party listening conditions are simulated by adding a second image of the repeating word through dichotic presentation with an interaural delay preventing fusion, the resulting two distinct lateralized images of the word undergo independent transformations [Lenz et al., J. Acoust. Soc. Am. 107, 2857 (2000)]. The present study found that adding a second image of the same word increased the extent of phonetic distortions for each image, whereas presenting a different competing word reduced the transformation rate by one-half when the 
competitor differed by one phoneme (e.g., "dark" paired with contralateral "bark") and by three-fourths when it differed in all phonemes (e.g., "dark" versus contralateral "light" or "might"). These interactions were independent of semantic relations and occurred with competitors from both same and different speakers. In contrast, transformations were unaf- fected by a nonspeech competitor (a repeating contralateral door slam). These and additional results indicate that shared resources at an abstract level of phonological analysis provide the basis for the independent syllabic/lexical organizations derived for competing speech signals. [Work supported by NIH.]

\title{
Session 2aSP
}

\section{Signal Processing in Acoustics, Acoustical Oceanography, Underwater Acoustics, Biomedical Ultrasound/ Bioresponse to Vibration: Acoustic Image Reconstruction Using Tomographic Techniques}

\author{
Sean K. Lehman, Cochair \\ Lawrence Livermore Laboratory, P.O. Box 808, L-154, Livermore, California 94551 \\ Michael Silevitch, Cochair \\ Northeastern University, 360 Huntington Avenue, Room 235FR, Boston, Massachusetts 02115 \\ Chair's Introduction-8:55 \\ Invited Papers \\ 9:00 \\ 2aSP1. The Center for Subsurface Sensing and Imaging Systems (CenSSIS): Addressing the challenge of implementing a \\ strategy of "diverse problems similar solutions." Michael Silevitch (Northeastern Univ., CenSSIS, 360 Huntington Ave., Boston, \\ MA 02115, msilevit@1ynx.neu.edu) \\ CenSSIS has recently been designated by the National Science Foundation as an Engineering Research center (ERC). The \\ CenSSIS mission is to unify the methods and tools used to detect and image biomedical and environmental-civil objects or conditions \\ that are underground, underwater, or embedded in the human body. In the area of physics based signal processing and image \\ understanding, methods of multiview tomography play a key role. Beginning with a brief description of CenSSIS, this talk will then \\ focus upon a discussion of a unified framework that tries to articulate a methodology for characterizing end to end subsurface sensing \\ and imaging problems. Several examples will be used to illustrate the ideas, including ones dealing with acoustic tomography.
}

\section{9:25}

2aSP2. Acoustic wave diffraction tomography. Anthony J. Devaney (Northeastern Univ., ECE, 360 Huntington Ave., Boston, MA 02115, tonydev2@aol.com)

The classical theory of Diffraction Tomography (DT) is formulated within the context of the acoustic and elastic wave equations A number of applications for the generalized theory are reviewed including ultrasound tomography in variable density and elastic wave media and cross-well geophysical tomography and offset vertical seismic profiling (VSP). The general theory of DT is compared and contrasted with classical focus-on-transmit and focus-on-receive confocal acoustic imaging as well as with various standard ad-hoc imaging methods often employed in applications. The use of slant stacking for converting point source to plane wave insonification is reviewed as is the theory of acoustic tomography in known layered background media. A number of computer simulations and results from experimental data are presented.

$$
\text { 9:50 }
$$

2aSP3. Focusing by spatiotemporal inverse filter: Application to heterogeneous and absorbing medium. Michael Tanter, J.-F. Aubry, J.-L. Thomas, and M. Fink (Laboratoire Ondes et Acoustique, ESPCI, 10 rue Vauquelin, 75005 Paris, France, michael.tanter@espci.fr)

In most applications of ultrasound, a first step consists in focusing a broadband ultrasonic beam deeply inside the medium. Nevertheless, sound speed, density, or absorption heterogeneities may strongly degrade the focusing. Different techniques have been developed to correct such aberrations (time reversal, speckle brightness, ... .). Recently, we investigated a new broadband focusing technique, the spatiotemporal inverse filter, based on the inversion of the propagation operator relating a transducer array to a set of control points inside the medium. As this inversion is achieved both in space and time, it allows one to calculate the set of temporal signals to be emitted by each array element for an optimal focusing on a chosen control point. Potential applications have been experimentally investigated in heterogeneous and absorbing media where classical geometrical focusing is highly degraded. The strong improvement achieved by inverse filter compared to time reversal and classical focusing emphasizes the great potential of multichannel systems having the ability to apply completely different waveforms on each element. Particularly, intraplate echoes suppression in NDT, high quality focusing through a human skull, as well as hyper-resolution in reverberating media highlight its great interest in various fields such as medical imaging, nondestructive testing, room acoustics, and underwater acoustics. 


\title{
Contributed Papers
}

10:30

2aSP4. Resolution enhancement algorithm utilizing multiple reflections for image reconstruction in the conduit. Kenbu Teramoto and Kohsuke Tsuruta (Dept. of Mech. Eng., Saga Univ., Saga-shi 840-8502, Japan)

In the acoustical endscopy, due to the physical limitations, the transducer array is composed of a small number of elements and each interspacing is larger than the acoustical wave length that is called a sparse array system. In such case, the volumetric image reconstruction process becomes ill-posed caused by the interference of the reverberation in the tube. Consequently, the reconstructed image is interfered by widely spread strong spurious lobes. This paper proposes a novel volumetric image reconstruction algorithm for a 3D sparse aperture acoustical holography, which visualizes the forward-looking image in the conduit tube. The proposed algorithm utilizes prior knowledge about both field, object, and observed signal as (1) the support of the object is limited within the conduit, and (2) the multiple reflected wave field scattered by the object is statistically independent of the reverberation in the tube. The proposed sparse array acoustical imaging system utilizes, therefore, the multiple reflected wavefronts in order to enhance the resolution. By experiments, it is proven that the proposed algorithm has the following improvements: (1) Targets can be identified when multiple reflections exist in the tubes. (2) Artifacts caused by the spurious lobes can be reduced.
10:45

2aSP5. Two-dimensional synthetic focusing using large aperturesDeconvolution of the spatial impulse response. Fredrik Lingvall and Tadeusz Stepinski (Signals and Systems Group, Dept. of Mater. Sci., Uppsala Univ., P.O. Box 528, SE-751 20 Uppsala, Sweden, fredrik.lingvall@signal.uu.se)

The synthetic aperture focusing technique (SAFT) has been used successfully in both medical and nondestructive evaluation (NDE) applications of ultrasound. Transducer size is an important issue when implementing SAFT algorithms. The classical SAFT method is based on the assumption that a point source is used for emitting ultrasonic waves, which means in practice that the transducer aperture is so small compared to the wavelength that it emits spherical waves. If the transducer aperture is large its spatial impulse response (SIR) significantly differs from a Dirac pulse and nonspherical waves will be generated. Therefore, to successfully perform synthetic focusing using large apertures, the transducer's SIR must be taken into account. A new method for compensating transducer SIR in synthetic focusing is presented in the paper. The method employs a stochastic, time-domain Wiener filtering technique for two-dimensional synthetic focusing. Results of the experiments performed in water using a linear phased array are presented to demonstrate the performance of the proposed method. The use of an array enabled altering the aperture without changing the electrical characteristics of the whole system. The results obtained using the proposed technique for finite apertures are compared to those obtained with a classical SAFT algorithm.

\section{Session 2aUW}

\section{Underwater Acoustics: Seabed Characterization and Environmental Inversion}

\author{
Tracianne B. Neilsen, Chair \\ Applied Research Laboratories, University of Texas at Austin, P.O. Box 8209, Austin, Texas 78713-8029 \\ Chair's Introduction-8:25
}

Contributed Papers

8:30

2aUW1. Benchmark workshop for geoacoustic inversion techniques in range dependent waveguides. Ross Chapman (School of Earth and Ocean Sci., Univ. of Victoria, P.O. Box 3055, Victoria, BC V8W 3P6, Canada, chapman@uvic.ca), Stan Chin-Bing, David King (Naval Res. Lab., Stennis Space Ctr., MS 39529-5004), and Richard Evans (SAIC, New London, CT)

This paper summarizes the results from the ONR/SPAWAR Geoacoustic Inversion Techniques (IT) Workshop held in May 2001. The format of the workshop was a blind test to estimate unknown geoacoustic profiles by inversion of synthetic acoustic field data for vertical and horizontal array geometries in range dependent shallow water waveguides. The fields were calculated using COUPLE/RAM for three range-dependent test cases: a monotonic slope; a shelf break; and an intrusion in the sediment. Geoacoustic profiles were generated to simulate sand, silt and mud sediment environments. Different approaches for inverting the field data were presented at the workshop: model-based methods based on normal modes, parabolic equation or ray theory; perturbative methods; methods using transmission loss (TL) data; methods using vertical or horizontal array data. The geoacoustic profiles inverted by the different methods are com- pared using a metric based on calculation of transmission loss using the estimated profiles for geometries and source frequencies that were not used in the inversion. The results demonstrate the effectiveness of present day inversion techniques, and indicate the limits of their capabilities in range dependent environments. [Work supported by ONR.]

\section{8:45}

2aUW2. Geoacoustic inversion techniques workshop: Test cases 4 and 5. James K. Fulford, David B. King, and Stanley A. Chin-Bing (Naval Res. Lab., Acoust. Div., Stennis Space Center, MS 39529)

Acoustic transmission loss and reverberation data from two exercises were selected as inversion test cases for the Geoacoustic Inversion Techniques workshop, May 2001. The data was collected using air deployed sonobuoys with standard $0.82 \mathrm{~kg}$ SUS charges as sources. The acoustic data was presented in octave bands for frequencies from 25 to $800 \mathrm{~Hz}$, and a $1 / 3$ octave band for 3.5 and $6.5 \mathrm{kHz}$ center frequencies. Environmental data consisted of bathymetry, and sound speeds gathered by AXBT's. Background geophysical information was not given to the participants. Inversion results were encouraging. Selected examples will be shown. [Work supported by ONR/NRL and SPAWAR.] 
2aUW3. Inversion for source and environmental parameters using a broadband rotated coordinates technique. Tracianne B. Neilsen and David P. Knobles (Appl. Res. Lab., Univ. of Texas, P.O. Box 8029, Austin, TX 78713-8029)

The problem of localizing and tracking a source in the shallow ocean is often complicated by uncertainty in the environmental parameters. Rotated coordinates [M. D. Collins and L. Fishman, J. Acoust. Soc. Am. 98, 1637-1644 (1995)] calculated for a broadband cost function are used in simulated annealing to invert for both source and environmental parameters. The resulting broadband rotated coordinates identify the coupling and relative sensitivity the parameters over the specified bounds. Multiple sets of broadband rotated coordinates, each corresponding to a different set of bounds, are used to systematically decouple the parameters. The cost function minimized in the inversion algorithm is model-data cross-phone spectra summed coherently over phone pairs, frequency, and time sequence. The results of applying this inversion method to simulated array data are presented along with preliminary results using data from the Area Characterization Test II experiment performed on the New Jersey continental shelf. [Work supported by ONR.]

2aUW4. Matched-field inversion of seabed geoacoustic properties complemented by chirp sonar surveys. Altan Turgut and Stephen Wolf (Naval Res. Lab., Washington, DC 20375)

Matched-field inversion of range-dependent seabed parameters is studied using broadband $(525-725 \mathrm{~Hz})$ acoustic data collected on the New Jersey Shelf during the SWARM95 experiment. An efficient global optimization scheme is used to minimize an objective function defined by the Bartlett processor output in both beam and mode space. With the input from chirp sonar bathymetry and sediment thickness data, a broadband acoustic field is calculated by a coupled normal mode model and rangedependent geoacoustic properties are inverted. An independent chirp sonar inversion of acoustic impedance profiles was also performed along the same track by using chirp sonar data. The inversion results indicate good agreement between two inversion methods and show their effectiveness if they are used as complementary to each other. The effect of a strongly range-dependent water column on the inversion performance and possible broadband inversion using single hydrophone is also studied. [Work supported by ONR.]

\section{9:30}

2aUW5. Using an adjoint model to invert for unknown perturbations to a presumed environment. Paul Hursky, Michael B. Porter (Sci. Applications Intl. Corp., 1299 Prospect Ave., Rm. 305, La Jolla, CA 92037), Bruce Cornuelle, William S. Hodgkiss, and William A. Kuperman (Scripps Inst. of Oceanogr., Univ. of California, San Diego, La Jolla, CA 92093-0701)

Adjoint modeling is an inversion technique that has the potential to sharply reduce the number of modeling runs typically needed to achieve an inversion. Although adjoint models are being used in oceanographic and meteorological modeling, they seem to have been overlooked by the acoustics community. A single adjoint modeling iteration calculates an adjustment to forward model control inputs (initial conditions and boundary conditions) needed to correct for a mismatch between predicted and measured system parameters. We will present how the adjoint of an acoustic propagation model can be used to trace a mismatch in predicted and measured acoustic parameters back to the perturbations in the environmental parameters needed to correct for the mismatch.
2aUW6. Seabed characterization from statistical analysis of highfrequency data. Samantha J. Dugelay, Richard J. Brothers, and Gary J. Heald (QinetiQ Bincleaves, Newton's Rd., Weymouth, Dorset DT4 8UR, UK)

Results are presented from statistical analysis of experimental and simulated high-frequency seabed backscatter data. The statistical laws employed in the analysis were the Rayleigh distribution, the Rayleigh mixture, and the K-law. The significance of the distribution parameters and their estimation from the data are explained. The link between the parameters and seabed type has been theoretically examined through a detailed study of the variation of statistical laws with grazing angle. Experimental data were collected using a $150-\mathrm{kHz}$ wide-band sonar at three different sites, providing different sediment types. The validity of the above statistical laws was investigated and found to be dependent on sonar geometry. The estimated parameters of the three distributions have been examined for their potential in seabed characterization. Simulations have been compared with experimental data for validation purposes. These results have then been used to investigate the theory developed beyond the scope of the recorded data.

10:00-10:15 Break
10:15

2aUW7. Seabed layer discrimination using normal incidence backscatter returns in the 1-10 $\mathbf{~ H H z}$ frequency band. Gary $\mathrm{J}$. Heald (QinetiQ, Winfrith Technol. Ctr., Winfrith Newburgh, Dorchester, Dorset DT2 8XJ, UK) and Paul C. Hines (Defence Res. Establishment Atlantic, P.O. Box 1012, Dartmouth, NS B2Y 3Z7, Canada)

It has been established that the first and second seabed returns from a monostatic echo sounder can be used as a seabed classification tool. These systems operate on the principle that the first echo is purely monostatic, whereas the second echo can be treated as a bistatic return with a virtual source located at a height of 2-3 times the water depth. Typically these systems operate in the range of $100 \mathrm{kHz}$ so that bottom roughness is the dominant scattering mechanism. In the present work we apply this procedure to lower frequencies. This should provide additional discrimination information since contributions from the seabed volume become significant. Additionally, it provides information about the nature of seabed layers that may be present. However, this comes at the cost of further complicating the model. In this paper we modify the high frequency model to be applicable at lower frequencies and examine the effect of sub-seabed layering.

\section{0:30}

2aUW8. Acoustic scattering from stratified marine sediments. Anatoliy N. Ivakin (APL-UW, 1013 NE 40th St., Seattle, WA 98105, on leave from Andreev Acoust. Inst., Svernika 4, Moscow 117036, Russia, anivakin@yahoo.com)

There are various mechanisms of seabed scattering. Normally, they are attributed to two main types of bottom irregularities: roughness of the sediment interfaces and volume fluctuations of the acoustical parameters. However, there is ambiguity in the description of these irregularities. It is known, e.g., that roughness can be exactly expressed in terms of volume perturbations near the flat interface. On the other hand, there is a class of volume perturbations within continuously stratified media, which can be described in terms of the roughness of isosurfaces of acoustical parameters. Here, all these cases are considered using a unified approach to 
volume and roughness scattering [A. Ivakin, J. Acoust. Soc. Am. 103 (1998)]. This approach unifies also the cases of continuous and discrete stratification (layering). Rough interfaces between layers are replaced by continuous transition layers having rough isosurfaces of the acoustical parameters. First-order perturbations of these parameters are proportional to their vertical gradients and to displacement (roughness) of the isosurfaces within the transition layers. The model gives the volume scattering strength as a function of the wave thickness of the transition layers. In the particular case of low frequencies, where the transition layers are thin in comparison with the wavelength, the model provides an exact transition to existing results for scattering from rough interfaces and, thus, naturally links volume and roughness mechanisms of seabed scattering at low and high frequencies within a unified approach.

\section{1:15}

2aUW11. Validity of backscatter strength models at high (110-170 kHz) frequencies. Sarah J. Page, Richard J. Brothers, and Patricia A. G. Thomson (QinetiQ Bincleaves, Newton's Rd., Weymouth, Dorset DT4 8UR, UK)

Sonar performance models use several low-level acoustic models to predict the effect of the environment on reverberation and noise. Many of these acoustic models, particularly those predicting seabed scattering strength, were developed at lower frequencies than those currently used by both the scientific and mine-hunting communities. This paper examines the popular Applied Physics Laboratory of the University of Washington (APL-UW) acoustic backscatter strength model and tests its validity in the extended frequency band of $110-170 \mathrm{kHz}$. Acoustic and environmental data (gathered as part of a joint program with NATO SACLANTCEN) from several sites are collated and compared in this paper. This provides compelling evidence regarding the validity of the APL-UW backscatter model at these higher frequencies.

\section{0:45}

2aUW9. Extraction of modal backscattering matrix from reverberation data in shallow-water waveguide. Part I. Theory. E. C. Shang (Marine Physical Lab., Scripps Inst. of Oceanogr., La Jolla, CA 92093), T. F. Gao (Inst. of Acoust., Chinese Acad. of Sci., Beijing, PROC), and D. J. Tang (APL, Univ. of Washington, Seattle, WA 98105)

Extracting the bottom backscattering information from reverberation data in shallow-water waveguide is an attractive but difficult issue. In previous works, some a priori assumption (for instance, the Lambert's Law, or the separability of the backscattering matrix) has been made in order to solve this problem. In this paper, new approaches are proposed. The modal backscattering matrix can be extracted directly from reverberation data without any a priori assumption on scattering coefficient. The backscattering information inversion is based on the following three components: (1) mode-filtering at the receiving vertical array, (2) changing the point source depth to obtain different incident mode excitation, and (3) using mode stripping in the waveguide as an additional mode filter. For lower frequency the linear inversion is presented, and for higher frequency the sequential inversion with angle interval smoothing is proposed. [Work supported by ONR.]
11:30

2aUW12. Comparison of bistatic scatter modeling with experimental tank data at $250 \mathrm{kHz}$. Richard J. Brothers, Gary J. Heald (QinetiQ Bincleaves, Newton's Rd., Weymouth, Dorset DT4 8UR, UK), Paul McCloghrie, Philippe Blondel, and Nicholas G. Pace (Univ. of Bath, Bath BA2 7AY, UK)

Experimental data gathered in the laboratory at the University of Bath have been compared to bistatic scattering models. The data were gathered at $250 \mathrm{kHz}$ over different controlled sediment types (sand/gravel/cobble), and at a range of scattering angles using a directional source and omnidirectional receiver. Stringent system calibration was undertaken, along with extensive pre-experimental analysis, in order to guarantee highfidelity scattering data within the confines of a tank environment. Having established the validity of the scattering models, they have been incorporated into a bistatic sonar model. This is achieved by integrating a function that includes the scattering cross-section at each point on the sediment surface, as well as spreading and absorption losses. This model is designed to investigate optimum sonar parameters for the bistatic detection of objects on and in the seabed.

\section{1:00}

2aUW10. Seabed and sub-bottom classification using measurements of normal incidence backscatter from 1-10 kHz. John C. Osler, Paul C. Hines, and Darcy J. MacDougald (Defence Res. Establishment Atlantic, P.O. Box 1012, Dartmouth, NS B2Y 3Z7, Canada)

It has been established that the first and second normal incidence acoustic returned from the seabed can be used as a classification tool to discriminate between different sediments. Typically this is accomplished using monostatic echosounder systems that operate in the range of several $10 \mathrm{~s}$ of $\mathrm{kHz}$ so that bottom roughness at the seabed interface is the dominant scattering mechanism. Model results (Hines and Heald, Proceedings of the Institute of Acoustics Conference on Acoustical Oceanography, Southampton, UK, April 2001) indicate that at lower frequencies, normal incidence returns from the sub-bottom should provide an additional discrimination tool without significantly corrupting the information contained in the interface scatter returns. This paper presents an experimental methodology for making these measurements in the 1 to $10 \mathrm{kHz}$ frequency band using a vertical line array of receivers and a downward-looking superdirective projector array. Results from experiments at the ONR Strataform area and Scotian Shelf will be compared with independent geo-acoustic information.

\section{1:45}

2aUW13. Acoustic field of hydroacoustic antenna arranged near a rough surface. Tran. Huy Dat (Inst. of Hydromechanics of Natl. Acad. of Sci. of Ukraine, Zhelyabov Str. 8/4, Kiev 03035, Ukraine)

The characteristics of an averaged field of a hydroacoustic antenna arranged near a penetrable ocean surface with large-scale random roughness is formulated. The model of an antenna is described as an activity cylindrical surface, restricted absolutely rigid toroid. The given model rather convincingly mirrors actual design features of modern hydroacoustical antennas. The averaged field of a hydroacoustic antenna is analyzed depending on a degree of roughness of boundary, sizes of antennas, field pattern on activity surfaces, way of the view of space and bottom penetration. It showed that, the rough surface could result into differences in distribution of averaged values of a far field of hydroacoustic antennas up to $20 \%$ in comparison with a case of free environments, the value bottom penetration, after which one of the distribution values of the mean characteristics of a near field of the antenna practically coincides appropriate values for a free environment, is established. By the given calculation and discussion, it is followed that, the ocean rough surface could be considered as the natural factor resulting in decreasing of acoustic shadow. Nevertheless, bistatic calculations of coherent field suggest useful strategies for improving in design a new model of the hydroacoustic antenna. 


\title{
Session 2pAAa
}

\section{Architectural Acoustics: Speech Privacy Design in Office Spaces}

\author{
Kenneth P. Roy, Chair \\ Innovation Center, Armstrong World Industries, 2500 Columbia Avenue, Lancaster, Pennsylvania 17603
}

Chair's Introduction-1:00

Invited Papers

1:05

2pAAa1. Speech privacy design and masking sound systems. Kenneth P. Roy (Armstrong World Industries, Innovation Ctr., 2500 Columbia Ave., Lancaster, PA 17403)

The term "speech privacy" when applied to the office building segment must be considered both from the point of view of the person talking and from that of the listener. The achievement of adequate speech privacy for a particular situation is based on both the architectural design of the space, and the acoustical factors including speech and noise characteristic. The signal to noise ratio is the key to privacy performance, and this will be addressed by review of a case study for a recent office application. Electronic masking sound is a key concept in the attainment of speech privacy; and a new approach to masking system design will be reviewed, including an improved speaker concept, source DSP, and modeling tools.

2pAAa2. Speech privacy in closed offices: Comparison of languages and accent. Yong Ma, Daryl J. Caswell (Dept. of Mech. \& Manufacturing Eng., Univ. of Calgary, Calgary, AB T2N 1N4, Canada), Jim T. Goodchild (Smed Intl., Calgary, AB T2C 4T5, Canada), and Liming Dai (Univ. of Regina, SK S4S 0A2, Canada)

Up to now, most objective assessments for speech privacy and speech intelligibility, such as articulation index (AI), speech intelligibility index (SII), early-to-late sound ratio (Clarity), and speech transmission index (STI), are evaluated by subjective measurements primarily based on studies that incorporate only the English language. In today's multicultural environment, it is necessary to study the impact on speech privacy of different languages and accents. In this work, subjective measurements were conducted in closed offices by using English, Mandarin Chinese (a tonal language), and accented English. Both close-set and open-set test materials, including single words, sentences, and articles were employed in the measurement. The results revealed the evaluation differences in speech privacy between two languages, as well as between native English and accented English. The subjective measurement results were also compared with the objective measurement indices AI, STI, and Clarity.

\section{1:45}

2pAAa3. Speech privacy comparison using Articulation Index (AI) and Speech Privacy Noise Isolation Class (NIC) in an open office. Heakyung Yoon and Vivian Loftness (School of Architecture, Carnegie Mellon Univ., MM Hall Rm 410, Pittsburgh, PA 15213, hcy@andrew.cmu.edu)

Two speech privacy measurements, Articulation Index (AI) standardized by the American National Standard Institute in 1969 and Speech Privacy Noise Isolation Class (NIC, Noise Isolation Class Prime) adapted from the Noise Isolation Class (NIC) by U. S. General Services Administration (GSA) in 1979, have been claimed as objective tools to measure speech privacy in open offices. To evaluate which of them is a better tool in terms of speech privacy in a dynamic open office environment, the measurements were taken in the field. AIs and NICs in the current workplace settings as well as in the modifications of partition heights and workplace configurations have been measured following ASTM E1130 (Standard Test Method for Objective Measurement of Speech Privacy in Open Offices Using Articulation Index) and GSA test PBS-C.2, respectively.

\section{2:05}

2pAAa4. Noisy offices for simple tasks and quiet offices for complex tasks? Heakyung Yoon and Vivian Loftness (School of Architecture, Carnegie Mellon Univ., MM Hall Rm 410, Pittsburgh, PA 15213, hcy@andrew.cmu.edu)

Many current office plans are designed as open environments to increase knowledge sharing, which is supposed to increase performance through more informal interactions between workers. However, according to workplace surveys, poor acoustics in open office environments have been reported to decrease workers performances. The literature regarding performance with quiet, ambient, and masking sound in offices show that the kinds of performed tasks in organizations or groups may be the main factor to decide whether open or closed office environments are appropriate. People engaged in simple office tasks perform better with masking sounds 
than with quiet or ambient office sound conditions [Loewen et al., "Cognitive and arousal effects of masking office noise," Environment and Behavior 24, 381-395 (1992)]. However, when people need to use serial-recall functions in their complex tasks, quiet conditions may be better than other sound environments in offices [Banbury et al., "Disruption of office-related tasks by speech and office noise," Br. J. Psychol. 499-517 (1998)].

\title{
Contributed Papers
}

$2: 25$

2pAAa5. Speech levels in open plan offices. Alf Warnock and Wing T. Chu (Inst. for Res. in Construction, Natl. Res. Council, Canada)

A key variable for predicting speech privacy in open offices is the level of speech used. Since the environment influences vocal effort, speech levels needed to be measured in typical open office situations to more accurately calculate speech privacy in such offices. This paper describes measurements of voice levels used in face-to-face conversation in nine open offices in the Ottawa/Hull Area. Speech from 118 subjects was recorded and analyzed. Average voice levels in each office ranged from 48 to $55 \mathrm{dBA}$. The overall mean was $51 \mathrm{dBA}$ with a standard deviation of 3.4 dBA. Levels from the current study were significantly lower than those in ASTM standard E1130, but the overall average and spectrum agree well with Pearsons' results for casual conversation (K. S. Pearsons, R. L. Bennett, and S. Fidel, "Speech levels in various noise environments," EPA6001-77-025, U. S. Environmental Protection Agency, May 1977). The paper describes the procedures and the results obtained. This work was done as part of a project for Public Works and Government Services Canada.

\section{2:40}

2pAAa6. Directivity of human talkers. Wing T. Chu, Alf Warnock (Inst. for Res. in Construction, Natl. Res. Council, Canada), and JeanCharles Guy (INSA de Lyon, France)

As well as the level of speech, the directivity of the sound field surrounding the human head is important when estimating speech privacy in open offices. Information on this subject was found to be sparse so the sound field surrounding human talkers was recorded by two sets of eight microphones fixed on two orthogonal meridian arcs in an anechoic chamber. Talkers were positioned at the center of the two arcs of the microphone arrays and rotated through six positions at $15^{\circ}$ increments to survey the whole sound field. Twenty male and twenty female subjects were used. No significant differences in directivity were detected between male and female talkers although the mean spectra were different. There was good agreement between the field and laboratory measurements of the male voice spectra though some differences were found for the female voice spectra. No significant differences in directivity or frequency content were detected between English and French talkers. Similar directivities were obtained for the average human talker and the Bruel and Kjaer Head and Torso Simulator. Agreement with other published information is fairly good. This work was done as part of a project for Public Works and Government Services Canada.

\section{Session 2pAAb}

\section{Architectural Acoustics: The Technical Committee on Architectural Acoustics Vern O. Knudsen Distinguished Lecture}

\author{
David Lubman, Chair \\ 14301 Middletown Lane, Westminster, California 92683
}

Chair's Introduction-3:30

Invited Paper

3:35

2pAAb1. Acoustic concerns related to multi cultural societies. Anders Chr. Gade (Oersted, DTU, Section of Acoust. Technol., Tech. Univ. of Denmark, Bldg. 352, DK-2800 Lyngby, Denmark, acg@oersted.dtu.dk)

Immigration has increased cultural diversity in western societies. The process of integrating immigrants into their host countries can be smoothed if acousticians learn to recognize (1) the acoustic traditions of immigrant cultures and (2) the specific acoustic needs of the new society members. Two related projects are discussed. The "Cahrisma" project (Conservation of Acoustical Heritage by the Revival and Identification of the Sinan's Mosque Acoustics) is sponsored by the European Commission and carried out in cooperation among researchers in Turkey, Malta, Italy, France, Switzerland, and Denmark. Its purpose is to combine visual and acoustical concerns in the identification, conservation, and restoration of architectural heritage. It focuses on the famous Turkish mosques of the Osmannic architect Sinan. Some of the acoustic features of these large domed buildings and of muslim liturgy will be presented. Another project was conducted by students at the Danish Technical University, who found that speech intelligibility for non-native listeners is much more sensitive to poor speech conditions than for native listeners. Also discussed are the unique features of culturally based urban soundscapes. It is suggested that these soundscapes can provide comfort to recent immigrants by increasing their sense of being "at home." 


\title{
Session 2pAB
}

\section{Animal Bioacoustics: Topics in Animal Bioacoustics}

\author{
David A. Mann, Chair \\ College of Marine Science, University of South Florida, 140 Seventh Avenue, South, Saint Petersburg, Florida 33701
}

Contributed Papers

$1: 30$

2pAB1. A comparison of visual and acoustic marine mammal monitoring methods. Adam S. Frankel and Kathleen Vigness-Raposa (Marine Acoust., Inc., 901 N. Stuart St., Ste. 708, Arlington, VA 22203)

Concern about the potential effect of increased oceanic noise on marine mammals has led to the consideration of a variety of at-sea marine mammal monitoring methods. A recent marine mammal survey utilized both visual observation and passive acoustic monitoring. Visual observations were conducted using $7 \times 50$ binoculars during the day and generation III night vision devices (NVDs) at night. Acoustic data were collected with a towed hydrophone array and analyzed with a customized, PC-based acoustic workstation. Seventy-seven visual sightings were made. Forty sightings were made at night and 37 were made in daylight. The effective range of the NVDs was estimated through observations of a target at known distances; $50 \%$ detection rating was achieved at $130 \mathrm{~m}$. There were 98 acoustic detections. Of the 77 visual sightings, 42 were also detected acoustically. Six sightings were made without comparable acoustic detection, and 19 sightings were made when there was no acoustic monitoring. Conversely, 56 acoustic detections were made without visual sightings. These data suggest that, for species that commonly vocalize, a combined acoustic/visual survey will increase detection probabilities. Furthermore, night vision devices have the potential to be an effective observation tool for marine mammals. [Work sponsored by ONR.]

\section{$1: 45$}

2pAB2. Sonic boom penetration under a wavy air-water interface. H. K. Cheng (Univ. of Southern California, Los Angeles, CA 90089), Adam Fincham (Laboratoire des Ecoulements Géophysiques et Industriels, CNRS-UJF-INPG, BP 53, Grenoble 38041, France), Tony Maxworthy (Univ. of Southern California, Los Angeles, CA 90089), and John R. Edwards (Environ. Management Div., Space and Missle Systems Ctr., Los Angeles AFB, CA 90245)

The interaction of a sonic boom with a wavy air-water interface is investigated theoretically and experimentally to ascertain its effect on sonic boom noise penetration in deep water. The Mach number is assumed to be less than the water-to-air sound speed ratio of 4.53 . Unlike the problem with a flat (nonwavy) interface, the interaction with a surfacewave train can produce everfescent (nonattenuating) wave components, in addition to evanescent wave components underwater. The overall effect results in an attenuation rate with a depth which is much lower than predicted by the flat-ocean (Sawyers) model; it overwhelms the flat-ocean wavefield in deep water, even though it has a secondary effect at the surface. The expectation is substantiated by both analysis and laboratory measurements. The latter were performed in a water channel with wavemakers and projectile over flights. Detailed measurements confirm both the wave packet characteristics of the deep water waveform and the frequency downshift property predicted by the theory. Underwater soundpressure level, frequency range, and waveform characteristics from sonic booms of aircraft and space-launch operations are examined with the wavy-ocean model.

\section{2:00}

2pAB3. Using high-frequency acoustics to protect marine mammals. Joseph S. Johnson (Chief of Naval Operations, N7747, 2000 Navy Pentagon, Washington, DC 20350-2000), William T. Ellison (Marine Acoust., Inc., Litchfield, CT 06759), Peter Stein (Sci. Solutions, Inc., Nashua, NH 03063), and Clayton H. Spikes (Marine Acoust., Inc., Arlington, VA 22203)

A joint industry-government research effort has designed, developed, and tested a high-frequency marine mammal monitoring (HF/M3) system to ensure that marine mammals are not exposed to high levels of lowfrequency sonar signals. The system's nominal operating characteristics are $30-40 \mathrm{kHz}$ frequency, a variable bandwidth of $1.5-6 \mathrm{kHz}, 3 \%-4 \%$ duty cycle, $10-40 \mathrm{~m}$ pulse length, 3-4 s pulse repetition rate, and $220 \mathrm{~dB}$ (re: $1 \mu$ Pascal at $1 \mathrm{~m}$ ) source level. HF/M3 operating parameters are based on in situ acoustic modeling and low-power probe-pulse signals, which define the local ocean acoustic environment. Operating protocols include source level adjustments to ensure approaching marine mammals or sea turtles are unaffected by HF/M3 signals. System capability has been tested and evaluated in 7 sea trials. Estimated probabilities of detection for a $20-\mathrm{m}$ stationary whale at $160 \mathrm{~m}$ depth are from $98 \%$ at $1-\mathrm{km}$ range to $72 \%$ at $2-\mathrm{km}$ range. The ability to track animals via multiple detections virtually eliminates randomly distributed false alarms. Single-ping probabilities of detection suggest that any marine mammal (and possibly sea turtle) within $1 \mathrm{~km}$ can be expected to be detected multiple timesapproximately once every $50 \mathrm{~s}$.

\section{2:15}

2pAB4. Interactions of Florida manatees (Trichechus manatus latirostris) with simulated fishing gear and a pinger: Implications for preventing entanglements. A. E. Bowles, C. Alves, and R. A. Anderson (Hubbs-Sea World Res. Inst., 2595 Ingraham St., San Diego, CA 92109)

Nine stranded, rehabilitated and captive-born Florida manatees (Trichechus manatus latirostris) were exposed to simulated crab-pots and gillnetting to quantify interactions (70 trials). A pinger (Dukane-Seacom Netcom 1000, $300 \mathrm{~ms}$ ping at $4 \mathrm{~s}$ intervals, level $\sim 130 \mathrm{~dB}$ re $1 \mu \mathrm{Pa}$ from 10 to $80 \mathrm{kHz}$ ) was presented with and without the crab pot during 7 of the trials. Manatees were observed for $30 \mathrm{~min}$ (baseline), then exposed to the object for $30 \mathrm{~min}$. During exposures, they manipulated objects extensively, with little or no evidence of neophobic avoidance. Most contacts (70\%) were made with the mouth; the remainder involved the pectoral flippers, body, and paddle. Entanglements sufficient to require human intervention occurred in four trials. Manatees spent $36 \%$ more time in the test area when objects were present than during baseline trials, indicating a positive motivation to manipulate. The pinger stimulated brief startle reactions in two of four female manatees but in none of five males. Crab pots with pingers were contacted as much as the simulated crab pot alone $(51 \%$ vs $41 \%$ by females). This motivation to manipulate suggests that manatees cannot be protected from fishing gear by pingers. 


\section{2:30-2:45 Break}

\section{2:45}

2pAB5. Hyperbolic location errors due to insufficient numbers of receivers. John L. Spiesberger (Dept. of Earth and Environ. Sci., Univ. of Pennsylvania, 240 S. 33rd St., Philadelphia, PA 19104-6316)

Animal locations are sometimes estimated by measuring the difference in travel times of their sounds at pairs of receivers. Ideally, each difference specifies the animal's location to a hyperboloid, and sufficient numbers of intersecting hyperboloids specify the location. Most bioacoustic publications state that one needs three receivers to locate animals in two spatial dimensions and four receivers in three spatial dimensions. However the actual numbers of receivers required for locations in two and three dimensions are four and five, respectively. Significant location errors can result from using insufficient numbers of receivers. Methods are provided for determining locations of ambiguous source-location regions.

\section{3:00}

2pAB6. Songlike vocalizations and infrasound from the Sumatran rhinoceros. Elizabeth von Muggenthaler (Fauna Commun. Res. Inst., P.O. Box 1126, Hillsborough, NC 27278), Paul Reinhart (Cincinnati Zoo, 3400 Vine St., Cincinnati, OH 45220), and Brad Lympany (Asheboro, $\mathrm{NC})$

Within the last ten years the Sumatran rhino (Dicermoceros sumatrensis) population has dropped 50\%, and only 200-300 individuals are left in the world. The oldest living species of rhino in evolutionary terms, Sumatran rhinos are solitary, although males and females are seen together during courtship. Their native habitat is dense tropical forest and mountain moss forest. They are the smallest living rhino, standing $0.09-1.5 \mathrm{~m}$ tall, and are covered in course, reddish-brown hair. Three Sumatran rhinos, housed at the Cincinnati Zoo, were recorded from 1-3 $\mathrm{m}$. Two Statham Radio microphones, and two Sony TCD-D8 DAT recorders recorded from
$9 \mathrm{~Hz}$ to $22 \mathrm{KHz}$. Analysis, including FFTs, spectrographs, and filtering, were performed using National Instrument's Polynesia. The rhinos proved to be extremely vocal, producing signals almost constantly. Distinct calls, including several types of "eeps," $70 \mathrm{~Hz}-4 \mathrm{kHz}$ (57-92 dB); "whales," $100 \mathrm{~Hz}-3.2 \mathrm{kHz}(87 \mathrm{~dB})$; and "whistle-blows," $17 \mathrm{~Hz}-8 \mathrm{kHz}(100 \mathrm{~dB})$ were discovered. The "whistle-blow" has high $\mathrm{dB}$ infrasound that would be advantageous for use in the rhino's forest habitat. Some Sumatran rhino vocalizations sound similar to and resemble (under analysis) some humpback whale signals.

\section{$3: 15$}

2pAB7. The felid purr: A healing mechanism? Elizabeth von Muggenthaler (Fauna Commun. Res. Inst., P.O. Box 1126, Hillsborough, NC27278, L@animalvoice.com)

A current hypothesis suggests the purr indicates contentment, however, cats purr when they are severely injured or frightened. Forty-four felids were recorded including cheetahs, ocelots, pumas, domestic cats, and servals. A Sony TCD-D8 Digital Audio Recorder (DAT) and Statham Radio microphones recorded the purrs. FFTs and spectrographs were performed using National Instrument's Polynesia. An accelerometer was also used to measure domestic cat purrs. Every felid in the study generated strong frequencies between 25 and $150 \mathrm{~Hz}$. Purr frequencies correspond to vibrational/electrical frequencies used in treatment for bone growth/ fractures, pain, edema, muscle growth/strain, joint flexibility, dyspnea, and wounds. Domestic cats, servals, ocelots, and pumas produce fundamental, dominant, or strong frequencies at exactly $25 \mathrm{~Hz}$ and $50 \mathrm{~Hz}$, the two low frequencies that best promote bone growth/fracture healing [Chen et al., Zhong. Wai Ke Za Zhi. 32, 217-219 (1994)]. These four species have a strong harmonic exactly at, or within $2 \mathrm{~Hz}$ of $100 \mathrm{~Hz}$, a frequency used therapeutically for pain, edema, wounds, and dyspnea. An internal healing mechanism would be advantageous, increasing recovery time and keeping muscles and bone strong when sedentary. [Published with permission from the New Zealand Veterinary Journal; work supported by Endevco.]

Session 2pAO

\title{
Acoustical Oceanography and Physical Acoustics: Turbulence and Finestructure Studies I: Surface and Bottom Boundary Layer Turbulence
}

\author{
James F. Lynch, Chair \\ Woods Hole Oceanographic Institution, 203 Bigelow Building, Woods Hole, Massachusetts 02543
}

Invited Papers

1:00

2pAO1. Dynamics of bottom boundary layers in the coastal ocean. John Trowbridge (Woods Hole Oceanogr. Inst., Woods Hole, MA 02543, jtrowbridge@whoi.edu)

The bottom boundary layer is the region adjacent to the sea floor, with a thickness typically on the order of meters to tens of meters, where turbulence generated by bottom drag produces vertical mixing of mass, heat, and momentum. Oceanic boundary layers are influenced not only by turbulent mixing, but also by planetary rotation, stratification, topography, surface waves, internal waves, and interaction with the erodible sea floor. Historically, understanding of the oceanic bottom boundary layer has been based on classical results in engineering and meteorology. However, recent theory and measurements have revealed unique features of oceanic boundary layers. Outstanding problems include measuring the interaction of the flow with the erodible sea floor; measuring the spatial scales of turbulent motions that accomplish vertical transport of mass, heat, and momentum; and understanding the processes that control the intensity and scale of boundary layer turbulence, particularly in stable stratification. 
2pAO2. Laboratory and field measurements of breaking-induced turbulence: Implications for ocean acoustics. W. Kendall Melville (Scripps Inst. of Oceanogr., UCSD, La Jolla, CA 92093-0213)

The entrainment of air by breaking surface waves, its breakup into bubbles, and their transport in the surface layers by breakinginduced turbulence and Langmuir circulations have important consequences for active and passive acoustical oceanography. Recent laboratory and field work related to these issues will be presented. The description of breaking-induced turbulence and air entrainment by just field experiments is very difficult and there are some advantages to separating the issues into those that must be addressed in the field and those that are better studied in the laboratory. For example, the statistics of breaking cannot be resolved by laboratory measurements, whereas the detailed structure of the turbulence is probably better studied in the laboratory. New results from airborne measurements of breaking statistics, and laboratory (and field) measurements of breaking-induced turbulence will be presented and their implications for upper ocean acoustics will be discussed. Similarities between breaking-induced turbulence and Langmuir circulations will be discussed. [Work supported by ONR and NSF.]

2pA03. 2D imaging of breaking waves with a pulse-coherent phased array. Jerome Smith (Scripps Inst. of Oceanogr., 0213 UCSD, La Jolla, CA 92093-0213)

Breaking waves represent a serious challenge in the study of the air-sea interface. Entrainment of air and the expulsion of droplets and particles by breaking waves are of primary importance to material fluxes in particular and air/sea exchanges in general. Observations of motion near the surface is key to understanding the kinematics and dynamics of this elusive interface. Unfortunately, it is nearly impossible to obtain measurements closer than a meter or two below actively breaking wave crests in the open ocean. Here, measurements from an up-looking phased-array sonar system are presented. The system provides measurements over pies about 22 degrees wide by $16 \mathrm{~m}$ maximum range, with resolution 1.5 degrees by $5 \mathrm{~cm}$. The instrument was $13.5 \mathrm{~m}$ below the mean surface, so the measurement cell size near the surface is about $5 \mathrm{~cm}$ (vertical) by $30 \mathrm{~cm}$ (along-wind). Sequences of intensity images can be analyzed for motion and relative scatterer (bubble) density. Data presented were obtained as a wave of about $2.5 \mathrm{~m}$ height crest to trough passed through the field of view while breaking (one of several breakers captured over a two-week deployment from R/P FLIP).

2pA04. Doppler measurement of velocity structure beneath surface gravity waves. David Farmer, Johannes Gemmrich, and Svein Vagle (Inst. of Ocean Sci., Sidney, BC V8L 4B2, Canada)

The acoustic Doppler method has proved particularly useful for the investigation of small scale velocity structure associated with wave motions, shear instability, and turbulence. The incoherent bistatic approach permits measurement of the velocity field at meter scales within the crests of large and steep waves, allowing direct comparison with predictions in the region where theory is most likely to break down. At the other end of the scale, coherent Doppler measurement at megahertz frequencies can be used to identify and estimate the magnitude of turbulence beneath breaking waves. We contrast these different approaches using observations with a bistatic incoherent Doppler sonar placed on the floor of the North Sea and an array of monostatic coherent sonars deployed on the ocean surface off the California coast. The observations illustrate the power of Doppler techniques in resolving various aspects of wave dynamics which have been inaccessible using traditional methods.

2pA05. Characteristics of turbulence in the surf zone. John Trowbridge (Woods Hole Oceanogr. Inst., Woods Hole, MA 02543, jtrowbridge@whoi.edu) and Steve Elgar (Woods Hole Oceanogr. Inst., Woods Hole, MA 02543)

Near-bottom measurements from an array of acoustic Doppler velocimeters over a sand beach produce direct estimates of turbulent shear stress, by means of a differencing procedure that rejects contributions from surface waves, and indirect estimates of dissipation, by means of an inertial-range model. Measurements of winds, waves, and currents provide a context for analysis of the turbulence measurements. The analysis focuses on an alongshore momentum equation, semiempirical relationships between turbulent shear stress and alongshore velocity, and simplified budgets for energetics. The records are dominated by three events when the instruments were in the surf zone. Near-bottom turbulent shear stress was well correlated with, but smaller than, the sum of wind and wave forcing. Vertical gradient of alongshore velocity was larger than indicated by the Prandtl-Karman law of the wall. Drag coefficient was smaller during breaking waves than during unbroken waves. Shear production of turbulent kinetic energy was approximately equal to dissipation, and both were two orders of magnitude smaller than the depth-averaged rate at which the shoaling wave field lost energy to breaking, indicating that breaking-induced turbulence did not penetrate to the seabed. 


\title{
Contributed Papers
}

3:55

2pA06. Coherent Doppler profiler measurements of near-bed suspended sediment fluxes. Carolyn E. Smyth and Alex E. Hay (Dalhousie Univ., 1355 Oxford St., Halifax, NS B3H 4J1, Canada, csmyth@phys.ocean.dal.ca)

Vertical profiles of vertical turbulence intensity and vertical sediment fluxes were collected by an acoustic Coherent Doppler Profiler during two experiments: at Queensland Beach, NS and SandyDuck97. Measurements are investigated as a function of bedstate from low energy vortex ripples to high energy flat bed. Ensemble-average vertical turbulence intensity profiles exhibit a peak within the wave boundary layer at heights of $O(1 \mathrm{~cm})$ above bottom for all bedstates. Peak average turbulence intensities are relatively independent of bedstate, varying by no more than $50 \%$ despite a factor of 7-10 variation in average wave energy. This otherwise remarkable observation can be understood from the corresponding decrease in the physical roughness of the bed, associated with the different observed bedstates. Estimated wave friction factors are highest for low energy rippled beds and smallest for flat bed conditions. Estimates of the vertical suspended sediment flux partitioned into mean, wave, and turbulent components, show that in general, there is a balance between upward fluxes and downward settling; except immediately above the bed, and except for the case of a stationary ripple field. The suspended sediment flux coherence indicates enhancement at incident wave frequencies, with the largest coherence for flat bed conditions very near the bed.

4:10

2pA07. Measurements of turbulence in the wave boundary layer using high frequency acoustics. Eric Terrill, Ronan Gray, and Ken Melville (Scripps Insitution of Oceanogr., Marine Physical Lab., Mail Code 0213, La Jolla, CA 92093-0213)

The properties of the turbulent boundary layer at the air-sea interface exhibits departures from classical wall bounded shear flows as a result of the free surface characterized by surface waves. As surface waves grow in the presence of wind, they become unstable and break, which leads to the injection of turbulence and bubbles into the water column. While an understanding of these physical processes is critical to fields of oceanography and mid-/high-frequency ocean acoustics, there remains much to be learned due in part to the difficulty of obtaining turbulence measurements at the air-sea interface as well as signal processing issues associated with separating turbulent water motions from wave orbital motions. One maturing solution to this measurement problem is the use of $O(1) \mathrm{MHz}$ pulse to pulse coherent Doppler sonars that allow direct wave number measurements in the inertial subrange of the turbulence. Recent field measurements of near-surface turbulence have been conducted by our group with this sonar technology both offshore Pt. Conception California and Hawaii. Measurement results will be discussed, including observations regarding the relationship between the intermittancy of the turbulent dissipation to the overlying surface wave field. [Work supported by ONR and NSF.]

$4: 25$

2pA08. Observations of turbulence in ocean wave boundary layers. Timothy P. Stanton (Oceanogr. Dept., NPGS, Monterey, CA 93943)

A bistatic geometry, coherently sampled, acoustic Doppler profiler has been developed to measure turbulent stresses in wave-forced ocean boundary layers. The profiler is typically configured to measure over-resolved profiles of three-component velocity vectors sampled in $O\left(2 \mathrm{~cm}^{3}\right)$ sample volumes at $20 \mathrm{~Hz}$ over a $60 \mathrm{~cm}$ range above the ocean bed. Examples of bottom boundary layer observations made at Duck, NC and Monterey, CA during two field experiments illustrate approaches to measuring Reynolds stresses and shear in thin oscillatory boundary layers over a rippled bed. During the ONR-funded Shoaling Waves Experiment (SHOWEX) the profiler was mounted on an instrumented frame at $12 \mathrm{~m}$ depth and continuously measured velocity profiles and changes in waveinduced bed morphology through a succession of storms over a two month period. In the second example, observations of mean currents, waveinduced velocities, and turbulence were made in the surf zone just offshore from a steep-sloping beach.

Panel Discussion

\section{Session 2pBB}

\section{Biomedical Ultrasound/Bioresponse to Vibration: Topical Meeting on Physics of Ultrasound in Relation to the Biology of its Therapeutic Effects: Ultrasound with Adjunctive Agents}

\author{
Lawrence A. Crum, Chair \\ Center for Industrial and Medical Ultrasound, Applied Physics Laboratory, University of Washington, 1013 NE 40th Street, \\ Seattle, Washington 98105
}

Chair's Introduction-2:00

Invited Papers

2:05

2pBB1. A possible physical mechanism of ultrasound-activated gene delivery: Shear stress generated by microstreaming. Junru Wu (Dept. of Phys., Univ. of Vermont, Burlington, VT 05405, jwu@zoo.uvm.edu)

Gene therapy provides potentially effective treatment of many diseases. Sonoporation and ultrasound induced transfection can promote the uptake and expression of foreign DNA by a cell. In vitro studies [Bao et al., Ultrasound Med. Biol. 23, 953-959 (1997); Greenleaf et al., Ultrasound Med. Biol. 24, 587-595 (1998); Ward et al., J. Acoust. Soc. Am. 105, 2951-2957 (1999); Ultrasound Med. Biol. 26, 1169-1175 (2000); Guzman et al., J. Acoust. Soc. Am. 110, 588-596 (2001)] have shown that the presence of contrast 
agents dramatically enhances the efficiency of the transfection and also reduces the required acoustic pressure. Recent experimental studies and numerical calculations also demonstrated that the shear stress associated with microstreaming surrounding encapsulated bubbles of contrast agents near living cells driven by $0.1 \mathrm{MPa}$ acoustic pressure amplitude ultrasound a $1 \mathrm{MHz}$ or $2 \mathrm{MHz}$ may be large enough to generate reparable sonoporation to the cells. This presentation will review above-mentioned publications and discuss the possible physical mechanism involved.

2pBB2. Ultrasound contrast agents to enhance and monitor therapy. Katherine Ferrara, James Chomas, Donovan May, John Allen (Dept. of Biomed. Eng., Univ. of California Davis, Davis, CA 95616), Rachel Pollard, and Erik Wisner (Univ. of California Davis, Davis, CA 95616)

New techniques for the assessment of tissue perfusion and the local delivery of drugs have been developed using ultrasound contrast agents. A perfusion estimator, based on the destruction and wash-in of ultrasound contrast agents, has been developed and applied to tumor models. This technique requires the transmission of a train of pulses with varying amplitude, frequency, and phase within the train. High-amplitude and low-frequency pulses are applied to destroy the agent, and low-amplitude higher-frequency pulses are used to monitor the refresh of contrast agents into the sample volume. The signal processing techniques applied following data acquisition utilize the subharmonic component of the received signal. Mechanistic studies of contrast agent destruction, a Rayleigh-Plesset based signal model, and experimental data acquired from a rat tumor model will be included in our presentation. In addition, studies of the local delivery of drugs using oil-based drug delivery vehicles have been conducted. Results of these studies demonstrate that the probability of agent fragmentation and the size of the resulting fragment can be controlled using ultrasonic parameters. [We acknowledge the support of NIH CA 76062.]

2pBB3. Equilibrium loading of cells with molecules by ultrasound: Effects of molecular size, acoustic energy, and cell-to-bubble ratio. Mark Prausnitz, Hector Guzman, and Robyn Schlicher (School of Chemical Eng., Georgia Tech, Atlanta, GA 30332-0100)

Ultrasound has been shown to disrupt cell membranes and thereby load cells with molecules. In this study, we quantified levels of cellular uptake of calcein $(0.6 \mathrm{kDa})$, bovine serum albumin $(66 \mathrm{kDa})$, and two dextrans (42 and $464 \mathrm{kDa})$ into DU145 prostate cancer cells using $500 \mathrm{kHz}$ ultrasound in the presence of Optison contrast agent. The results demonstrated that levels of uptake (i) were similar for all four molecules at the same ultrasound conditions and (ii) for some cells reached thermodynamic equilibrium with the extracellular solution. Over a broad range of acoustic conditions, these levels of uptake, as well as loss of cell viability, were shown to correlate with acoustic energy exposure. In a separate set of experiments, increasing cell concentration was shown to increase both viability and uptake, and increasing Optison bubble concentration decreased viability with no significant effect on uptake. As a parameter that can unify these two sets of data, the cell-to-bubble ratio was found to correlate well with both cell viability and the number of cells with uptake normalized relative to the number of Optison bubbles provided, which can be viewed as a measure of bubble "efficiency."

\section{3:05-3:20 Break}

2pBB4. Three variations on a theme: Ultrasound permeabilization of biologically interesting membranes. Pierre D. Mourad (Ctr. for Medical Acoust., Appl. Phys. Lab. and Dept. of Neurological Surgery, Univ. of Washington, Seattle, WA 98195)

This presentation focuses on three applications of therapeutic ultrasound for the purpose of permeabilizing membranes, with an emphasis, where possible, on the biology as well as the physics of the processes involved. In the first instance, ultrasound successfully and transiently opens the blood-brain barrier to the flux of blood-borne chemical dyes in vivo, as documented grossly, and with light and electron microscopy. In the second example, ultrasound transiently disrupts the membrane coating of a novel biopolymer tablet in vitro in order to release insulin carried within the tablet. The membrane consists of a series of self-assembling monolayers. In the final example, ultrasound acts synergistically with a novel polymer to permeabilize model lipid bilayers. The novelty here is that the effect is $\mathrm{pH}$-dependent, due to the $\mathrm{pH}$-dependence of the conformation of the polymer, and, that the polymer can be both targeted to cell surfaces with appropriate receptors, and can carry drugs or DNA. [Work supported by NIH and DARPA. I would like to thank my colleagues at CIMU and the Department of Neurological Surgery for their support in this work.]

\section{3:40}

2pBB5. Therapeutic applications of microbubbles. Evan C. Unger, Terry O. Matsunaga, Thomas P. McCreery, Robert Sweitzer, Patricia Schumann, Rachel Quigley, and Reena Zutshi (ImaRx Therapeutics, Inc., 1635 E. 18th St., Tucson, AZ 85719)

While microbubbles have been developed for diagnosis as ultrasound contrast agents, the greatest potential of these agents may lie in therapy. Microbubbles have extensive applications for treating vascular thrombosis, drug and gene delivery. Targeted microbubbles containing ligands for cell or site-specific recognition have been developed. These agents function like "smart bubbles" to home in on their targets. One such agent, MRX-408, a microbubble bearing targeting ligands directed to the activated receptor of platelets has undergone extensive pre-clinical testing. Recent studies comparing Sonothrombolysis with MRX-408 and nontargeted microbubbles show significantly more clot lysis with targeted as compared to nontargeted microbubbles. The targeted bubbles effectively concentrate cavitation nuclei within the clot. Additional targeted microbubbles for drug and gene delivery are also under development. 
2pBB6. Sonodynamic treatment with cavitation-promoting agent activated by second-harmonic superimposed progressive waves. Shin-ichiro Umemura, Ken-ichi Kawabata, Nami Sugita, and Kazuaki Sasaki (Central Res. Lab., Hitachi, Ltd., Kokubunji, Tokyo 185-8601, Japan, sumemura@crl.hitachi.co.jp)

Sonodynamic therapy has been proposed based on the finding that certain chemicals such as hematoporphyrin are activated by acoustic cavitation and thereby induce a significant antitumor effect. It has also been found that sonodynamically active cavitation can be induced at a relatively low ultrasonic intensity through superimposing the second harmonic onto the fundamental. An in vivo as well as an in vitro cavitation threshold was an order of magnitude lowered by second-harmonic superimposition especially when combined with a cavitation-promoting sonochemically active agent such as rose bengal. Amphiphilic derivatives of rose bengal were synthesized so as to obtain tumor accumulating agents of this kind for sonodynamic tumor treatment. The tissue distribution of the derivatives in mice was investigated and those having a normal alkylic chain with 10-15 carbon atoms were proved to accumulate well in tumor tissues. With an administration of one of the derivatives, experimental murine tumors were treated with secondharmonic superimposed progressive waves at 0.5 and $1 \mathrm{MHz}$. The tumor growth was significantly suppressed by sonodynamic treatment in this combination while ultrasound alone showed only a slight effect. [Work supported in part by the National Research and Development Program for Medical and Welfare Apparatus, Japan.]

4:20-4:25

Break

4:25-5:25

Panel Discussion

$5: 25-5: 30$

Closing Remarks

TUESDAY AFTERNOON, 4 DECEMBER 2001

ROOM 220, 1:30 TO 3:45 P.M.

\title{
Session 2pEA
}

\section{Engineering Acoustics: Sensors and Sources in Air}

\author{
Ahmet Selamet, Cochair \\ Center for Automotive Research, The Ohio State University, 930 Kinnear Road, Columbus, Ohio 43212 \\ Louis N. Cattafesta III, Cochair \\ Department of Aerospace Engineering, Mechanical and Engineering Sciences, University of Florida, Box 116250, \\ Gainesville, Florida 32611
}

\section{Contributed Papers}

$1: 30$

2pEA1. System for measurement of microphone distortion and linearity at very high sound levels. Erling Frederiksen (Brüel \& Kjaer, 2850 Naerum, Denmark)

Even if sound measurements are made over a very wide dynamic range, say from $0 \mathrm{~dB}$ to $180 \mathrm{~dB}$, the applied instruments are typically tested only at one level, which is most often within the range between 94 $\mathrm{dB}$ and $124 \mathrm{~dB}$. Is this enough? Some types of microphone have probably never been tested at medium and high levels, as neither standardized nor generally agreed methods are described or available. However, today aerospace organizations, accreditation bodies and laboratories performing pattern approval of instruments ask for documentation, methods, and test equipment. Therefore, B\&K decided to develop a system for generation of sound pressure over a wide dynamic range for routine testing of measurement microphones and pressure transducers. This paper describes the design and the properties of the system, which generates high pressures by a system of coupled tubes with standing waves. The general operation principle is not new, but the system, which can operate upon an ordinary laboratory table, is refined and optimized for a generation of levels as high as $174 \mathrm{~dB}$ at $500 \mathrm{~Hz}$ with a very low distortion (attention is paid to third harmonic distortion, which indicates lack of linearity).

\section{1:45}

2pEA2. Measurement of background noise in piezoelectric and electret condenser microphones. Allan J. Zuckerwar (NASA Langley Res. Ctr., M.S. 493, Hampton, VA 23681) and M. Roman Serbyn (Morgan State Univ., Baltimore, MD 21251)

An upgraded version of a previously described acoustic isolation vessel [K. C. T. Ngo and A. J. Zuckerwar, J. Acoust. Soc. Am. 93, 2974-2980 (1993)] has been used to investigate the background noise in piezoresistive and electret condenser microphones over the frequency range $1 \mathrm{~Hz}-100$ $\mathrm{kHz}$. The test microphones represent four different manufacturers. Theoretical models for predicting the noise characteristics of piezoresistive (nonreciprocal) and electret condenser (reciprocal) microphones are proposed. In particular, the results of 1/f noise measurements on these microphones are compared with those previously obtained from air condenser microphones. 
tum equation, like $f_{i}$, and is equivalent to an applied force/unit volume resulting in sound-generating fluid acceleration, while (c) appears in it only in derivative form. Hence (b) is an attractive alternative to the customary (c) because of that basic relationship which has simple physical appeal.

\section{2:45-3:00 Break}

The need for noise source localization and characterization has driven the development of advanced sound field measurement techniques using microphone arrays; however, the cost and complexity of these systems limit their widespread use. One common application is the study of aircraft noise in wind tunnels. The arrays typically consist of moderate numbers $(<40)$ of conventional condenser microphones and range in size from several inches to several feet. Arrays consisting of more microphones and/or the use of multiple arrays may be needed to analyze complex multipole noise sources. However, the feasibility of scaling the current technology to multiple arrays with large numbers of microphones is limited by the cost per channel, array mobility, and data handling efficiency. A reduced cost, mobile, high speed 16-channel array has been constructed to demonstrate key technologies to address these scaling issues. The system is based on a hybrid package that combines a silicon piezoresistive microphone and amplification circuitry. Each package is socketed into a printed circuit board, providing electrical connections and structural support. High speed data acquisition and signal processing hardware is used to generate the array response in near real time. The results of the initial array calibrations are presented.

\section{2:15}

2pEA4. Effects of temperature and humidity on the bending stiffness of a sheet of paper measured by laser ultrasonics. Matthew A. Cornwell and Yves H. Berthelot (Woodruff School of Mech. Eng., Georgia Inst. of Technol., Atlanta, GA 30332-0405)

Laser ultrasonics has great potential for monitoring the bending stiffness of paper during on-line operations. The temperature and humidity conditions at the paper have a significant effect on the bending stiffness, and there is a need to quantify this dependence before an on-line system can be deployed. A laser pulsed source illuminates a point on a paper sample placed inside a controlled environmental chamber and generates ultrasound that is detected by a fiberoptic interferometer. The waveform is analyzed to extract the frequency dependence of the group velocity of the A0 lamb mode, and the corresponding bending stiffness. Three different methods of signal analysis are compared: the complex wavelet transform, the short-time Fourier transform, and the phase-unwrapping method, which requires measurements at two locations. The error bars associated with the bending stiffness are estimated both for the intrinsic experimental errors and for the point to point variablity in the paper itself. Results are presented for temperature ranging from $25^{\circ} \mathrm{C}$ to $93{ }^{\circ} \mathrm{C}$, and for relative humidity ranging from $2 \%$ to $75 \%$. [Work supported by the Department of Energy and the Institute of Paper Science and Technology.]

\section{2:30}

2pEA5. A physically attractive alternative to Lighthill's quadrupole aerodynamic sound source. Alan Powell (Dept. of Mech. Eng., Univ. of Houston, TX 77204-4006)

Now an impressed force/unit vol. $f_{i}$ acting alone on an acoustic medium results either directly in the formal mathematical monopole distribution $\partial f_{i} / \partial y_{i}$ but which is physically unrealistic, not appearing in the continuity equation, or the given dipole distribution $f_{i}$ by the divergence theorem. These both yield point dipoles, $\mathcal{I} y_{k} \partial f_{i} / \partial y_{i}$ by Taylor expansion or the expected $\mathcal{I} f_{i}$, where $\mathcal{I}=$ "of strength proportional to the instantaneous volume integral of." Aerodynamic sound generation based on Lighthill's inhomogeneous wave equation yieds directly a source distribution of the form (a) monopole $\partial^{2} T_{i j} / \partial y_{i} \partial y_{j}, \approx \partial^{2} \rho u_{i} u_{j} / \partial y_{i} \partial y_{j}=\partial / \partial y_{i}$ - $\partial \rho u_{i} u_{j} / \partial y_{j}$. By the divergence theorem alternative integrally equal distributions are (b) dipole $\partial \rho u_{i} u_{j} / \partial y_{j}$ and (c) Lighthill's quadrupole $\rho u_{i} u_{j}$. All yield point quadrupoles for (a) $\mathcal{I} y_{k} y l \partial^{2} \rho u_{i} u_{j} / \partial y_{i} \partial y_{j}$ and (b) $\mathcal{I} y_{k} \partial \rho u_{i} u_{j} / \partial y_{i}$ by Taylor expansions, (c) $\mathcal{I} \rho u_{i} u_{j}$. Distribution (a) is unrealistic physically just like $\partial f_{i} / \partial y_{i}$; (b) appears directly in the momen-
$3: 15$

2pEA7. The performance of two-dimensional acoustic resonators with large openings. K. Scott Line, Michael J. Anderson, and Ralph S. Budwig (Dept. of Mech. Eng., Univ. of Idaho, Moscow, ID 83844-0902)

We address the design of acoustic resonators with large openings. This problem is of interest to those who wish to achieve high levels of acoustic amplification in spite of energy leakage through the openings. A 2-D analytical model was used to predict the performance of a rectangular resonator with rigid walls and compare the performance to the corresponding situation for a cavity between two rigid semi-infinite walls. The fluid in the resonator was air, and the dissipation model included body absorption and simplified boundary layer losses. It was found that the collimation of the transducer field, and the nature of the attenuation model determined the performance of a resonator with large openings. For the dissipation model used, it was predicted that optimal performance of a resonator with openings would be obtained at a transducer $k a$ of approximately 100. Experimental measurements of closed and open cavity quality factors, for transducer $k a$ ranges lower than 100 , showed the same trends as analytical predictions, however, the measured quality factors were much lower than the analytical predictions.

\section{3:30}

2pEA8. Inflatable lightweight low-frequency loudspeaker. Robert Corsaro (Code 7135, Naval Res. Lab., Washington, DC 20375)

In an aerospace application we required a low-mass sound generator with good performance at low audio frequencies (i.e., 30-300 Hz). One approach which has been successful in meeting these needs makes use of an inflatable tube. The actuation component is a "Thunder" actuator (Face Intl. Corp.), selected for its high displacement and fast response. Conventionally, when coupled to loudspeaker diaphragms these actuators have failed to produce the combination of force and displacement needed for low-frequency sound generation. In the present device the actuator is coupled instead to a flat, compliant diaphragm supported on the edges by an inflatable tube. Sound pressure levels measured in the farfield $(25 \mathrm{~cm})$ using only $200 \mathrm{~V}$ peak drive (one-third or its rating) we found nominal levels of $746 \mathrm{~dB}$ over the band from 38 to $330 \mathrm{~Hz}$. In the uninteresting region above $330 \mathrm{~Hz}$ the output is somewhat higher, but remains well behaved to at least $2 \mathrm{kHz}$. [Work supported by the NRL/ONR Smart Blanket program, which is exploring the use of active controlled surface covers for reducing sound levels in payload fairing regions.] 
Session 2pMU

Musical Acoustics: Caribbean Musical Instruments and Traditions

\author{
Paul A. Wheeler, Cochair \\ 1595 North 1600 East, Logan, Utah 84341 \\ Thomas D. Rossing, Cochair \\ Physics Department, Northern Illinois University, DeKalb, Illinois 60115 \\ Chair's Introduction-1:00 \\ Invited Papers
}

1:05

2pMU1. Overview of Caribbean music. Martha Ellen Davis (School of Music, Univ. of Florida, 1350 Turlington, Gainesville, FL 32611)

To frame the 2001 meeting of the ASA within the soundscape of the region, this paper offers an overview of the diversity of musical sounds and sound-makers, i.e., instruments, of the Caribbean and Circum-Caribbean region. Many are in fact present in the Miami-Dade/Fort Lauderdale-Broward area because of the growing presence of immigrant populations, as well as the influence of some of their traditions on "world beat" music (the subset of "world music" with African influence). The survey of sounds of the Caribbean, illustrated audiovisually, will include both the commercially disseminated genres ("popular music"), and others which are not marketed or marketable, although in some cases the roots of popular music. The "unknown" genres include social dances, as well as work songs, sacred drumming, ritual songs, and more. The paper proposes African culture influence as the common denominator which musically unifies the region and explains why.

\title{
$1: 35$
}

2pMU2. Traditional Junkanoo music instruments of the Bahamas. Nina Wood (P.O. Box 15864, Plantation, FL 33318-5864)

Junkanoo is a carnival-like parade that takes place twice a year in the Bahamas. The parade grew out of the music practices of Africans who had been enslaved in the Bahamas during the nineteenth century. Today, the parade incorporates large, elaborate costumes, and music played on traditional instruments. The number of participants in Junkanoo has grown tremendously, and moves have been made to incorporate amplified, electronic music in the parade in order to project the volume of the music over the sounds of the participants and spectators. Despite attempts to incorporate amplified music, however, acoustic instruments have remained primary. Junkanoo music performance has retained many of its African characteristics in terms of timbre, percussion, and density, and throughout the past two decades adjustments have been made to the instruments to enhance these characteristics. In this discussion the instruments of the Junkanoo music tradition will be introduced, their timbral qualities will be examined, and their particular functions and relationships within the tradition will be explored.

2:05

2pMU3. Science of steelpans: What is known and what is not. Thomas D. Rossing (Phys. Dept., Northern Illinois Univ., DeKalb, IL 60115) and Uwe J. Hansen (Indiana State Univ., Terre Haute, IN 47809)

One of the most important musical instruments developed in the 20th century is the Caribbean steelpan. In addition to being the foremost musical instrument in its home country of Trinidad and Tobago, the steelpan is becoming increasingly popular in North America and Europe. Scientific studies on the instrument have not kept pace with its growing popularity, however. This paper reviews some of the scientific studies that have been made on steelpans in our laboratory and elsewhere. One of the most important steps in understanding the steelpan is to understand how it vibrates and produces sound. We report measurements of modal shapes in different steelpans by prominent makers and attempt to interpret these results in terms of the geometry and fabrication history of these pans. We also discuss the physics of the material, including the residual stresses, the surface hardness, and the effect of heating.

2:35

2pMU4. Modal analysis of steelpans: Holographic, computer, sand patterns, and microphone scannning. Uwe J. Hansen (Dept. of Phys., Indiana State Univ., Terre Haute, IN 47809) and Thomas D. Rossing (Dept. of Phys., Northern Illinois Univ., DeKalb, IL 60115)

We compare several methods for modal analysis that have been applied to Caribbean steelpans. Holographic interferometry offers the best spatial resolution of operating deflection shapes, and hence of normal modes. Recording holographs on photographic film tends to be rather time consuming, however. Electronic TV holography, on the other hand, offers the opportunity to observe vibration 
motion nearly in real time. Striking a steelpan with an instrumented force hammer excites many modes at once, and a computer program is used to determine the normal modes by means of a curve-fitting program. In the oldest method of modal analysis (and still a useful one), a fine powder is sprinkled on the surface of a steelpan vibrating at the frequency of a given mode. The powder collects at the nodal lines, providing a Chladni pattern or map of that mode.

\section{3:05-3:15 Break}

2pMU5. The Steelpan Development Centre at The University of the West Indies. Brian Copeland, Clément Imbert, and Derek Gay (Faculty of Eng., The Univ. of the West Indies, St. Augustine, Trinidad and Tobago, West Indies)

This paper describes the Steelpan Development Centre (SPDC) of The University of the West Indies (UWI), a specialist center created to explore, support, and develop all aspects of the technology of the steelpan. The SPDC places special emphasis on the development on steelpan manufacturing technology and performance in the Caribbean region. While the major focus of the center is pan technology, its mission also includes consideration of other aspects that impact on the growth of the instrument, in collaboration with the UWI Center for Creative and Festival Arts. In particular, the SPDC has strong interest in providing technical, business, and administrative support to the many regional tuners who, by and large, still operate at the craft level. The paper looks at the motivation for the formation of the center, a few of the developments to date and some of the projects listed for consideration.

\section{$3: 45$}

2pMU6. Materials and manufacturing of steelpans. C. A. C. Imbert (Steelpan Development Ctr., Faculty of Eng., The Univ. of the West Indies, St. Augustine, Trinidad and Tobago, West Indies)

The musical instrument referred to as the Caribbean steel drum (called the steelpan or simply pan by true aficionados) is traditionally hand-crafted from 55 U.S. gallon oil drums. These drums are made to satisfy certain physical and mechanical properties but certainly not for musical instruments. As a result pan builders/tuners have to reject a relatively large number of drums because they do not respond well enough to produce fine quality lead instruments. In this paper the results of the mechanical/metallurgical investigation of several drum steels are reported — steels that respond well and others that do not—as well as the process of hardening steelpans by nitriding. Also reported are the results of mechanized methods of sinking the pan compared to the traditional manual process.

\section{Contributed Paper}

\section{4:15}

2pMU7. Sound radiation from Caribbean steelpans. Brian Copeland (Dept. of Elec. and Computer Eng., Univ. of the West Indies, St. Augustine, Trinidad), Andrew Morrison, and Thomas D. Rossing (Phys. Dept., Northern Illinois Univ., DeKalb, IL 60115)

Besides radiating sound from the note area being struck, a steelpan radiates from neighboring note areas that vibrate sympathetically, from the areas between notes, and from the skirt [Rossing et al., Phys. Today 49, 24-29 (1996)]. We have used a two-microphone intensity probe to measure the sound field around steelpans when various note areas are excited with swept sine wave force in an anechoic room. The radiation patterns in planes parallel and perpendicular to the surface of the pan are plotted and analyzed. 
Session 2pNS

\title{
Noise: Noise Measurement, Sources, Control
}

\author{
Joseph Pope, Cochair \\ Pope Engineering Company, P.O. Box 236, Newton, Massachusetts 02459-0002 \\ Bennett M. Brooks, Cochair \\ Brooks Acoustics Corporation, 27 Hartford Turnpike, Vernon, Connecticut 06066
}

Contributed Papers

$1: 30$

2pNS1. Directivity of the diffracted field around a half-plane. Jeong Ho You, Penelope Menounou, and Wayne M. Wright (Dept. of Mech. Eng., Univ. of Texas, Austin, TX 78712)

The directivity of the diffracted field around a half-plane barrier is investigated. Experiments were carried out in air using a spark source generating $\mathrm{N}$-shaped pulses, a capacitor microphone, and a rigid aluminum plate with: (i) straight top edge (reference case), (ii) jagged top edge, and (iii) straight edge covered with sound absorptive material. Measurements were taken around the plate by varying the angular position of the microphone while keeping its radial distance from the barrier edge constant. The following were observed: (i) Straight edge: The diffracted pulse changes polarity as it passes across the two shadow boundaries and the continuation of the barrier in the half-space above it. (ii) Jagged edge: Although jaggedness in the top edge of the barrier drastically changes the shape of the diffracted pulse, the directivity pattern remains roughly the same. (iii) Sound absorptive edge: Although the shape of the diffracted pulse remains the same as in the reference case, the directivity patterns change considerably and changes are more pronounced at the source side. The experimental data and observations were in good agreement with theoretical predictions from the Directive Line Source Model [Menounou et al., J. Acoust. Soc. Am. 107, 103-111 (2000)]. [Work supported by Texas ATP.]

\section{$1: 45$}

2pNS2. Feasibility of a phased acoustic array for monitoring acoustic signatures from meshing gear teeth. Adrian A. Hood and Darryll J. Pines (Univ. of Maryland, College Park, MD 20143, djpterp@eng.umd.edu)

This research investigates the feasibility of sensing damage emanating from rotating drive train elements such as bearings, gear teeth, and drive shafts via airborne paths. The use of a planar phased acoustic array is evaluated as a potential fault detection scheme for detecting acoustic signatures radiating from gearbox components. Specifically, the use of beam focusing and steering to monitor individual tooth mesh dynamics are analyzed, taking into consideration the constraints of the array/gearbox geometry and the spectral content of typical gear noise. Experimental results for a linear array are presented to illustrate the concepts of adaptive beam steering and spatial acoustic filtering. Also, a comparison is made between the radiated spectrum of a damage free system and one with seeded faults using spur gears. This feasibility study indicates that the planar array can be used to track the acoustic signatures of gear mesh dynamics of typical helicopter systems at higher harmonics of the mesh frequency.

2:00

2pNS3. Machine vision improves sound level meter calibration. Guillermo Arcas, Mariano Ruiz, and Raul Pagan (Universidad Politecnica de Madrid, INSIA Crta. Valencia Km-7, Madrid 28031, Spain)

In this paper, important improvements in sound level meter (SLM) calibration are presented. One of the most important problems that the calibration laboratories are facing today is the long time required for calibrating certain SLMs. Instruments without a dc output require that an operator reads the SLM response in every test. This makes the process slow and tedious, and induces errors due to the operators fatigue. By using an image acquisition card with a low cost analog camera to acquire an image of the sound level meters display, calibration time can be reduced significantly. Once an image of the SLMs display has been acquired, machine vision techniques (OCR) are used to recognize the sound level meter indication. This process reduces calibration time and errors produced by operators fatigue.
2pNS4. The software architecture for an analyzer sound level meter with DSP-bios. Juan M. Lopez Navarro, Mariano Ruiz Gonzalez, Manuel Recuero Lopez, and Raul Pagan Muoz (INSIA Ctra. de Valencia Km 7, Madrid 28031, Spain)

This paper shows the software architecture implemented for the accomplishment of an analyzer sound level meter in one-third octave based on a Digital Signal Processor TMS320C6701 of Texas Instruments. In order to minimize the cost of the equipment, it has been done without the elements of interface with the user (keyboard and displays). For the operation and control of the equipment a laptop, or a PDA may be used. A communication RS232C is used in the local way; and in a remote way, a connection through built-in MODEM GSM. The sever application is executed in the signal processor. It has been implemented in $\mathrm{C}$ language and it runs on an real time operating system (DSP-Bios provided by Texas Instruments). DSP-Bios provides the management of events, queues, and timings. The application has the necessary algorithms to make the measures and sends the results to the visualizer, or to a center of remote operation by means of the GSM. The client application that is executed in the visualization element (Laptop or PDA) is developed in Visual Basic. This application can run on any Windows operating system depending on the terminal used: Windows 9x or Windows 2000 for portable ones and on Windows CE for mobile ones. 
2pNS5. Determination of reference sound source sound power levels using ARI Standard 250. Stephen J. Lind (The Trane Co., 3600 Pammel Creek Rd., La Crosse, WI 54601)

The Air Conditioning and Refrigeration Institute (ARI) Standard 250 Performance and Calibration of Reference Sound Sources was used to establish one-third octave band sound power levels of several reference sound sources (RSS). The work was carried out in an anechoic chamber that was qualified at frequencies of $100 \mathrm{~Hz}$ and above using ANSI S12.35. Measurements were made with circular slices around the RSS at heights from $5 \mathrm{~cm}$ to $195 \mathrm{~cm}$ in $10 \mathrm{~cm}$ increments using both intensity and sound pressure. A 19 point fixed array was also employed for comparison of one of the sources. Sound power levels were compared from the $50 \mathrm{~Hz}$ to the $10000 \mathrm{~Hz}$ one-third octave bands using several methods. The results are compared to the sound power levels provided by the manufacturer.

\section{2:45-3:00 Break}

3:00

2pNS6. Numerical prediction of noise generated from low-pressure axial-flow fans. Li Song and Wang Dongbao (Dept. of Eng. Mech., Tsinghua Univ., Haidian District, Beijing 100084, PROC)

Low-pressure axial-flow fans are widely used in ventilation, air conditioning, and cooling, but it is very difficult to predict their noise. In 1993 Lee et al. predicted the noise level of low-pressure axial fans according to a wake-flow shedding model based on the mechanism that the noise is induced by a lift force pulse caused by wake-flow vortex shedding [J. Sound Vib. 164, 327-336 (1993)]. The main approximation that results in considerable errors is that the parameters of a flat-plate boundary layer at the trailing edge are adopted instead of that of a blade boundary layer. In this paper the parameters of the boundary layer, including the distribution of nominal thickness and momentum thickness of boundary layer along the blade height, are obtained by numerical simulation of the 3-D turbulent flow field in axial-flow fans. It is shown from the predicted results for two model axial fans that the noise level agrees with measured data quite well. The errors are 2-4 dB for A-weighted sound pressure level (SPL) and 3-4 $\mathrm{dB}$ for linear SPL. Furthermore the effects of parameters of the blade boundary layer on noise level and spectrum are also discussed in more detail in this paper.

\section{3:15}

2pNS7. Source models for turbulence generated sound in ducts. Mats Abom (MWL, Dept. of Vehicle Eng., KTH, 10044 Stockholm, Sweden)

Turbulence or flow generated sound is a problem in many duct and pipe systems. This type of noise will add to the primary noise from fluid machines (e.g., fans) connected to the system and often sets the limit for the sound attenuation possible. For low Mach numbers the flow generated noise will primarily be associated with unsteady flow separation occurring, e.g., at constrictions and bends, resulting in a dipole type of source. In this paper a procedure for deriving source models for turbulence generated sound in the low-frequency plane wave range is described. The source models are formulated as active acoustic 1- and 2-ports [H. Boden and M. Abom, Acta Acustica 3, 549-560 (1995)], which makes them suitable for implementation in software for analysis of duct networks. Regarding the active part or the source strength, it can be obtained using a semiempirical model for flow separation noise [P. A. Nelson and C. L. Morfey, J. Sound. Vib. 79, 263-289 (1981)]. The passive part can be well approximated in the low-frequency limit by a quasi-stationary model derived from the conservation of mass and momentum in the flow. Examples of tests and validation of the proposed model are also presented.
2pNS8. Broadband passive noise control in air/water using structural acoustic silencers. Sripriya Ramamoorthy, William P. Cook, Karl Grosh (Dept. of Mech. Eng., Univ. of Michigan, 2350 Hayward Ave., Ann Arbor, MI 48109-2125), and Tony G. Nawar (Dept. of Elec. Eng. and Computer Sci., Univ. of Michigan, 1301 Beal Ave., Ann Arbor, MI 48109-2122)

The effectiveness of introducing flexible structural layers into fluid and air conveying ducts for controlling noise is investigated through theoretical and experimental means. Previous work has shown that using flexible rather than rigid walls can theoretically achieve high transmission losses. However, for hydraulic applications these relatively thin layers cannot sustain the high static pressures seen in real situations and for pneumatic applications, radiated noise from these walls (called breakout noise) is a significant issue. Designs that introduce a second duct below the flexible wall region of the main duct allows for equilibration of the static pressure and elimination of breakout noise. However, plane waves can now propagate through the duct without exciting the plate, thus reducing the effectiveness of the device. Designs suitable for hydraulic/pneumatic applications, that overcome these issues and achieve appreciable transmission loss are investigated. Results based on 2.5 D finite element simulations will be compared with experimental results. [Research funded by ONR and NSF.]

\section{$3: 45$}

2pNS9. The effect of active noise control actuator placement on the total sound power radiated by modally vibrating cylinders and plates. Jennifer L. Cooper and Jiri Tichy (Grad. Prog. in Acoust., The Penn State Univ., University Park, PA 16801)

Many practical radiators can be modeled as vibrating bars or plates. The radiation caused by their modal vibration is studied replacing them by point sources at each antinodal location. Active control of their radiated sound power is investigated using point secondary sources. The maximum obtainable attenuation is examined as a function of the distance from primary to secondary sources and the separation of secondary sources. For a given distance from the primary sources, the obtained attenuation is independent of secondary source separation for a range of wavelengths. For larger secondary source separations, the control can be simplified, allowing each secondary source to be controlled by only one microphone, as opposed to the usual method of controlling all secondary sources by all the microphones. 


\title{
Session 2pPAa
}

\section{Physical Acoustics: High Amplitude Effects in Resonators II}

\author{
Anthony A. Atchley, Cochair \\ Graduate Program in Acoustics, Pennsylvania State University, P.O. Box 30, Applied Science Building, \\ State College, Pennsylvania 16804 \\ Victor W. Sparrow, Cochair \\ Graduate Program in Acoustics, Pennsylvania State University, P.O. Box 30, Applied Science Building, \\ State College, Pennsylvania 16804
}

\section{Invited Papers}

\section{1:00}

2pPAa1. Acoustic agglomeration experiments and modeling. G. Douglas Meegan, Chris E. Peterson, and Ronald E. Kumon (Appl. Res. Labs, Univ. of Texas, Austin, TX 78713-8029)

Acoustic agglomeration of aerosols has been known since at least 1931 when it was observed that particles tend to "stick" together in the presence of an intense acoustic field, thereby forming larger particles. Since the 1970s acoustic agglomeration has been an active area of research because of its potential applications for the control of particulate emissions resulting from fossil fuel combustion. In this talk, experimental methods used in the past to study acoustic agglomeration are described, along with improvements we have introduced. A simple standing wave tube is one of the devices used in our laboratory to measure the dependence of acoustic agglomeration on sound pressure level, frequency, and exposure time. Results are presented and compared with theoretical predictions based on existing models. The potential application to diesel engine emissions and the filtration of hazardous particulate will be discussed.

1:30

2pPAa2. A computational study of acoustic streaming in a duct. William R. Erskine and Ahmet Selamet (Dept. of Mech. Eng., The Ohio State Univ., Columbus, OH 43210)

The present work is a continuing investigation of Rayleigh streaming, which is a nonlinear phenomenon characterized by acoustically driven flows attenuated by viscous effects due to the presence of walls, resulting in nonzero average streaming velocities and circulation zones within a duct. A 2-D long and narrow duct is considered with one end closed and the other end subject to a sinusoidal acoustic velocity at quarter-wavelength frequencies and varying amplitudes, leading to a standing wave within the duct. A computational fluid dynamics code based on the Pressure Implicit with Split Operators (PISO) algorithm and finite volume method is used to solve the unsteady, compressible Navier-Stokes equations with turbulence modeling. Since the formation of streaming depends on the velocity profile within the Stokes layer, the grid resolution is increased near the walls. The effect of inlet conditions is studied in terms of circulation patterns, including the number of zones per wavelength, penetration depth into the fluid, and mean velocity profiles across the boundary layer. Some of the computational predictions are also compared with the classical analytical treatments.

\section{Contributed Papers}

\section{2:00}

2pPAa3. Shock-free, forced oscillations of an air column in a closed tube. Nobumasa Sugimoto, Takeshi Hashiguchi (Dept. of Mech. Sci., Grad. School of Eng. Sci., Univ. of Osaka, Toyonaka, Osaka 560-8531, Japan), and Mitsuhiro Masuda (Dept. of Thermal Energy Systems, Ecology and Energy Systems Res. Ctr., Sanyo Electric Co., Hashiridani, Hirakata, Osaka 573-8534, Japan)

This paper reports generation of high-amplitude, shock-free oscillations of an air column in a closed tube of uniform cross section. The air column is driven sinusoidally by the bellows mounted at one end of the tube at a frequency equal or close to the resonance frequency of the lowest mode. The shock-free oscillations are achieved by connecting an array of Helmholtz resonators to the tube axially. The array of resonators gives rise to wave dispersion, which changes the sound speed in this tube to depend on the frequency and makes the tube dissonant by avoiding coincidence of the higher-harmonic frequencies of excitation and the resonance ones of the higher modes. Each resonator is identical and small in a sense that the cavity's volume is chosen much smaller than the tube's volume per axial spacing between neighboring resonators, while the natural frequency of the resonator is chosen even higher than the lowest resonance frequency.
Frequency response is obtained and compared with the theory up to the resonance frequency of the third mode. It is observed that the shock-free oscillations are realized at the sound pressure level of $170 \mathrm{~dB}$ where the shock would emerge unless the array were connected.

\section{2:15}

2pPAa4. Suppression of nonlinear energy decrease by linear absorption and enhancement of resonant properties of nonlinear systems. Oleg Rudenko (Dept. of Acoust., Faculty of Phys., Moscow State Univ., Moscow 119899, Russia, rudenko@acs366b.phys.msu.su)

A general question of nonlinear vibration and wave theory is discussed which is of special interest for nonlinear acoustics and technologies. Examples of nonlinear systems of different physical nature are given to illustrate how the properly introduced additional absorption can decrease the nonlinear losses and improve the resonant properties of nonlinear systems. Both traveling and localized standing waves are considered. The possibilities to enhance the Q-factor of acoustic resonators are discussed in detail, as well as links to the turbulence, solid state physics, and electronics. [Work supported by CRDF, INTAS, and RFBR grants.] 


\title{
Session 2pPAb
}

\section{Physical Acoustics: Thermoacoustics}

\author{
Richard Raspet, Chair \\ National Center for Physical Acoustics, University of Mississippi, 1 Coliseum Drive, University, Mississippi 38677
}

\section{Contributed Papers}

\section{2:45}

2pPAb1. Performance of a thermoacoustic prime mover without a stack. Stefan J. Turneaure, Ray Scott Wakeland, and Robert M. Keolian (Grad. Prog. in Acoust., Penn State Univ., P.O. Box 30, State College, PA 16804)

A standing-wave thermoacoustic prime mover which does not contain a stack has been constructed and tested. The device consists of two heat exchangers and a resonator body. The cold heat exchanger may be moved relative to the hot heat exchanger while the resonator is sealed and thermoacoustically generated sound is present. The device has a crosssectional area of about $500 \mathrm{~cm}^{2}$ and operates near $600 \mathrm{~Hz}$ with ambient air as the working gas. It will be explained how a no-stack thermoacoustic device may in principle have a greater efficiency than a stack-based device. In contrast to the well understood stack-based standing wave thermoacoustic engines, it is expected that the efficiency for a no-stack device will be nearly proportional to the acoustic pressure amplitude. Preliminary measurements on the device have achieved acoustic pressure amplitudes of $2.5 \%$ of the mean pressure. An interesting result is the presence of spontaneous thermoacoustic oscillations even when the gap between the cold and hot heat exchangers is several times larger than the gas displacement, $2 x_{1}$. [Work supported by ONR.]

\section{3:00}

2pPAb2. Investigation of stack materials for miniature thermoacoustic engines. Ehab Abdel-Rahman, Young Kwon, and Orest G. Symko (Dept. of Phys., Univ. of Utah, Salt Lake City, UT 84112-0830, ehab@physics.utah.edu)

In a thermoacoustic engine one of the most critical components is the stack that interacts thermally with a sound field. Its effective surface area determines directly the cooling power and efficiency while the thermal properties determine the losses. Moreover, as the engines are miniaturized, stack performance on a small scale becomes a limiting factor, especially when a stack length of $1000 \mu$ or less has to maintain temperature differences of around $30^{\circ} \mathrm{C}$. We have investigated as stack material cotton wool, glass wool, ceramics, silica aerogel, carbon fibers, and carbon powder by measuring flow resistance at high acoustic frequencies, $4-20 \mathrm{kHz}$, and thermal transport properties. The mechanical properties and ease of machining to small dimensions, of the order of $1 \mu$, have been studied. In selecting the ideal material for very small devices, a compromise needs to be made between thermoacoustic and mechanical properties. [Work supported by ONR.]

\section{$3: 15$}

2pPAb3. Experimental study of a thermoacoustic electrical generator. Jin Liu, Philip S. Spoor, John A. Corey (CFIC, Inc., Troy, NY 12180), and Steven L. Garrett (Penn State Univ., State College, PA 16804)

The stack and heat exchangers from the historic Five Inch Engine [G. W. Swift, J. Acoust. Soc. Am. 92, 1551 (1992)] have been joined to a 2.02-m long resonator tube terminated by a variable acoustic load [A. M. Fusco et al., J. Acoust. Soc. Am. 91, 2229 (1992)]. Operation in 20-bar pure helium delivered $160 \mathrm{~W}$ to the load with an electrical heat input of $2.76 \mathrm{~kW}$, producing a hot-end external duct temperature of $655^{\circ} \mathrm{C}$. Applied heat measuring $1.41 \mathrm{~kW}$ was exhausted through the cold exchanger to a water stream at $25^{\circ} \mathrm{C}$, with the remaining power producing useful work or otherwise lost by conduction through the engine and to the surroundings. Measured acoustic pressure amplitudes for electrical heat inputs in the range from 348 to $2773 \mathrm{~W}$ agreed with the DELTAE model to within $12 \%$, but the error in the hot-end temperature prediction was $25 \%$. The engine was then attached to a CFIC Model B-300 electrodynamic motor/alternator to explore feasibility of distributed power generation and co-generation. [The loan of the 5-in. engine, by G. W. Swift, Los Alamos National Laboratory, is gratefully acknowledged. J.L. and S.L.G. were supported, in part, by ONR.]

3:30

2pPAb4. Wet-walled thermoacoustics. William Slaton and Richard Raspet (The Natl. Ctr. for Physical Acoust., The Univ. of Mississippi, University, MS 38677)

An analytic solution of sound propagation in wet-walled tubes with a temperature gradient will be presented. The tube contains an inert gasvapor mixture with a thin layer of condensed vapor coating the tube wall. The vapor phase condenses and evaporates from this layer during an acoustic cycle. This phased evaporation and condensation modifies traditional energy density and wave number equations. It is found that mass and heat transport act in parallel thereby increasing the acoustic energy density for proper choice of gas mixtures. This increase in energy density due to inert gas-vapor working fluids can be quite large and even dominate the traditional thermal transport contribution. The use of phased evaporation-condensation in gas mixtures provides a path to higher energy density in thermoacoustic systems. The effects on sound propagation, energy density, and efficiency due to the presence of an inert gas-vapor working fluid in tubes with wet walls will be discussed. Also, computational modeling of thermoacoustic refrigerators utilizing an inert gasvapor working fluid will be presented. Modeling is done using DeltaE software with modifications made to explore inert gas-vapor mixtures. Design of a practical thermoacoustic refrigerator utilizing wet-walled thermoacoustics will be presented. [Work supported by ONR.]

\section{$3: 45$}

2pPAb5. Reduced onset temperature difference in wet thermoacoustic engines. William V. Slaton, John W. Rayburn, Robert A. Hiller, and Richard Raspet (Natl. Ctr. for Physical Acoust., Univ. of Mississippi, University, MS 38677)

Measurements of the onset temperatures of a standing-wave thermoacoustic prime mover in air show a dramatically decreased onset temperature difference when liquid water is present on the stack surfaces. These data may be explained by considering the condensation and evaporation of 
water during the acoustic cycle [W. V. Slaton and R. Raspet, J. Acoust. Soc. Am. 108, 2569 (2000)]. The inert gas-vapor mixture thermoacoustic propagation equations are integrated through the resonator/stack system to determine the quality factor and resonant frequency using complex frequency analysis. In addition the 1-D thermoacoustic modeling code DELTAE was modified to include these effects in the stack to predict onset temperatures.

\section{4:00}

2pPAb6. The use of parallel-plate regenerators in thermoacousticStirling engines. Scott Backhaus and Gregory W. Swift (Condensed Matter and Thermal Phys. Group, Los Alamos Natl. Lab., Los Alamos, NM 87545)

The regenerator is a key element in a thermoacoustic-Stirling engine. Typically, it is made by stacking up many layers of fine-mesh stainlesssteel screen. With a high heat capacity and a typical pore size much smaller than the thermal penetration depth, it provides a nearly isothermal environment at the acoustic frequency. By almost eliminating the temperature oscillations of the gas in it, the regenerator minimizes the unwanted enthalpy flux from the hot heat exchanger to ambient heat exchanger and keeps the engine's efficiency high. However, the small pore size also causes a significant amount of viscous dissipation, which lowers the efficiency. A regenerator made of parallel plates can be designed to provide the same heat transfer properties as a screen regenerator with much lower viscous dissipation. By replacing a screen based regenerator with a carefully constructed parallel-plate regenerator, the acoustic power output of a thermoacoustic-Stirling engine is nearly doubled, with significant increases in efficiency at high amplitudes. [Work funded by the Office of Basic Energy Sciences within the U.S. DOE.]

\section{4:15}

2pPAb7. One-dimensional acoustic streaming instabilities in traveling-wave thermoacoustic devices employing jet pumps. Scott Backhaus and Gregory W. Swift (Condensed Matter and Thermal Phys. Group, Los Alamos Natl. Lab., Los Alamos, NM 87545)

Traveling-wave thermoacoustic devices employ a Stirling-type thermodynamic cycle and thus enjoy an inherent efficiency advantage over standing-wave devices. However, if the regenerator of the traveling-wave device is embedded in a loop, acoustic streaming around the loop can cause a heat leak that greatly reduces the efficiency. In several travelingwave devices, asymmetric nozzles, or synthetic-jet pumps, have been successfully used to suppress the streaming and, therefore, the heat leak. However, in one traveling-wave refrigerator, an instability that precludes streaming suppression is driven by the interaction between the syntheticjet pump and the thermoacoustic system. Calculations showing how this instability arises will be presented. [Work funded by the Office of Basic Energy Sciences within the U.S. DOE.]
2pPAb8. A study of thermoacoustic instabilities in a resonator with heat release. Konstantin I. Matveev and Fred E. C. Culick (Caltech, 301-46, Pasadena, CA 91125)

Excitation of acoustic modes in systems with heat release is a complex process, involving coupling between the heat transfer rate and the acoustic pressure. The Rijke tube is the simplest device for studying the fundamental principles of thermoacoustic instabilities both experimentally and theoretically. In our investigation, special attention is paid to reproducibility and precision of the measurements. Experimental results for transition to instability are obtained when controlled parameters (flow rate, heater position, and supplied power) are varied in a quasisteady fashion. The stability boundaries exhibit hysteretic behavior not previously reported. Properties of the limit cycles in the region of instability have also been determined. A mathematical model is developed that incorporates heat transfer, acoustics, and thermoacoustic interactions. An energy balance, accounting for all types of heat transfer, allows for the determination of temperature variations and heat flux along the tube. The acoustic modes, generally nonorthogonal, corresponding to particular experimental thermal conditions, are computed. Solutions of the linearized wave equation with both loss and heat source terms determine the stability of acoustic modes. Hysteresis effects at the stability boundary and amplitudes of the limitcycles are calculated from the nonlinear model.

\section{$4: 45$}

2pPAb9. Saturation of the thermoacoustic separation of a binary gas mixture. Drew A. Geller and Gregory W. Swift (Condensed Matter and Thermal Phys. Group, Los Alamos Natl. Lab., Los Alamos, NM 87545)

Spoor and Swift have previously shown [Phys. Rev. Lett. 85, 16451649 (2000)] that sound waves can produce the separation of a binary mixture of gases in a duct. This phenomenon is caused by the conspiracy of two effects at the thermoviscous boundary layer: (i) oscillating temperature gradients drive thermal diffusion perpendicular to the direction of sound propagation; and (ii) viscosity sets up a time-averaged counterflow process. In order to assess the relevance and potential uses of this mechanism, it is important to determine the concentration gradient at which the separation process saturates. Here, it is shown that the saturation is determined primarily by ordinary diffusion and by a remixing flux due to the oscillatory motion. Both of these remixing processes are proportional to the concentration gradient, but only the latter process depends on the acoustics, being proportional to the squared volume flow rate and inversely proportional to the frequency. For $\mathrm{He}-\mathrm{Ar}$ mixtures, saturation concentration gradients near $10 \% / \mathrm{m}$ have been achieved, in agreement with detailed calculations of these processes. Evidence to date supports the possibility of more challenging separations, e.g., of isotopes. [Work supported by the DOE Office of Basic Energy Sciences.] 
Session 2pPP

\title{
Psychological and Physiological Acoustics: Localization, Pitch, Hearing Aids, Instrumentation and Other Topics (Poster Session)
}

\author{
Constantine Trahiotis, Chair \\ Surgery and Neuroscience, University of Connecticut Health Center, L4071, Farmington, Connecticut 06032
}

\section{Contributed Papers}

\begin{abstract}
All posters will be on display from 1:30 p.m. to 5:00 p.m. To allow contributors an opportunity to see other posters, contributors of odd-numbered papers will be at their posters from 1:30 p.m. to 3:15 p.m. and contributors of even-numbered papers will be at their posters from 3:15 p.m. to 5:00 p.m.
\end{abstract}

2pPP1. The effects of presentation time on the localization accuracy of 3D sound through headphones. Fang Chen and Jonas Sääv (Linköping Univ. IKP/IAV, SE-581 83 Linköping, Sweden, fanch@ikp.liu.se)

The present study deals with the relationship between sound presentation time and localization accuracy of 3D sound presented through headphones. Huron Lake CP4 system was used for sound presentation and Flock of birds tracking system was used to register movement of the head. There were 31 volunteers ( 15 females and 16 males) in this experiment. Six different types of sounds were presented with the duration of $0.5,2,4$, and $6 \mathrm{~s}$. They were dog bark $(0-500 \mathrm{~Hz})$, coin drop with three different bandwidths $(0-1650 \mathrm{~Hz}, 1650-6500 \mathrm{~Hz}$, and $0-8500 \mathrm{~Hz})$, alarm $(0-5500$ $\mathrm{Hz})$ and male speech voice $(0-7000 \mathrm{~Hz})$. The results showed that the accuracy of localizing the sound is significantly related to the duration of the sound presentation. The perceived azimuth difference for $0.5 \mathrm{~s}$ duration $\left(45^{\circ} \pm 15^{\circ}\right.$ in average) is significantly higher compared with the longer presentation duration. The perceived azimuth difference for $2 \mathrm{~s}$ duration is also significantly higher than 4 and $6 \mathrm{~s}$ duration $\left(21^{\circ} \pm 9^{\circ}\right.$ in average). There is not any significant difference between 4 and $6 \mathrm{~s}$ duration. There is not any significant difference between male and female subjects, but individual difference is significant.

2pPP2. The effect of pinna protrusion angle on localization of virtual sound in the horizontal plane. Navarun Gupta, Carlos Ordonez, and Armando Barreto (Florida Intl. Univ., Dept. of Elec. and Computer Eng., 10555 W. Flagler St., Miami, FL 33174)

The transformation of a sound from its origin to each of the eardrums of a listener, due to the head, torso, and outer ear of the listener, are modeled by the so-called head-related transfer functions (HRTFs) for each individual and each location around the listener. These HRTFs can be used to transform a monoaural sound into left and right sounds that will give the subject the illusion of a virtual sound placement, at the location associated with the HRTF used. However, subjects tend to confuse sounds virtually placed in the front hemisphere with sounds placed symmetrically in the back hemisphere, i.e., points that lay in a cone of confusion. This paper reports on a study that involved the measurement of the HRTFs of 20 subjects, and the analysis of their performance in locating virtual sources in a horizontal plane. The subjects were tested using their own individual HTRFs and also using the HRTFs of a prototype subject who has particularly protruding pinnae. Results indicate that the additional shadowing of sounds from the back hemisphere encoded in the HRTFs of the prototype subject aided some of the test subjects in resolving the front-back confusion observed when tested with their own HRTFs.
2pPP3. Determining the influence of visual stimuli on the peception of surround sound using data mining algorithms. Piotr Odya, Andrzej Czyzewski, Bozena Kostek (Sound and Vision Eng. Dept., Technol. Univ. of Gdansk, 80-952 Gdansk, Poland), and Tomasz Smolinski (Computional Intelligence Lab., Univ. of Louisville, Louisville, KY 40292)

A short description of experiments that aim to determine visual cues influence on the perception of spatial sound is provided in the paper. The earlier stage of the carried out experiments showed that there exists a relationship between the perception of video presented in the screen and sound signals reproduced in a surround system. However, this relationship is dependent on the type of audio-visual signals. Thus a series of subjective tests has been performed on dozens of experts in order to discover these dependencies. The main issue in such experiments is the analysis of the influence of visual cues on the perception of the surround sound. This problem is solved with the application of genetic algorithm and rule searching mechanism to the processing of subjective test results. Some results and conclusions concerning the complexity of the investigated problem are included. [Research sponsored by the Committee for Scientific Research, Warsaw, Poland, Grant No. 8 T11D 00218.]

2pPP4. The "opposite direction shift" phenomenon in sound localization using two sound sources. Hiroyuki Fujii and Kazuhiko Kakehi (CIAIR, Nagoya Univ., Furo-cho, Chikusa-ku, Nagoya-shi 464-8601, Japan)

It has been known that in a situation where tone and noise are presented at once, the perceived direction of the tone is shifted to the opposite direction of the noise. However, it is still not very clear which cues for sound localization are responsible for this "opposite direction shift" phenomenon. In this study, three factors of two sounds were investigated: temporal configuration, frequency overlapping, or level difference of two sounds. The experiment was based on a subjective sound localization task of the designated sound source among two sound sources. The target sound was a $1 \mathrm{kHz}$ or a $500 \mathrm{~Hz}$ tone, and the distractor sound was pink or white noise or, notched noise. A target sound was presented for the cases where the distractor sound was at $25^{\circ}$ left or $25^{\circ}$ right of the loudspeaker array. The perceived direction of the target sound was compared. The "opposite direction shift" phenomenon was observed for the cases where the distractor was longer in duration or had a larger level than the target, or is overlapping with the target frequency. These results suggest that the important perceptual factor of the "opposite direction shift" phenomenon is related to the distractor's "saliency." 
2pPP5. Interaural coherence and the lateralization of noise by interaural level differences. William M. Hartmann and Zachary A. Constan (Michigan State Univ., East Lansing, MI 48824)

Coherent noise, with an interaural cross correlation of unity, and incoherent noise, where signals to left and right ears are independent, sound very different. The former produces a compact lateralized image. The latter produces a diffuse image that fills the head. Nevertheless, listening experiments show that coherent and incoherent noise images seem to be equally affected by small interaural level differences (ILD). The ILD threshold for lateralizing incoherent noise is less than half a dB greater than the threshold for lateralizing coherent noise. In this sense, the human binaural system appears to behave like an ideal level meter, insensitive to the wave form and envelope fine structures that determine coherence. The small discrepancy (less than $0.5 \mathrm{~dB}$ ) can be understood from a standard model of loudness perception-incorporating critical band filtering, halfwave rectification, amplitude compression ( 0.6 power law), and temporal integration $(300 \mathrm{~ms})$. The same model can also account for the small observed difference in lateralization ILD thresholds for coherent noise (NO) and antiphasic noise $(N \pi)$. It can further account for the observed noise bandwidth dependence of lateralization ILD thresholds. [Work supported by the NIDCD.]

2pPP6. Detectability of phase change and its computational modeling. Masato Akagi and Masato Nishizawa (School of Information Sci., Japan Adv. Inst. Sci. \& Tech., 1-1 Asahidai, Tatsunokuchi, Ishikawa 923-1292, Japan, akagi@jaist.ac.jp)

The ability to detect a phase change was measured in paired-stimuli comparison-experiments using monotonic phase (MPH) and phase-shifted $(\mathrm{SPH})$ complex tones. Fundamental frequencies $(F 0)$ were 62.5 to $250 \mathrm{~Hz}$ in 1/4-octave steps. The number of harmonics was set to cover frequencies, 300-9300 Hz. All of the harmonics except one were started in sine phase. The remaining component was shifted pi (SPH) or not shifted (MPH). Subjects indicated whether paired stimuli were "the same or not" and reported whether phase-shifted harmonics appeared to "pop-out." A computational model based on AIM (Patterson, 1995) was constructed and the experimental results were interpreted from autocorrelograms (ACGs) calculated by the model. The findings are as follows. When $F 0$ was below $150 \mathrm{~Hz}$ and the number of harmonics in ERB at the phase-shifted harmonic exceeded 2, a phase change was detected and "pop-out" phenomena occurred. When $F 0$ was over $200 \mathrm{~Hz}$, however, MPH and SPH were not discriminated and there was no "pop out." Discriminable paired stimuli have different ACGs and this reflects the detection ability of phase changes. Fine peaks in the ACG of SPH were found to correspond to the periods of the phase-shifted harmonics. [Work supported by CREST, JST.]

2pPP7. Backward interference of easily identified tones by simple and complex maskers. Richard E. Pastore and Melody S. Berens (Psych. Dept., Binghamton Univ., Binghamton, NY 13902, pastore@binghamton.edu)

There are several different types of interference in the perception of brief target tones due to the presence of subsequent stimulus in close temporal proximity. Backward masking is interference in the detection of the target tone, with the masker usually either a tone or noise. Backward recognition is interference of a tone masker in the discrimination of target tones typically separated by no more than a few jnd's. Components of natural temporal sequences are usually more complex than tones and more structured than noise, with major distinctions contrasted by more than a few jnd's. For example, brief initial position stop consonants are followed by complex vowel formants. The current study examines the ability to differentiate initial tones that, in isolation, are easily detected and distinguished (a musical fifth). In addition to noise, subsequent masking stimuli consisted of all possible combinations of three tones that are easily differentiated from each other (a musical third) and from the target tones. There is little backward interference from noise, small to moderate interference from individual tones, and significant interference from specific combinations of tone maskers. Thus backward interference is not limited to near threshold detection or discrimination.

2pPP8. Pitch interval perception by musically trained listeners. Henning Reetz (Linguist. Dept., Univ. of Konstanz, D186, 78462 Konstanz, Germany, henning.reetz@uni-konstanz.de)

This research investigates the reaction times to identify musical intervals by subjects with some musical training. All intervals within an octave, upward and downward, consisting of all combinations sine tones, complex tones with a fundamental, and complex tones without a fundamental were presented to a group of 18 subjects. Subjects had to decide which signal pairs form a specific musical interval. For some subjects the identification of certain intervals (e.g., a third major upward) was only $20 \mathrm{~ms}$ longer than the identification of two tones with identical pitch but the overall reaction time doubled when the same interval was presented downward. Some subjects showed a great dependency on the presence and absence of the fundamental whereas other subjects were not influenced by this parameter. Furthermore, the sort of musical instrument played by the subjects seem to influence the speed of perception of certain intervals. In total, reaction times indicate that the perception of the missing fundamental is idiosyncratic and depends on the musical education of the subjects and is not necessarily a part of normal auditory processing.

2pPP9. Effect of pitch fluctuation of vowels on vowel perception under a noisy environment. Kentaro Ishizuka and Kiyoaki Aikawa (NTT Commun. Sci. Labs., NTT Corp., Morinosato-Wakamiya 3-1, Atsugi City, Kanagawa 243-0198, Japan)

Previous paper reported that natural vowel identification scores were higher under harmonic complex tones than under a white noise, in spite of the tone and the noise having the same flat spectral envelope [K. Ishizuka and K. Aikawa, J. Acoust. Soc. Am. 109, 2439 (2001)]. Natural vowels have pitch fluctuation. This paper reports a new finding that the pitch fluctuation has the effect of improving vowel identification under noisy environments. An experiment was conducted to examine the effect of pitch fluctuation of vowels on vowel perception under a harmonic complex tone at SNRs ranging from -10 to $-15 \mathrm{~dB}$. The Japanese female vowels were synthesized with the pitches fluctuated according to the $1 / f$ characteristics [N. Aoki and T. Ifukube, J. Acoust. Soc. Am. 106, 423-433 (1999)]. Five pitch fluctuation bandwidth conditions for vowels were tested. The bandwidths ranged from 0 to $4.3 \%(F 0=255.3 \mathrm{~Hz})$. The harmonic complex tone was composed of the same $F 0$ of the vowels and 21 components in the cosine phase. The subjects were forced to choose the vowel they heard for each stimulus. The mean vowel identification scores increased from $33.2 \%$ to $46.5 \%$, proportional to the increase of the pitch fluctuation bandwidth.

2pPP10. A method for the automatic hearing aid fitting employing speech in noise and fuzzy logic. Bozena Kostek (Inst. of Physiol. and Pathol. of Hearing, Pstrowskiego 1, 01-912 Warsaw, Poland) and Andrzej Czyzewski (Sound and Vision Eng. Dept., Tech. Univ. of Gdansk, Narutowicza 11/12, 80-952 Gdansk, Poland)

Some limitations of the hearing aid fitting process are discussed. The classical procedures in this process are based on audiometric test results and/or the loudness scaling method employing artificial test signals. However, the fitting of hearing aids should be also performed on the basis of testing speech understanding in noise, because this is much closer to the real life conditions. A satisfying reliability of these tests may be achieved through the use of modern computer technology with an application of a properly calibrated sound system. A new strategy applicable to fitting prostheses was developed. It allows finding automatically characteristics 
of a hearing aid matching patients needs. The principles of the fitting method employing fuzzy reasoning, and some results of the experiments will be presented in the paper.

2pPP11. Computer simulations of hearing aid acoustical system performance. Grzegorz Szwoch (Sound and Vision Eng. Dept., Tech. Univ. of Gdansk, Narutowicza 11/12, 80-952 Gdansk, Poland) and Bozena Kostek (Inst. of Physiol. and Pathol. of Hearing, Pstrowskiego 1, 01-912 Warsaw, Poland)

The recent developments in the hearing aid technology enabled a number of improvements in hearing aids. This includes advanced signal processing algorithms, better speech intelligibility, miniaturization etc. One of the existing limitations is, however, the problem with providing patientrelated characteristics of the acoustical system of a hearing aid. The aim of this paper is to show that using the physical modeling method it is possible to first build a model of the acoustical system of a hearing aid and then to simulate its performance. The waveguide model of the acoustical system of a hearing aid is proposed. Exemplary results of the computer simulations using such a model are presented and compared with some measurement data of existing hearing aid acoustical systems. The model proved to behave similarly to the real system. Conclusions regarding the application of such a method in the fitting process of a hearing aid are included.

2pPP12. Adaptive dual-microphone directional noise reduction scheme for hearing aids using nearby microphones. Martin Kompis and Matthias Bettler (Univ. Clinic of ENT, Head and Neck Surgery, Inselspital, 3010 Berne, Switzerland)

A new directional dual-microphone noise reduction scheme for hearing aids is presented. The acoustic field is sampled by two nearby microphones, which can be mounted conveniently in a single behind-the-ear or in-the-ear unit, such as a hearing aid or the external part of a cochlear implant system. A combination of time-invariant and adaptive postprocessing of the microphone signals is employed. First, time-invariant signal processing is used to combine the two microphone signals into two intermediate signals, one containing predominately sounds arriving from the front, one predominately from the rear. These signals are postprocessed by an adaptive noise canceller using filter lengths between 1 and $50 \mathrm{~ms}$. Experiments have been performed in several simulated, realistic acoustic environment. In a moderately reverberant room (reverberation time $0.4 \mathrm{~s}$, volume $34 \mathrm{~m}^{3}$ ) improvements in signal to noise ratio (SNR) of 3.4-8.7 $\mathrm{dB}$ were achieved, depending on the length of the adaptive filter and the angle of incidence of the noise signal. A classic time-invariant dualmicrophone approach yielded $0.2-6.9 \mathrm{~dB}$ lower SNRs in the same acoustic settings. The computational load of the proposed system is well within reach for current hearing aid technology. [Work supported by the Swiss National Science Foundation.]

2pPP13. Model studies of source term and reflection components of synchronous-evoked and distortion product otoacoustic emissions. Carrick Talmadge (Univ. of Mississippi, Natl. Ctr. for Physical Acoust., Oxford, MS 38677) and Arnold Tubis (Univ. of California, San Diego, La Jolla, CA 92093)

The unified model of synchronous-evoked, distortion product, and spontaneous otoacoustic emissions of Talmadge et al. [J. Acoust. Soc. Am. 104, 1517 (1998); 105, 275 (1999); 108, 2570 (2000)] provides a useful testing ground for various paradigms that have been proposed for separately determining source terms and reflectance components of synchronous-evoked and distortion-product otoacoustic emissions. The source terms are the ear canal components that would exist in the absence of effects associated with cochlear wave reflections due to, e.g., distributed inhomogeneities [Zweig and Shera, J. Acoust. Soc. Am. 98, 2018 (1995)] and nonlinearity [Talmadge et al., 2000]. Model calculations are used to compare results for the unmixing of these components obtained using reflection-suppression techniques [see, e.g., Kalluri and Shera, J. Acoust. Soc. Am. 109, 622 (2001)] and various forms of time-domain latency windowing ones [see, e.g., Knight and Kemp, J. Acoust. Soc. Am. 107, 457 (2000)]. Because of multiple internal wave reflections at the cochlear base and the tonotopic place for the cochlear waves, the model implies (and experimental data indicate) that the reflectance components will generally exhibit fine structure effects similar to those of the total ear canal signal.

2pPP14. High-frequency stimulation in tinnitus treatment. Martin L. Lenhardt, Douglas G. Richards, Alan G. Madsen (Prog. in Biomed. Eng., Virginia Commonwealth Univ., Box 980158 MCV, Richmond, VA 23298-0168), Abraham Shulman, Barbara A. Goldstein, and Robert Guinta (Martha Entenmann Tinnitus Res. Ctr., Brooklyn, NY 11203)

Limited but convincing evidence suggests neural plasticity plays a role in the development and maintenance of tinnitus. Tinnitus is generally pitch matched with high frequencies $(<6 \mathrm{kHz})$ and is often associated with very hearing loss, although the conventional audiometric thresholds may be normal or near normal. It is hypothesized that the brain experiences high-frequency air conduction auditory deprivation and this is a causative factor in tinnitus. If the low-pass middle ear is circumvented by bone conduction stimulation, residual high-frequency receptors in the cochlea can again be activated. High-frequency bone conduction transducers were fabricated to deliver frequencies from 6 to $40 \mathrm{kHz}$. The transducers were aluminum ceramic bimorphs with circular topology which were heavily damped by mass loading to the mastoid with resonances at 9 and $39 \mathrm{kHz}$. Ten subjects with persistent tinnitus were selected to listen to proprietary high-frequency patterned stimulation for two half-hour sessions for 4 weeks. Exit questionnaires revealed satisfaction with tinnitus relief in the majority $(60 \%)$ with residual inhibition lasting on the order of weeks (mean $=1.5$ weeks). Encouraged by these findings, more extensive trials are underway to explore the efficacy of high-frequency bone conduction treatment in central tinnitus.

2pPP15. Digital USB audiometer with noise-shaping techniques for signal generation. Antonio Minguez, Juan Sancho, Jorge Grundman, and Vladimir Ulin (EUIT Telecomunicacion, Ctra. Valencia km. 7, 28031 Madrid, Spain)

A digital audiometer has been implemented on a personal computer with new features to make a low cost and simple equipment. The signals are generated digitally (digital samples) by a recursive algorithm that shapes the spectrum of the quantization noise of the digital samples in order to: (1) eliminate the harmonic distortion at low levels and (2) to increase the signal-to-noise ratio of every tone generated, as the standard EN 60645-1 requires for tone audiometers. Besides, the digital output of the personal computer (PC), the universal serial bus (USB), is used to link the PC with a compatible USB headphones so the transducer can get the original signal in a digital format, avoiding all the analog and electrical noise and distortion in the PC environment, so the proposed audiometer does not need any external equipment to generate the test signals, reducing the total cost of the product.

2pPP16. Implementation of narrow-band noise algorithms according to ANSI S3.6-1996. Mariano Ruiz, Ruben Salvador, and Manuel Recuero (Universidad Politecnica de Madrid, INSIA Crta. Valencia Km-7, Madrid 28031, Spain)

In order to generate narrow-band noise according to ANSI S3.6-1996 in an efficient way, it is necessary to employ multirate signal processing techniques. Thanks to the successive conversion of the sampling rate in the stages of the system, an efficient implementation of the required algorithms is obtained. These signals are entirely bandpass signals. The adopted solution is to design equivalent lowpass filters followed by a frequency modulation of the filtered signal with the purpose of moving it 
to the desired frequency. To obtain a better performance in the resulting filter, multistage structures have been used to achieve the required changes in the sampling rate to implement the lowpass filter by means of a multirate signal processing technique. A LabVIEW application have been implemented for designing these types of filters. This program generates the filter coefficients and offers the possibility of carrying out a simulation and saving these coefficients into a $\mathrm{C}$ header file. These filters have been successfully tested, on a new DSP based audiometer that uses TI TMS320C6701, by means of Code Composer Studio and a series of routines that implement each one of the stages that compose the filter.

\title{
Session 2pSA
}

\section{Structural Acoustics and Vibration: Methods of Passive Dissipation in Structural Acoustics II}

\author{
Courtney B. Burroughs, Chair \\ Applied Research Laboratory, Pennsylvania State University, P.O. Box 30, State College, Pennsylvania 16804
}

\section{Invited Papers}

\begin{abstract}
2:00
2pSA1. Structural damping as a result of piezoelectric energy harvesting. George A. Lesieutre (Aerosp. Eng., Penn State Univ., University Park, PA 16802), Heath Hofmann, and Geffrey Ottman (Elec. Eng., Penn State Univ.)

This paper describes an approach to harvesting electrical energy from a mechanically-excited piezoelectric element. If the piezoelectric element is attached to a vibrating structure, this energy transfer also results in structural damping. The energy harvesting approach is loosely based on the fundamental electrical engineering concept of optimal loading. The harvesting circuit consists of an ac-dc rectifier with an output capacitor, an electrochemical battery, and a switch-mode dc-dc converter that controls the energy flow into the battery. An adaptive control technique was initially used to vary the switching duty cycle so as to maximize power transfer into the battery. Experiments demonstrated that the adaptive dc-dc converter increased power transfer by over $400 \%$, as compared to direct connection of the rectifier output to the battery. For persistent excitation above a certain level, the existence of a near-optimum duty cycle was also observed. A standalone energy harvesting system that exploited this observation was developed. This system has two modes: for low excitation, the rectifier charges the battery directly; while for higher excitation, the battery runs a fixed duty cycle $\mathrm{dc}-\mathrm{dc}$ converter. This standalone system was successfully demonstrated in the laboratory. Effective damping loss factors associated with this energy harvesting scheme are also reported.
\end{abstract}

\section{$2: 30$}

2pSA2. Real-time switching of piezoelectric shunts for structural vibration control. William W. Clark (648 Benedum Hall, Mech. Eng. Dept., Univ. of Pittsburgh, Pittsburgh, PA 15261)

The use of piezoelectric actuators to passively add damping to vibrating structures has been investigated for over a decade. Simple resonant shunt circuits consisting of resistors and inductors are connected to the piezoelectric actuators to add damping to one of the structural modes [Hagood and Crawley, J. Guid. Control Dyn. 14]. Modifications to the method have been proposed to enable a multi-modal damping technique, but at the cost of performance to any one mode [e.g., Wu, Proc. of 1998 SPIE Smart Structures and Materials Conference, Vol. 3327, pp. 159-168]. The objective of this talk is to discuss recent research into methods that help eliminate the disadvantages of passive resonant shunt damping. The methods to be discussed involve real-time switching of piezoelectric shunt circuits, and are based on the foundations laid by Larson [Ph.D. Dissertation, Georgia Institute of Technology, 1996], Clark [J. Intell. Mater. Syst. Struct. 11, 263-271], and Richard et al. [Proc. of 1999 SPIE Smart Structures and Materials Conference, Vol. 3672, pp. 104-111]. This talk will begin with the basic concepts of real-time switching, and will lead up to recent research that has demonstrated the use of the technique for multi-modal vibration suppression.

\section{3:00}

2pSA3. A nonlinear semipassive vibration damping using piezoelectric elements. Daniel J. Guyomar, Claude L. Richard, and David S. Audigier (INSA, LGEF, Bt 504, 8 Rue de la Physique, 69621 Villeurbanne, France)

The proposed approach is based on a nonlinear filtering of the piezoelements output voltage and does not require any tuning to ensure damping performances over a large bandwidth. The output voltage is short circuited for a very brief period of time when the voltage reaches a maximum or a minimum. As a consequence of this filtering, the voltage gets distorted, amplified, and shifted in the time domain. It can be split in two signals, one is in phase with the vibration displacement and contributes to the potential energy while the second is in phase with the vibration speed and participates to the energy damping. The technique is semipassive since it requires a very low electrical power to drive the switch sequences. Experimental data show that the switching technique leads to better damping performances than purely passive methods whatever the natural damping of the structural material is. Furthermore, switching the piezovoltage through a small inductor leads to a voltage amplification and consequently to an increase of the damping. Damping capabilities range from $6 \mathrm{~dB}$ for an epoxy beam to nearly $20 \mathrm{~dB}$ for a steel beam even in the low frequency regime. Experimental results and theoretical interpretations will be presented. 
2pSA4. Absorption and trapping of vibration energy by complex resonators. Antonio Carcaterra (INSEAN, Istituto Nazionale per Studi ed Esperienze di Architettura Navale, Via di Vallerano, 139, 0128, Rome, Italy) and Adnan Akay (Mech. Eng. Dept., Carnegie Mellon Univ., Pittsburgh, PA 15143)

This presentation examines conditions that can enhance apparent damping of a master structure due to its coupling with a complex resonator that does not exhibit classical dissipation properties. The model uses a set of multistage parallel oscillators within which energy is trapped. The method presented here for predicting the apparent damping is based on evaluation of the asymptotic response of the master structure utilizing the Hilbert transform. The result is a functional relationship between the frequency distribution within the system and the resulting apparent damping. This study leads to a nonclassical dynamic absorber, consisting of a multistage parallel oscillators, in terms of its absorption and trapping characteristics. The energy is absorbed from the master at a rate determined by the apparent damping and trapped for a given time interval as measured by the return time. The theoretical results suggest optimal configurations to decrease the absorption time and to increase the trapping time (up to an infinite delay). The results of numerical simulations performed on complex vibro-acoustic systems support the proposed theoretical approach. [Research sponsored by INSEAN and NSF.]

\section{4:00-4:15 Break}

\section{Contributed Papers}

\section{4:15}

2pSA5. Use of the two channels method to determine the guided waves damping coefficients of an asymmetrically fluid-loaded plate: Theory and experiment. Ech-Cherif El-Kettani Mounsif and Franklin Herve (Laboratoire d'Acoustique Ultrasonore et d'Electronique, L.A.U.E. U.M.R. C.N.R.S. 6068, Universit du Havre, Pl. Robert Schuman, 76610 Le Havre, France)

Exact and approximate formalisms describing the interactions of acoustic plane waves with an elastic isotropic plate immersed between two different fluids are presented. This is an extention of the Fiorito, Madigosky, and Berall (FMU) theory. Resonant approximations are given for the reflection and transmission coefficients, which assume light fluids loading. These approximations show that the resonance widths are the sum of two independent partial widths, each of them being related to one fluid and to the plate physical properties. In addition, experimental results are presented for a steel plate; the damping coefficients of the propagating leaky Lamb waves along the loaded plate are measured in two different loading situations. In the first situation the plate is embedded in water (water loading on both sides), while in the second situation it is loaded with water on one side and acetone on the other side. The experimental damping coefficient of the acetone-steel-acetone structure is deduced therefrom. The damping coefficient obtained in each case is then compared to the theoretical half resonance width, and the experimental results are found to be in good agreement with the theoretical predictions.

\section{$4: 30$}

2pSA6. Analytical solutions for characterizing tortuosity and characteristic lengths of porous materials using acoustical measurements: Indirect model. Xavier Olny, Jerome Tran Van (Ecole Nationale des Travaux Publics de l'Etat, DGCB URA CNRS 1652, rue Maurice Audin, 69518 Vaulx en Velin, France), and Raymond Panneton (GAUS, Dept. of Mech. Eng., Univ. de Sherbrooke, QC J1K 2R1, Canada)

Under the homogenization hypothesis, wave propagation in porous materials can be described using, for example, macroscopic dynamic density and bulk modulus. These functions depend on statistical geometrical parameters. Classically, five parameters are used: porosity, static air flow resistance, tortuosity, and viscous and thermal characteristic lengths. The three last parameters are generally difficult to measure with existing direct methods, for a wide range of materials. The proposed method is based on the measurement of dynamic density and compressibility, in order to separate viscous and thermal effects. With prior knowledge of airflow resistance and porosity, it is then possible to find analytical solutions for the missing parameters, fitting Johnson-Allard-Champoux model. In the same way, we also tackle the problem of the determination of static "thermal permeability" introduced to improve the description of thermal dissipation effect. Experimental results, obtained with various materials, using a Kundt's tube, are presented to show the efficiency, and relative simplicity of the method. Moreover, the relevance of using a three parameters model for describing the bulk modulus is discussed.

\section{$4: 45$}

2pSA7. Analytical solutions for characterizing tortuosity and characteristic lengths of porous materials using acoustical measurements: Extrapolation model. Raymond Panneton (GAUS, Dept. of Mech. Eng., Univ. de Sherbrooke, QC J1K 2R1, Canada), Xavier Olny, and Jerome Tran Van (Ecole Nationale des Travaux Publics de l'Etat, DGCB URA CNRS 1652, rue Maurice Audin, 69518 Vaulx en Velin, France)

Following sophisticated descriptive models on sound propagation in porous media, five acoustical parameters usually describe the complexity of the porous network: porosity, static air flow resistance, tortuosity, and viscous and thermal characteristic lengths. While porosity and airflow resistance can be measured directly and accurately using simple apparatuses, simple and accurate direct measurements of the tortuosity and characteristic lengths are still an open research area. Actual researches on the characterization of these parameters use acoustical methods in the ultrasound frequency range. In this paper, a similar approach is investigated, but applying this time in the frequency range $2000-6000 \mathrm{~Hz}$. The method is based on analytical solutions linking the tortuosity and characteristic lengths to measured dynamic density and compressibility. Following an extrapolation procedure to infinite frequency, the tortuosity and characteristic lengths are deduced without prior knowledge of porosity and air flow resistance. Experimental results from Kundt's tube are compared to simulations on various materials to show the accuracy and limitations of the method. 
Session 2pSC

\title{
Speech Communication: Cross-Language Issues (Poster Session)
}

\author{
Megha Sundara, Chair \\ School of Communication Sciences and Disorders, McGill University, 1266 Pine Avenue, West, \\ Montreal, Quebec H3Q 1A8, Canada
}

\section{Contributed Papers}

\begin{abstract}
All posters will be on display from 1:30 p.m. to 5:00 p.m. To allow contributors an opportunity to see other posters, contributors of odd-numbered papers will be at their posters from 1:30 p.m. to 3:15 p.m. and contributors of even-numbered papers will be at their posters from 3:15 p.m. to 5:00 p.m.
\end{abstract}

2pSC1. On talker voice in language identification. Verna Stockmal and Z. S. Bond (Dept. of Linguist., Ohio Univ., Athens, OH 45701)

Listeners find that discriminating between two languages is relatively easy compared to identifying new samples. Listener responses seem to be influenced by regional speech characteristics and talker voice quality. This study attempted to assess listener ability to match spoken samples of unknown languages when produced by male and female talkers. Samples from Arabic and Latvian, three provided by male talkers and three by female talkers, were assembled in a test using $\mathrm{ABX}$ format. Listeners matched the $\mathrm{X}$ language with either the A or B language, across same sex and different sex talkers. Overall, listeners performed at above chance levels, $60 \%$ correct. Listeners identified the two languages at approximately equal rates. They matched speech samples produced by males $(75 \%)$ better than speech samples produced by females (53\%). Listeners matched languages across speaker gender at 55\% correct. These results suggest that speaker sex, as part of voice quality, is encoded with a representation of the unknown language.

2pSC2. Non-native speech production I: Listener-talker adaptation. Ann R. Bradlow and Tessa Bent (Dept. of Linguist., Northwestern Univ., 2016 Sheridan Rd., Evanston, IL 60208)

To assess native listener adaptation to foreign-accented speech, we submitted 16 sentences produced by three talkers of Chinese-accented English to intelligibility testing. First, the test sentences were orthographically transcribed by native English listeners in a single-talker format in which the test sentences from one talker were preceded by 32 , and followed by 16 , other sentences produced by the same talker. Second, the test sentences were presented to native listeners in a multiple-talker format in which the test sentences from one talker were interspersed with 48 similar sentences produced by 31 other non-native talkers from various language backgrounds. Within the single-talker condition, the native listeners' transcription accuracies for all three talkers improved from the beginning to the end of the test session. The magnitude of this improvement was similar across all three talkers. Furthermore, for all three talkers, intelligibility was better in the single-talker condition than in the multiple-talker condition; however, this difference depended on the talker's overall intelligibility. The "best" and "worst" talkers showed smaller single- versus multiple-talker differences than the "medium" talker. These findings demonstrate that foreign-accented speech intelligibility can improve as the listener gains experience with the voice and speech patterns of the individual non-native talker. [Work supported by NIH-NIDCD Grant No. DC03176.]
2pSC3. Non-native speech production (II): phonemic errors by position-in-word and intelligibility. Tessa Bent (Dept. of Linguist., Northwestern Univ., 2016 Sheridan Rd., Evanston, IL 60208, t-bent@northwestern.edu), Bruce L. Smith, Danielle Lodewyck (Dept. of Commun. Sci. and Disord., 2299 N. Campus Dr.), and Ann R. Bradlow (Dept. of Linguist.)

This study considered the distribution of non-native talker phonemic errors by position-in-word, and their differential effects on non-native speech intelligibility. Based on the structure of words in the native language of the talkers (i.e., Chinese), it was hypothesized that word-initial phonemes would be produced more accurately than word-final phonemes. Additionally, based on models of lexical access (e.g., cohort model), it was hypothesized that phonemic errors in the word-initial position would be more detrimental to intelligibility than phonemic errors later in the word. 15 talkers of Chinese-accented English produced 16 simple declarative sentences. These recordings were phonetically transcribed to determine the number and type of production errors and presented to native English listeners for intelligibility testing. Results showed that, as predicted, wordinitial phonemes were produced more accurately ( $92 \%$ correct) than wordfinal phonemes (82\% correct) $[t(14)=3.815, p<0.01]$. Furthermore, word-initial singleton consonants and vowels correlated with intelligibility (Rho $=0.738, p<0.01$ and Rho $=0.692, p<0.01$, respectively). In contrast, initial and final consonant clusters, word-final singleton consonants, and within-word consonants were not significantly correlated with intelligibility. These results demonstrate that, for a variety of talker- and listenerrelated reasons, a phoneme's position within the word exerts an important influence on both its production and perception. [Work supported by NIHNIDCD Grant No. DC03176.]

2pSC4. The pronunciation of English sentences by Korean children and adults. J. E. Flege (Div. of Speech \& Hearing Sci., Univ. of Alabama at Birmingham, CH20, 1530 3rd Ave. S., Birmingham, AL 35294, jeflege@uab.edu), David Birdsong (Univ. of Texas, Austin, TX 78712), Ellen Bialystok (York Univ., Tononto, ON M3J 1P3, Canada), Molly Mack (Univ. of Illinois, Urbana, IL 61801), and Hyekyung Sung (Stanford Univ., Stanford, CA 94305)

This study examined English sentences produced by four groups of native Korean subjects (18 each) who differed according to mean age (children $=12$ years, adults $=32$ years) and length of residence in North America (means $=3$ vs 5 years). A delayed repetition technique was used to elicit English sentences at Time 1 and one year later at Time 2. Native English-speaking listeners used a 9-point scale to rate the sentences for overall degree of foreign accent. The ratings obtained for the native Korean $(\mathrm{NK})$ subjects were converted to $z$-scores using the mean ratings and standard deviations obtained for sentences produced by control groups of Native children and adults. As expected, analyses of the standardized rat- 
ings revealed that the NK children produced the sentences with milder foreign accents than the NK adults did at both Time 1 and Time 2. Unexpectedly, the adult-child difference was larger at Time 2 than Time 1 because the NK children's foreign accents diminished whereas the NK adults' foreign accents grew significantly stronger from Time 1 to Time 2 . Possible explanations for this are age-related differences in motivation, English input, or the strength of influence of Korean phonetic structures on the English sound system.

2pSC5. Intonation patterns in productions by Seoul Korean speakers of English. Julia Tevis McGory (Ohio State Univ., Dept. of Speech \& Hearing, 110 Pressey Hall, 1070 Carmack Rd., Columbus, OH 43210)

This study compares the intonation patterns produced in English declaratives and yes-no questions by native American English speakers and native Korean speakers. As is well known, stress and pitch accent are integral to English intonation. Declaratives are produced with an $F 0$-rise $\left(\mathrm{L}+\mathrm{H}^{*}\right)$ in the stressed syllable of the most prominent word followed by a steep utterance-final fall while yes-no questions are produced with a sharp $F 0$-fall ( $\left.\mathrm{L}^{*}\right)$ onto the stressed syllable followed by a gradual utterancefinal rise. Words that follow the focused word are not associated with any particular $F 0$ pattern. In contrast, Korean has neither stress nor pitch accent. Prominent words in statements and questions are produced at the beginning of a phrase and realized with an $F 0$-rise $(\mathrm{LH})$ followed by a gradual fall to the end of statements $(\mathrm{L} \%)$ and a final rise $(\mathrm{LH} \%)$ in questions. In this study, subjects from both subject groups produced the words "memorial" and "memorizes" in both focus and post-focus positions in both statements and yes-no questions. The question of interest is whether Korean speakers follow the intonational pattern of English (their L2) or utilize the intonational characteristics of their native language.

2pSC6. Bilingual speech production: The case of simultaneous acquisition. Megha Sundara and Linda Polka (School of Commun. Sci. and Disord., McGill Univ., 1266 Pine Ave. W., Montreal, QC H3G 1A8, Canada)

Despite increased interest in people learning a second language (L2) in the past decade or two, there have been few studies investigating production by bilinguals at the segmental level. In this experiment coronal stop consonants in real-word productions in French and English by bilingual French-English speakers, very fluent in both languages having learned the two languages simultaneously since birth, were recorded. Differences in the phonetics of Canadian French and English include VOT and place of articulation distinctions. Canadian French has a two-way voicing distinction between prevoiced and simultaneous release stops and uses the dental place of articulation. Canadian English, on the other hand, has a two-way voicing distinction (in syllable initial position) between simultaneous and aspirated release for stops and uses an alveolar place of articulation. Acoustic analyses of stop consonants for both VOT and place of articulation (dental versus alveolar) are reported. Productions by bilinguals will be compared with those of respective monolingual groups for similarities and differences. Results will be discussed with respect to Grosjean's wholisticfractionated classification of the bilingual system [F. Grosjean, Brain and Language 36, 3-15 (1989)].

2pSC7. Articulatory gestures of /// by bilingual Korean-English children. Sunyoung Oh (Dept. of Linguist., Univ. of British Columbia, E270-1866 Main Mall, Vancouver, BC V6T 1Z1, Canada)

Properties of articulatory gestures (e.g., timing and magnitude) have been used to find the phonetic correlates of syllable position. For example, allophones of English /1/ are determined by the timing relationship between the tongue tip and dorsum gestures, where greater timing lag is observed in the post-vocalic position. On the other hand, allophones of Korean /1/ are determined by the position of the tongue tip, prevocalic post-alveolar versus post-vocalic alveolar. The magnitude of both gestures is also greater in post-vocalic position, and the tongue dorsum is slightly advanced in Korean. This paper examines cross-language influences of the articulatory gestures of $/ 1 /$. To test this, an ultrasound study of the speech of two bilingual Korean-English children was conducted. The results showed that bilingual children produced /1/ using the correct tongue tip gestures of Korean in both positions (post-alveolar versus alveolar); however, they failed to implement tongue dorsum advancement. Instead, they incorrectly adopted the English-like gesture and implemented tongue dorsum retraction in post-vocalic positions. These indicate that bilingual children are attuned to language-specific gestures of both languages and there is at least a one-way effect (English to Korean) on /1/ gestures.

2pSC8. Perception and production of English consonants by Japanese adults and children. Katsura Aoyama (Div. of Speech \& Hearing Sci., Univ. of Alabama at Birmingham, CH20, 1530 3rd Ave. S., Birmingham, AL 35294, aoyama@uab.edu), Susan Guion (Univ. of Oregon, Eugene, OR 97403), J. E. Flege (Univ. of Alabama at Birmingham, Birmingham, AL 35294), Tsuneo Yamada (National Inst. of Multimedia Education, Chiba 261-0014, Japan), and Reiko Akahane-Yamada (ATR Human Information Processing Res. Labs., Kyoto 619-0288, Japan)

This study examined perception and production of English consonants by native Japanese (NJ) adults and children (16 subjects in each group, mean age $=39.9$ and 9.9 years). The subjects were tested 2 times $(\mathrm{T} 1, \mathrm{~T} 2)$, when their mean lengths of residence in the U.S. were 0.5 years and 1.6 years. Age-matched native English (NE) controls (16 adults, 16 children) also participated. A categorial discrimination task was used to assess the perception of the English contrasts $/ \mathrm{b} /-/ \mathrm{s} /, \mathrm{b} /-/ \mathrm{v} /, / \mathrm{I} /-/ 1 /, / \mathrm{I} /-/ \mathrm{w} /$, and $/ \mathrm{s} /-/ \theta /$. For three groups (NE adults, NE children, NJ adults), the T1 and T2 scores did not differ significantly on any contrast. The NJ children's scores were significantly higher at $\mathrm{T} 2$ than at $\mathrm{T} 1$ on the $/ \mathrm{I} /-/ \mathrm{l} /$ and $/ \mathrm{I} /-/ \mathrm{W} /$ contrasts. A picture naming task with auditory cues were used to elicit the production of English words containing consonants of interest. Twelve NE listeners identified the subjects' production of $/ \mathrm{I} /, / \mathrm{l} /$, and $/ \mathrm{w} /$ in wordinitial position. Only the NJ children's scores improved significantly from $\mathrm{T} 1$ to $\mathrm{T} 2$, and their improvement was the greatest on the production of $/ \mathrm{I} /$. Several possible explanations will be offered for why there seems to be more improvement from $\mathrm{T} 1$ to $\mathrm{T} 2$ for $/ \mathrm{I} /$ than $/ \mathrm{l} /$.

2pSC9. Acoustic analysis of American listeners' Mandarin tone productions before and after perceptual training. Yue Wang (Ctr. for Mind, Brain, and Learning, Box 357988, Univ. of Washington, Seattle, WA 98195), Allard Jongman, and Joan A. Sereno (Linguist. Dept., Blake Hall, Univ. of Kansas, Lawrence, KS 66045)

Training American listeners to perceive Mandarin tones has been shown to be effective, with the trainees' identification improving by $21 \%$ [Y. Wang, A. Jongman, and J. Sereno, J. Acoust. Soc. Am. 106, 36493658 (1999)]. The effect on production as a result of perceptual training was first assessed by native Mandarin listeners' evaluation of the trainees' tone productions, showing that post-training productions were $18 \%$ more accurately identified than pre-training productions [Y. Wang, M. Spence, A. Jongman, and J. Sereno, J. Acoust. Soc. Am. 105, 1095 (1999)]. A detailed acoustic analysis has now been performed to further examine the nature of the production improvement. Trainees' productions include 80 Mandarin words recorded both before and after perceptual training. For comparison, these words were also produced by native Mandarin speakers. For each production, a pitch track was derived. Results show that posttraining tone contours approximate native norms to a greater degree than pre-training tone contours, indicating an improvement in production following perceptual training. Furthermore, pitch height and pitch contour are not mastered in parallel, with the former being more resistant to improvement than the latter. The results are discussed in terms of the production-perception relationship and native language influence. 
2pSC10. Discrimination of Thai tones by naive and experienced native English speakers. Ratree P. Wayland and Susan G. Guion (Dept. of Linguist., 4131 Turlington, Univ. of Florida, Gainesville, FL 32611-5454)

This study investigated the ability to discriminate between the low and the mid tones of Thai by two groups of native English speakers: naive and experienced. The naive group was comprised of eight native American English speakers who had no previous exposure to Thai or any tonal language. The eight native English speakers in the experienced group had been learning and had lived in Thailand for a varying amount of time. Eight native speakers of Thai participated as control subjects. Stimuli were eight minimal pairs. Three variables were manipulated: (1) experience with the target language: naive vs experienced; (2) syllable types: closed vs open; and (3) levels of processing: phonetic vs phonological implemented by using two different ISI (500 ms and $1500 \mathrm{~ms})$. It was found that experience with the target language resulted in improved discrimination of the target language's tonal contrast. Moreover, all three groups of subjects performed better with closed syllables than open syllables. Contrary to previous research, the effect of ISI was found only for the most difficult contrasts and only for the experienced group. An explanation for the findings and implication for futher research will be offered and discussed.

2pSC11. Perception of English vowels by native Korean and Japanese speakers. Takeshi Nozawa (Kansai Univ. of International Studies, 1-18 Aoyama Miki, Hyogo 673-0521, Japan) and Elaina M. Frieda (Dept. of Speech and Hearing Sci., Ohio State Univ., 110 Pressey Hall, 1070 Carmack Rd., Columbus, OH 43210-1002)

Native speakers of Korean and Japanese were assessed in their ability to perceive English vowels categorically. These two languages were chosen because they differ in the size of their vowel inventories with Korean containing more vowels than Japanese. The Korean vowel space is presumably partitioned more so than the Japanese and this may facilitate Korean speakers' discrimination of English. This hypothesis is in keeping with Best's Perceptual Assimilation Model (PAM). PAM states that nonnative speakers' discrimination of non-native sounds can be predicted by differing degrees based on whether the new non-native sounds are assimilated to one or more native phonemic categories. Further, discrimination is mediated by how well the non-native category fits the native category. Both subject groups completed the following tasks: the Categorical Discrimination Test (CDT, designed by J. Flege) which tested their discrimination of English vowels; and an assimilation task where subjects equated English vowels to their native vowels and rated each for category goodness. A' scores were calculated for each contrast in the CDT. In addition, confusion matrices were calculated for each group. For both subject groups, the English vowels assimilated to a single vowel category were found to be more difficult to discriminate.

2pSC12. Perception of coarticulation with Japanese voiced and voiceless vowels. Tomomi Hashimoto (Prog. in Linguist., Univ. of Florida, 4131 Turlington Hall, Gainesville, FL 32611, tomomi@ufl.edu)

Two experiments investigate whether coarticulatory information of voiced and devoiced vowels in their preceding segments can be exploited by speakers of different dialects and/or by second language learners. In standard Tokyo Japanese, the high vowels $[\mathrm{i}, \mathrm{u}]$ are devoiced between voiceless segments while vowel devoicing is not typical in Osaka dialect. This study examines how vowel's voicing status affects listeners' ability to exploit coarticulatory information in initial consonants. In both experiments, listeners consisted of native speakers of Tokyo and Osaka dialects and native speakers of English learning Japanese as a second language. In the first experiment, the post-alveolar fricative /(esh)/ followed by a vowel, either /i/ or /u/, was produced in real words by native speakers of Tokyo and Osaka dialects. The listeners were presented with a series of excised /(esh)/ to determine the original vowel identity. The second experiment replicated the first experiment testing with the velar stop $/ \mathrm{k} /$. The preliminary results suggest vowel devoicing may cause perceptual diffi- culties in the fricative-vowel context, but not in the stop-vowel context. The results also indicate a difference in cue weighting among 3 groups. Explanation for this finding will also be explored by way of acoustic measurements.

2pSC13. Characteristics and intelligibility of Japanese-accented English. Kanae Nishi (Univ. of South Florida, 4202 E. Flower Ave., Tampa, FL 33620)

The relationship between intelligibility and the degree of accentedness of Japanese-accented English was studied using an accentedness rating task, an identification task, and multidimensional scaling (MDS). Native speakers of American English (AE) listened to speech samples of $270 \mathrm{AE}$ monosyllable words recorded by three groups of speakers (native $\mathrm{AE}$, Japanese residing in the United States, and Japanese who had never lived outside Japan). The length of stay in the U.S. was positively related to native-like dimensionality in MDS solutions and the correct identification of words, but unrelated to ratings of foreign accentedness. High intelligibility was not necessarily achieved by less-accented speakers but by speakers with well-differentiated dimensional structures.

2pSC14. Native and non-native perception of phonemic length contrasts in Japanese. Hiroaki Kato, Keiichi Tajima, and Reiko Akahane-Yamada (Information Sci. Div., ATR Inst. Internal, 2-2-2, Hikaridai, Seika-cho, Soraku-gun, Kyoto 619-0288, Japan, kato@isd.atr.co.jp)

Segment length is distinctive in Japanese, e.g., /kado/ "corner" versus /ka:do/ "card," unlike languages such as English. To study this property extensively and precisely, a series of perception experiments were conducted. Stimuli were pairs of unaccented 3- or 4-mora nonsense Japanese words of the basic form /ere_e/ where _ is one of / $\mathrm{p} \mathrm{t} \mathrm{k} \mathrm{m} \mathrm{n} \mathrm{s/.} \mathrm{Each} \mathrm{pair}$ contrasted in either vowel length, e.g., /erete/ versus /ere:te/, or in consonant length, e.g., /erete/ versus /erette/. Each pair was also embedded within a carrier sentence. A synthetic continuum gradually varying in the duration of the target vowel or consonant was built for each pair using STRAIGHT, a high-fidelity speech analysis, synthesis, and manipulation system [Kawahara et al., Speech Commun. 27, 187-207 (1999)]. Native Japanese and native English speakers participated in both identification and discrimination tests of each continuum. Preliminary results from stimuli containing stops indicate that the non-native discrimination scores were significantly poorer than the native scores, particularly when the continua were embedded in a sentence. The non-native identification scores, however, were similar to those of the native. Implications of these findings are discussed along with results from the fricative and nasal continua.

2pSC15. Cross-dialect perception of English vowels by native Japanese speakers. Elaina M. Frieda and Robert A. Fox (Speech Percept. and Acoust. Lab, Speech and Hearing Sci., Ohio State Univ., 110 Pressey Hall, 1070 Carmack Rd., Columbus, OH 43210-1002, frieda.1@osu.edu)

The present study will examine the influence of dialect on second language acquisition in terms of perceptual abilities. Our lab is currently comparing vowel productions from native Japanese (NJ) speakers with native American English speakers residing in two divergent dialectal regions of the United States (Alabama and Ohio). This study is an elaboration of this work that explores the extent to which there is regional variation in the perceptual responses in $\mathrm{NJ}$ as a function of local dialect. We recorded sets of words from four groups of subjects: NJ subjects residing in Alabama (JA) and Ohio (JO) and native English (NE) subjects from Alabama (EA) and Ohio (EO). These tokens were then used in an identification experiment utilizing the same four subject groups. The experiment addresses at least two different questions: (1) Does the intelligibility of Japanese vowels change as a function of dialect for the English listeners? 
(2) Does the perceptual responses of the Japanese listeners to the EA and EO tokens vary as a function of their ambient linguistic context? The results will be discussed in terms of second language acquisition and dialect acquisition. [Work supported, in part, by an INRS Award from the NIH to Fox, P.I.]

2pSC16. Brain plasticity in behavioral and neuromagnetic measures: A perceptual training study. Yang Zhang, Patricia K. Kuhl (Ctr. for Mind, Brain, and Learning \& Dept. of Speech and Hearing Sci., Univ. of Washington, Seattle, WA 98195), Toshiaki Imada (Nippon Telegraph and Telephone Corp., Atsugi, Kanagawa 243-0198, Japan), Makoto Kotani (Tokyo Denki Univ., Chiyoda-ku, Tokyo 101-8457, Japan), and John Pruitt (Microsoft Corp., Redmond, WA 98052)

The present study investigated brain plasticity in Japanese adults learning the English /1-r/ distinction. Both behavioral and brain (MEG) measures were used. Training was designed to mimic the listening experience of infants who are exposed to the exaggerated acoustic events contained in infant-directed speech ("motherese"). A multimedia software program incorporated major features conducive to perceptual learning. The program consisted of 12 1-h training sessions. Pre- and post-training measures were collected from nine subjects including two controls. The MEG experiments examined training effects in preattentive discrimination versus categorization conditions. An average improvement of $21.7 \%$ was obtained behaviorally. The training effect was significant for all factors of speaker, vowel, and syllabic context. Perceptual learning generalized to three untrained voices. No effects were observed in the two control subjects. Consistent with the behavioral results, the MEG data showed significantly improved mismatch responses in the left hemisphere. Both behavioral and brain data indicated that the learned phonetic boundary in Japanese subjects did not match that of the American listeners. This pattern of results suggests the existence of substantial neural plasticity in adulthood as well as a potential limit. [Work supported by NIH, HFSP, and the Talaris Research Institute.]

2pSC17. Effects of short-term exposure to a foreign language on discrimination of a non-native phonetic contrast: Convergent evidence from brain and behavioral tests. Patricia K. Kuhl, FengMing Tsao, Huei-Mei Liu, Sharon Corina, Erica Stevens, Tobey Nelson, Jessica Pruitt, and Denise Padden (Ctr. for Mind, Brain, and Learning, Univ. of Washington, Seattle, WA 98195)

Studies in our laboratory demonstrate that between 6 and 12 months of age infants show a significant increase in the ability to discriminate nativelanguage phonetic contrasts and a decline in foreign-language discrimination. The increase in performance on native-language contrasts suggests a process of active learning rather than maintenance. In the present experiment, we tested whether the learning process infants engage in during the period between 6 and 12 months extends to a foreign language the infants had not previously heard. American infants at 9 months of age participated in a 12-session language play group in which they heard a native speaker of Mandarin Chinese read, play, and talk to them. A control group was exposed to American English using the same books and toys. Both groups were subsequently tested on a Mandarin Chinese contrast using both behavioral and brain measures. The results demonstrate that infants exposed to Mandarin over a 4-week period show significant discrimination of the Mandarin contrast measured both behaviorally and with event-related potentials (ERP). The data, demonstrating that short-term exposure to language results in significant increases in performance, suggest a powerful learning process in language acquisition. [Research supported by NIH, HFSP, and the Talaris Research Institute.]
2pSC18. Developmental changes in the discrimination of Hindi dental-retroflex consonants by American-English children between childhood and puberty. Jesica C. Pruitt (Ctr. for Mind, Brain, and Learning \& Speech and Hearing Sci., Univ. of Washington, Seattle, WA), John S. Pruitt (Microsoft Corp., Redmond, WA), Tobey L. Nelson, and Patricia K. Kuhl (Ctr. for Mind, Brain, and Learning \& Speech and Hearing Sci., Univ. of Washington, Seattle, WA)

There are many studies comparing adult and infant perception of nonnative speech sounds, with results typically showing that infants outperform adults on non-native phonetic discrimination. However, there is a lack of research that investigates the developmental change in native and non-native speech perception, particularly from early childhood to puberty, when plasticity for learning a new language appears to decline. In an attempt to better understand the effects of language experience on native and non-native speech perception during this period, the current study examined 6 to 15-year-old American-English children's ability to discriminate Hindi dental-retroflex consonants. Discrimination data were obtained using four phonetically different consonant-vowel contrasts that are phonemic in Hindi but not in English. Discrimination data were also obtained using a native-language contrast ( $\mathrm{AE} / \mathrm{r} / \mathrm{vs} / \mathrm{l} /)$. For comparison, American-English and Hindi adults were tested. A newly developed computer-based testing method was used with all subject groups to present the various contrasts in an engaging and efficient manner. Findings suggest that there are interesting developmental trends in the age range of 6-15 years, and that the computer-based testing method will provide a valuable tool for assessing speech perception across a wide age range. [Work supported by NICHD and Talaris Research Institute.]

$2 \mathrm{pSC} 19$. Word segmentation in a foreign language: Further evidence for crosslinguistic strategies. Barbara Hoehle, Dorothee Giesecke (Dept. of Linguist., Univ. of Potsdam, P.O. Box 6015 53, 14415 Potsdam, Germany), and Peter W. Jusczyk (Dept. of Psych., Ames Hall, Johns Hopkins Univ., Baltimore, MD 21218-2686)

We report further evidence that early word segmentation is triggered by rhythmic properties of the language. Our study used the head turn preference paradigm to compare the skills of English and German 9-month-olds to extract bisyllabic trochaic German words from German text passages. Both English and German are stress-timed languages and have trochaic feet as their dominant stress pattern in bisyllabic words. Consequently, no differences in the segmentation strategies used by infants from these two language groups were expected. In line with our predictions, even though the stimulus material contained phonemes that do not belong to the English phoneme inventory and violations of English phonotactics, English-learners were as successful in detecting the words as German infants. These findings replicate those of Houston et al. (2000) with Dutch and English. Thus, our findings provide further evidence that children can exploit processing strategies developed in their own language to process another language. Furthermore, the data suggest that, at least initially, word segmentation in infancy relies on acoustical information in a pure bottom-up fashion without the support of existing knowledge about the language to be processed.

2pSC20. Eye movements and the activation of native words in non-native spoken-word recognition. Andrea Weber (MPI f. Psycholing., Wundtlaan 1, 6525 XD Nijmegen, The Netherlands and Speech and Hearing Dept., Grad. Ctr., CUNY, 365 5th Ave., New York, NY 10016-4309)

Using the eye-tracking paradigm, this study explored the recognition of non-native spoken words. In particular, it focused on whether listeners activate native candidate words during the recognition of non-native words. Dutch participants followed spoken instructions in English to click on pictures using a computer mouse, while their eye movements were monitored. A target picture (e.g., the picture of a desk) was presented along with distractor pictures. The Dutch name of a distractor picture was either phonologically related to the English name of the target picture 
(e.g., English target desk /dEsk/ and Dutch competitor deksel, "lid," /dEks@1/) or it was phonologically unrelated (e.g., bloem, "flower" or schommel, "swing"). Eye movements to a picture were interpreted as evidence for activation of the lexical representation associated with this picture. As the English target word unfolded over time, participants fixated distractor pictures with phonologically related Dutch names more than distractor pictures with phonologically unrelated names. The results demonstrate that listeners consider candidate words of the native language for recognition of a non-native word. Listeners are not able to deactivate the native mental lexicon even when they are in a monolingual non-native situation where the native language is irrelevant and possibly even misleading. [Work supported by MPI for Psycholinguistics.]

TUESDAY AFTERNOON, 4 DECEMBER 2001

ROOM 304, 12:55 TO 6:00 P.M.

\title{
Session 2pUW
}

\section{Underwater Acoustics and Archives and History: How Did We Get Here? Insights into the History of Underwater Acoustics}

\author{
Ralph R. Goodman, Chair \\ Applied Research Laboratories, Pennsylvania State University, P.O. Box 30, State College, Pennsylvania 16804-0030 \\ Chair's Introduction-12:55 \\ Invited Papers
}

1:00

2pUW1. Project Artemis-A retrospective view. Alan Berman (Unit A-3, 2300 W. Alameda St., Santa Fe, NM 87501)

Approximately 45 years ago, the Underwater Acoustics Community of the U.S. undertook a massive research program called Project Artemis. The objective of this endeavor was to determine what were the necessary capabilities needed to build an active mono-static sonar that was capable of detecting a submerged submarine at ranges of about $1000 \mathrm{~km}$. Although no operational capability ever resulted from this effort, the research undertaken by this project identified the limitations in existing technology and in the community's knowledge of underwater water acoustics that precluded achievement of the original objective. In the area of technology, the most obvious limitation was the limited speed of available computers that forced the use of cumbersome analog devices for signal processing and for beam steering. Techniques for controlling the location of the source dynamically and for the construction and for the operation of large-scale acoustic high-powered phased arrays were developed successfully. Unresolved issues that formed the basis for future research efforts related to signal coherence time, low-frequency acoustic cross sections of submarines, the development of reliable computation models for propagation loss, low-frequency acoustic absorption processes in ocean, and the development of algorithms that accounted for source Doppler. Project Artemis terminated in the mid-1960s.

\section{$1: 25$}

2pUW2. The ancient and selective history of the development of high frequency, short range, high resolution active sonars. Chester M. McKinney (Appl. Res. Labs., Univ. of Texas, P.O. Box 8029, Austin, TX 78713-8029)

This is a brief history of the development of a broad class of active sonars which generally have most or all of these characteristics: relatively high operating frequency $(35-1500 \mathrm{kHz})$, short range (a few meters to $1.0 \mathrm{~km})$, high range resolution $(1.0 \mathrm{~m}$ to a few $\mathrm{cm}$ ), and high cross range resolution (a few degrees to $0.1^{\circ}$ ). Emphasis is on such sonars developed by several navies to find small objects such as naval seamines although there are other important civilian applications. Beginning with the experimental work of Paul Langevin (France) in 1916-18, the development of three generic types of sonars is traced: ahead searching, multibeam, electronic scanning sonars; continuous transmission frequency modulated sonars; and side-scanning sonars. Other types such as small boat sonars, sonars for manned and unmanned small submersibles, and 3D or camera type sonars are covered briefly. Basic research to support the development of all these sonars, primarily target strength and echo structure and bottom reverberation, is discussed only briefly as are other topics such as techniques for high cross-range resolution, target classification clues, and uses for wide bandwidth. Sonars that have proven to be historically significant are identified. The period covered is primarily 1916-1980.

\section{$1: 50$}

2pUW3. Motivating the underwater acoustics community: 50s and 60s version. Fred N. Spiess (Marine Physical Lab., Scripps Inst. of Oceanogr., UCSD 0205, 9500 Gilman Dr., La Jolla, CA 92093-0205, fspiess@ucsd.edu)

In the decades immediately following the end of WWII our Navy was the only significant U.S. sponsor of underwater acoustics R\&D. There were close, collegial, interactions among the academic and Navy laboratory researchers as well as between those groups and the operation-oriented Navy community. There was direct contact between the operating forces and the working levels in the 
research organizations, exemplified by ComSubPac providing submarine services for research directly to the Navy Electronics Laboratory and the Marine Physical Laboratory. There was the ONR Deep Water Propagation Committee, where meetings started with a round table discussion of on-going research results before moving on to devising programs optimizing use of the research groups in the several academic and Navy laboratories. There was the Undersea Warfare R\&D Planning Council in which senior officers from CNO met and shared their concerns with the directors of the Navy and academic laboratories, including critical review of the Navy's undersea warfare $R \& D$ budgets. This paper will explore a range of similar examples and show how they contributed to maintaining a high level of enthusiasm, collegiality and inventiveness resulting in major improvements in Navy sonar capabilities yesterday and providing perspectives for tomorrow.

2pUW4. Towed array history, 1917-2001. Stanley G. Lemon (Cheasapeake Science Corp., 1127B Benfield Blvd., Millersville, MD 21108)

Towed array research and developments over 84 years is examined from the inventions of Dr. Harvey C. Hayes during World War I to multiple arrays towed by oil exploration ships of today. A time line of the effort over the 80 years is presented with a discussion of the driving technical and military reasons for system evolutions. Military surface ship towed arrays from Hayes electric eel to the AN/SQR-14, SURTASS, ETAS, and APL/Johns Hopkins dual towed arrays are reviewed. Submarine array programs are described from the early 1960s through today. A prediction of future towed array developments is presented.

2pUW5. Oceanic ambient noise. William M. Carey (Dept. of Aerosp. and Mech. Eng., Boston Univ., 110 Cummington St., Boston, MA 02215)

Ambient Noise (AN) research during World War II was facilitated by the availability of calibrated instruments and motivated by the necessity to understand AN levels in coastal waters. The state-of-the-art was reviewed by Knudsen in 1948 and later by Pryce and Urick in 1954. After the war, AN research waned until the classic paper by Wenz (1962) marked its renaissance. By 1976, a vast literature existed on measurements, theory and computational methods, and in the 1980s AN was the second largest area of underwater acoustics. Initially, emphasis was on the omni-directional properties of the field and later on vertical/horizontal directionality and the shipping-component statistical characteristics. Measurement arrays characterized the statistics of the AN, its persistent directionality, and benchmarked attempts to predict the AN-levels. Interest in AN sources re-emerged around 1985 with the identification oceanic bubble ensembles as a major contributor to deep and shallow water noise fields. This overview discusses fundamental AN sources, measurements, and the physics of the air-sea interaction boundary layer. Bubble, spray, splash, and rain noise are examined and ranked in order of importance. The production, absorption, and transmission of sound by bubble clouds and layers produced by breaking waves are examined. [Work sponsored by ONR.]

3:05-3:20 Break

2pUW6. Advances in high-frequency underwater acoustics. Edward G. Liszka (Office of Naval Res., 800 N. Quincy St., Ballston Towers One, Ste. 907, Arlington, VA 22217-5660)

Significant progress has been made over the past 3 decades in understanding the acoustic phenomena that affect the operation of high-frequency (i.e., $10 \mathrm{kHz}-60 \mathrm{kHz}$ ) sonars utilized by systems in the undersea environment such as weapons and autonomous underwater vehicles. The specific underwater acoustic research interests have somewhat paralleled and been motivated by the technology developments for these applications. In the late 1960s and early 1970s, experiments and model development focused on understanding the processes of signal propagation, scattering from the ocean medium (reverberation), and target reflection to predict signal levels for energy detection systems. Recently, systems have evolved to exploit more advancd signal processing concepts that have established a new set of scientific issues. Accordingly, underwater acoustics research has been focused on time/frequency/spatial distribution of energy, temporal, and spatial coherence of signals and background, and a broader scope of complex environments. The increased understanding has been captured in models representing the physical phenomena and rather detailed simulations of the underwater acoustic picture. This paper will review some of the key results in high-frequency underwater acoustics research that have contributed to the current state of knowledge and an indication of the direction for future research. 
2pUW7. From matched filter to matched field tracking. Homer Bucker (SPAWARSYSCEN, 53560 Hull St., San Diego, CA 92152, bucker@nosc.mil)

An old example of use of a matched filter in underwater sound is the quadrature detector. The signal from hydrophone \# $j$ is split, and one channel is matched with a cosine wave and the other with a sine wave. These channels are assembled as the real and imaginary parts of the analytic signal $F_{j}$. The next application of a matched filter occurs in plane wave beam forming where the signals $\left\{F_{j}\right\}$ of an array are matched with signals $\left\{G_{j}(\varphi)\right\}$, which are expected if the incident signal is a plane wave with direction $\varphi$. The problem is expanded when a more precise form of $\left\{G_{j}\right\}$ is used. In general, $\{G\}$ will be a function of the location of the source. Therefore, in principle, the location of a sound source can be found by finding the coordinates of the best match between the $F$ 's and the $G$ 's (matched field processing). Several methods will be described showing how to do this in an efficient manner. Finally, there is further gain to be had by including many time values in matching the track of the acoustic source (matched field tracking). Examples will be shown illustrating results from sea tests.

4:10

2pUW8. Chemical sound absorption in the ocean. Frederick H. Fisher (Marine Physical Lab., Scripps Inst. of Oceanogr., UCSD, La Jolla, CA 92093-0701, fhf@mpl.ucsd.edu)

Two salts cause significant sound absorption in the ocean, small concentrations of magnesium sulfate ion pairs and boric acid. Below $100 \mathrm{kHz}, \mathrm{MgSO} 4$ produces sound absorption $\sim 30$ times greater than that of pure water. For boric acid below $1 \mathrm{kHz}$, the excess absorption in the Atlantic over pure water is $\sim 300$ times and in the Pacific $\sim 150$ times, the difference being lower $p \mathrm{H}$ at the sound axis in the Pacific than in the Atlantic (7.7 vs 8), the borate concentration being the same. For MgSO4, a four state dissociation model of Eigen and Tamm provides an explanation of sound absorption at 1 ATM from $10 \mathrm{kHz}$ to $300 \mathrm{MHz}$. Remarkably, at 1000 ATM, this same model accounts for the observed large decrease in sound absorption $(-66 \%)$ and the small increase in electrical conductance $(+10 \%)$. For boric acid, Simmons explained absorption in terms of a three state ion pair model involving a configurational change of the $\mathrm{B}(\mathrm{OH}) 4$ ion from a tetrahedral to a planar trigonal structure $\mathrm{B}(\mathrm{OH}) 3$ involving a large volume change of $36 \mathrm{~cm} / \mathrm{mole}$. The discovery, some history and the current status of these once "anomalous" absorptions will be discussed. [Work supported by ONR and NSF.]

4:35

2pUW9. Fifty years of measuring, modeling, and sometimes understanding the physics of sound in the sea. Helen M. Walkinshaw (Box 72, Peapack, NJ 07977, hmckayw@juno.com)

By the end of the 1940s naval experience in World War II and progress in undersea physics and oceanogoraphy had brought about recognition of low frequency as a means of passive detection. The discovery of the deep sound channel was published in 1948, defining its role in long range propagation. In January 1950, the National Research Council's Committee on Undersea Warfare recommended a study be made of these emerging concepts. What came to be known as the Hartwell Committee was formed. The Committee's report included specific recommendation of real-time narrow band analysis of radiated sounds and exploitation of low-frequency signals. In October, the Chief of Naval Research was directed "to proceed with arrangements with Bell Laboratories to institute a program of research and development in the field of low frequency." This directive, shared with naval laboratories and affiliated universities, initiated an intensive program of sea experiments that continued over four decades. The measurements were paralleled by computer codes developed to analyze and deconstruct data, and eventually model propagation and arrival structure in realistic, range-varying ways. The sequence of the discovery and validation of features that define the nature of undersea sound as now understood are outlined and discussed.

5:00-6:00

Panel Discussion 